\title{
Ultrasonic Non-Destructive Testing of Fibre Reinforced Composites
}

by

Matthew Thomson

\author{
A thesis \\ submitted to the Victoria University of Wellington \\ in fulfilment of the \\ requirements for the degree of \\ Master of Science \\ in Physics.
}

Victoria University of Wellington

2012 



\begin{abstract}
This thesis focuses on the application of high frequency ultrasound as a tool for performing non-destructive testing for pultruded fibre reinforced composite (FRC) rods. These composite rods are popular in the manufacturing, construction and electrical industries due to their chemical, electrical and strength properties. Such FRCs are manufactured on automated production lines that operate day and night. Non-destructive testing techniques are desired to quickly and accurately detect manufacturing flaws such as coating thickness irregularities and surface cracks. Layers and cracks can present as large changes in acoustic impedance and will strongly reflect ultrasonic waves. Combined with their low cost, east of use and absense of potentially harmful radiation, ultrasound has proven popular worldwide for Non-Destructive Testing.
\end{abstract}

Finite Element Analysis (FEA) was employed to investigate the propagation of ultrasonic waves through layers of material to simulate a thickness measurement and the ability of ultrasound to measure thicknesses was proven. Experimental work was conducted on two fibre reinforced composite samples with varying thickness coatings of plastic and paint. The thickness was measured accurately using immersion transducers at $50 \mathrm{MHz}$ and a resolution of $20 \mu \mathrm{m}$ was attained through the use of matched filtering techniques.

Surface acoustic waves, particularly Rayleigh waves were investigated using FEA techniques so that the generation, scattering and detection of such waves was understood. This lead to the development of methods for detecting surface cracks in glass using Rayleigh waves and these methods 
were successfully used in experimental work. Wave propagation in fibre reinforced composites was modelled and experimentally investigated with the results confirming theoretical expectations. Finally a Rayleigh wave was launched onto a fibre reinforced composite sample however the amount of energy leakage into the water was so great, due to the acoustic impedance of water, the detection of the wave was prevented. The conclusion reached was that an immersion setup was not appropriate for launching a travelling Rayleigh wave. 


\section{Acknowledgements}

First and foremost I would like to thank my two supervisors Mr Paul Harris and Dr Gideon Gouws. I am greatful that Paul took me on as a Masters student and introduced me to the fascinating world of ultrasound. I've learnt a huge amount from Paul, not just about wave propagation and the technical side of research but on all other aspects of life. I thank Gideon for his continued enthusiasm and helpful guidance throughout the course of my studies, not just during this project but also during my undergraduacy.

Thanks to Dr Andrew Dawson who encouraged me to get involved with the ultrasound program and has been of great assistance throughout the project. I must also thank Dr Diana Siew for the funding of the project and the rest of the EAP/MDT/IMD group at Industrial Research Ltd for all their help at various stages. Particularly Freddy Lecarpentier for his help with cutting, polishing and imaging samples in the clean room and Alan Wright for the amazing photographs. Peter Bates and Marc Mulholland from the IRL Workshop continue to amaze me with their ability to find simple solutions to the many intricate problems I bring them.

Thanks to all the staff at VUW that taught me at some stage over the years and David Flynn for his expertise and willingness to help me with my SEM images at a moment's notice. To the Team Science/Engineering crew, thank you for the camaraderie during this learning journey, it's been great. 
I would like to thank my extended family and friends for all their support and encouragement. Finally I would like to heartily thank my parents for their interest, support and for putting a roof over my head for all these years. 


\section{Contents}

1 Introduction $\quad 1$

1.1 Thesis Outline ..................... 5

2 Theoretical Background $\quad 7$

2.1 Acoustic Wave Propagation ... . . . . . . . . . 7

2.1.1 Bulk Acoustic Waves . . . . . . . . . . . . 8

2.1.2 Surface Acoustic Waves ............. . . 9

2.2 Acoustic Impedance . . . . . . . . . . . . . . . . 14

2.3 Reflection and Transmission of Ultrasound . . . . . . . . . . 15

2.3.1 Normal Incidence . . . . . . . . . . . . . . . . . . 15

2.3.2 Oblique Incidence . . . . . . . . . . . . . 17

2.4 Attenuation . . . . . . . . . . . . . . 25

2.4 Absorption ................... 25

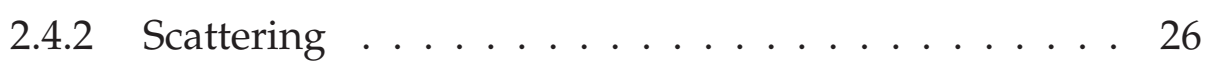

3 Experimental Setup $\quad 29$

3.1 Transducers . . . . . . . . . . . . . . . . . . . 29

3.1.1 Construction of Piezoelectric Transducers . . . . . . . 30

3.1.2 Transducer Properties . . . . . . . . . . . . . 32

3.2 Ultrasonic Techniques . . . . . . . . . . . . . . . . . . 42

3.2.1 Pulse-Echo Measurements . . . . . . . . . . . . . . . 42

3.2.2 Pitch-Catch Measurements . . . . . . . . . . . . . 44 
3.2.3 Simultaneous Pulse-Echo and Pitch-Catch Measurements . . . . . . . . . . . . . . 45

3.3 Electronics . . . . . . . . . . . . . . . . . 47

3.3.1 Power Amplifier . . . . . . . . . . . . . . . 48

3.3 .2 Switch . . . . . . . . . . . . . . . . 49

3.3.3 Variable Gain Amplifier . . . . . . . . . . . . . . . . . 49

3.3.4 Time Gain Compensation Variable Gain Amplifier . . 50

3.4 Signal Processing . . . . . . . . . . . . . . . 51

3.4.1 Matched Filtering . . . . . . . . . . . 51

4 Coating Thickness Measurements $\quad 55$

4.1 Sheathed $\operatorname{Rod} \ldots \ldots$. . . . . . . . . . . . 55

4.1.1 Sheath Thickness Model . . . . . . . . . . . . . 57

4.1.2 Finite Element Analysis . . . . . . . . . . . . . . 59

4.1 .3 Experimental ................ 65

4.2 Painted Rod . . . . . . . . . . . . . . . . 71

4.3 Conclusion . . . . . . . . . . . . . . 77

5 Surface Waves and Crack Detection $\quad 79$

5.1 Glass . . . . . . . . . . . . . . . . . . . 81

5.1.1 Experimental Velocity Measurements . . . . . . . 81

5.1 .2 Finite Element Analysis . . . . . . . . . . . . . . 81

5.2 Circular Cross Section Glass Rod . . . . . . . . . . . . . 90

5.3 Crack Detection . . . . . . . . . . . . . . . . . . . . 94

5.4 Fibre Reinforced Composite Rods . . . . . . . . . . . . . . . . 101

5.4.1 FEA of Wave Propagation . . . . . . . . . . . . 101

5.4.2 Measurement of Acoustic Properties . . . . . . . . . 106

5.4.3 Experimental Measurement of Rayleigh Waves . . . . 108

5.5 Conclusion . . . . . . . . . . . . . . . . . . 118

6 Summary and Conclusion $\quad 121$ 


\section{List of Figures}

1.1 Schematic of the pultrusion process. . . . . . . . . 2

2.1 Longitudinal wave. . . . . . . . . . . . . . . 8

2.2 Shear wave. . . . . . . . . . . . . . . 9

2.3 Rayleigh wave. . . . . . . . . . . . . . . . . 11

2.4 Leaky Rayleigh wave. . . . . . . . . . . . . . . . . . 12

2.5 Love wave. . . . . . . . . . . . . . . . . . . . . . . 14

2.6 Reflection and transmission of pressure wave normally incident at a boundary. . . . . . . . . . . . . 17

2.7 Reflection and transmission of pressure wave normally obliquely at a boundary. . . . . . . . . . . . . 18

2.8 Angular variation of the reflection coefficient of aluminium immersed in water. . . . . . . . . . . . . . . 23

2.9 Schoch displacement. . . . . . . . . . . . . . . . . . . 24

2.10 Absorption in water for the $\mathrm{MHz}$ range. . . . . . . . . . . 26

2.11 Ultrasonic scattering from a sphere. . . . . . . . . . . 28

3.1 Construction and beam properties of an ultrasonic transducer. 33

3.2 The lateral and axial resolution of the three transducers [1]. The axial resolution depends only on the frequency of the wave hence is independent of the individual transducer. . . 34

3.3 Results of shock testing of the three Olympus Panametrics transducers used in this research. . . . . . . . . . . . . . 37 
3.4 Measured response of transducers with flat glass reflector at focal length. The excitation pulse was a Hann windowed 5 cycle sinusoid (inset). The centre frequency was varied and the peak voltage recorded for each frequency. . . . . . . . . 38

3.5 Magnitude and Phase [2] of Olympus Panametrics V390 50MHz Immersion Transducer. . . . . . . . . . . . . . . . . . . . 40

3.6 Magnitude and Phase of Olympus Panametrics V372 20MHz Immersion Transducer. . . . . . . . . . . . . . . . . . . 40

3.7 Magnitude and Phase of Olympus Panametrics V327 10MHz Immersion Transducer. . . . . . . . . . . . . . . . . . 41

3.8 Ultrasonic pulse-echo technique. . . . . . . . . . . . . 43

3.9 Ultrasonic pitch-catch technique. . . . . . . . . . . . . . 44

3.10 Simultaneous ultrasonic pitch-catch and pulse-echo technique. . . . . . . . . . . . . . 46

3.11 Schematic of ultrasound system. . . . . . . . . . . . 48

3.12 Switching electronics. . . . . . . . . . . . . . . 50

3.13 Matched filter process. . . . . . . . . . . . . 53

4.1 Schematic of pultruded rod with longitudinal crack. . . . . . 56

4.2 Sheathed rod concentricity model. . . . . . . . . . . 58

4.3 Sheathed rod FEA thickness measurement. . . . . . . . . . 60

4.4 Blak pulse from PZFlex. . . . . . . . . . . . . . . 61

4.5 Sheathed rod FEA snapshots. . . . . . . . . . . . 63

4.6 Sheathed rod FEA pressure time history. . . . . . . . . . . . 64

4.7 Photographs of immersion setup. . . . . . . . . . . . 68

4.8 Experimental oscilloscope trace for sheathed rod. . . . . . . 69

4.9 Experimental sheath thickness measurement results. . . . . . 70

4.10 Experimental matched filter properties. . . . . . . . . . . 72

4.11 Process of matched filter and thickness measurement for painted rod. . . . . . . . . . . . . . 73

4.12 Accuracy and resoltion matched filter for painted rod. . . . . 75

4.13 Typical oscilloscope trace for painted thickness measurement. 76 
4.14 Repitition of paint thickness measurement. . . . . . . . . . 77

5.1 Schematic representation of FRC rod. . . . . . . . . . . . 80

5.2 FEA of critical angle Rayleigh wave generation in glass. . . . 82

5.3 FEA snapshots of leaky Rayleigh wave in glass. . . . . . . . 86

5.4 FEA verification of Rayleigh wave on glass. . . . . . . . . . . 87

5.5 Experimental configuration for leaky Rayleigh wave generation and detection. . . . . . . . . . . . . . 88

5.6 Experimental method to verify the existence of a leaky Rayleigh wave on a fluid loaded surface. . . . . . . . . . . . 89

5.7 Finite Element model to investigate propagation of a leaky Rayleigh wave around a curved surface . . . . . . . . . 90

5.8 Snapshots of a leaky Rayleigh wave propagating around a curved surface. . . . . . . . . . . . . . . . . . . 92

5.9 FEA velocity of Rayleigh wave propagating around a curved surface. . . . . . . . . . . . . . . 93

5.10 Simulation of leaky Rayleigh wave behaviour at a surface crack in glass. . . . . . . . . . . . . . . . . . . . . 98

5.11 Pulse-echo Measurement for crack detection on glass. . . . . 99

5.12 Experimental verification of Rayleigh wave on glass. . . . . 100

5.13 Micrographs of a cut and polished cross section of the fibre reinforced composite rod. . . . . . . . . . . . 103

5.14 SEM images of cut and polished cross section of fibre reinforced composite rod. . . . . . . . . . . . . . . . . . . . 104

5.15 FEA model of plane of isotropy in FRC rod. . . . . . . . . . . 105

5.16 FEA snapshots of FRC rod cross section. . . . . . . . . . . . 111

5.17 FEA snapshots of leaky Rayleigh wave on FRC. . . . . . . . 112

5.18 Experimental specular and non-specular reflections for FRC. 113

5.19 Specular reflection blocker. . . . . . . . . . . . . . . . . . 114

5.20 Experimental verification of Rayleigh wave in FRC. . . . . . 114

5.21 FEA simulation of leaky Rayleigh wave propagating along the surface of the FRC with low density water. . . . . . . . 117 


\section{Chapter 1}

\section{Introduction}

Fibre Reinforced Composites (FRC) are modern composite materials constructed by embedding glass- or carbon-fibres in an epoxy resin to increase directional strength. A popular method of manufacturing glass FRC products is the Pultrusion process, a portmanteau of 'pull' and 'extrusion' [3]. The pultrusion process is briefly described with reference to Fig. 1.1. Coils of small diameter $(\sim 20 \mu \mathrm{m})$ fibres are fed into alignment cards to ensure the fibres are parallel and regularly spaced. The fibres are pulled through a resin bath before a heated, shaped die cures the resin. The shape can vary from simple circles resulting in cylindrical rods, ' $\mathrm{L}$ ' shapes to complicated window frame sections. Once the resin is cured the pultruded composite is cut to length. The process is continuous and factories often operate 24 hours a day.

The result is a lightweight $\left(\rho \sim 2000 \mathrm{kgm}^{-3}\right.$ [4]) composite that is very strong, stiff and durable. These composite are resistant to rust/chemical corrosion hence are popular choices over steel as concrete reinforcing in marine or other harsh environments, such as mines. The use of glass and resin ensures electrical insulation $\left(R \sim 10^{11} \Omega\right)$ and electromagnetic transparency making them ideal for environments where low magnetic field variations are desired, for example concrete reinforcing for a hospital MRI 


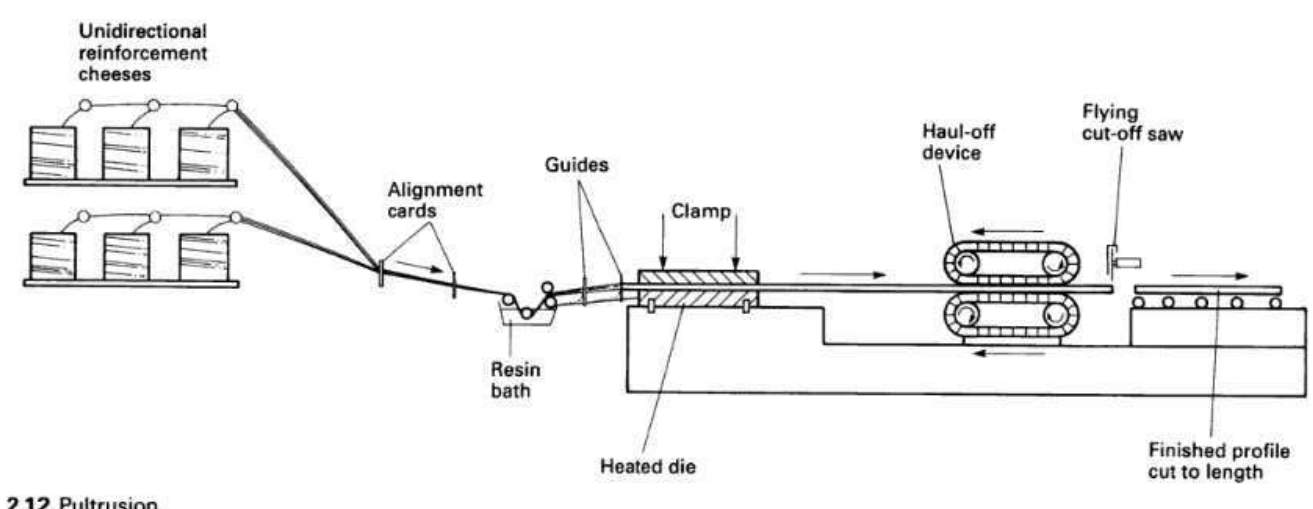

Figure 1.1: Schematic of the pultrusion process [3]. Fibres are aligned parallel and dipped in resin before shaping, curing and cutting.

room. Other applications include ladders, posts for electric fences, tent poles and sports equipment [3].

For applications with high exposure to UV or harsh environments, a protective coating is applied to the FRC rod. A common coating is a plastic sheath approximately $1 \mathrm{~mm}$ thick [5]. The sheath is attached by passing the FRC through an extruder. However the FRC may move out of alignment during the process, resulting in an inconsistent sheath thickness. The sheath thickness can be observed by eye once the sample is cut, however this is a destructive process. Another coating is paint, typically $100 \mu \mathrm{m}$ thick. The paint coating is applied after the product is cut to length. The FRC is placed in a holder that rotates the rod about the longitudinal axis while a paint sprayer moves along the length of the rod. This can result in an undesirable inconsistency of paint thickness. Such a thin paint thickness is extremely difficult to measure by eye and like the sheathed option would be destructive in nature.

Other manufacturing flaws known to occur in pultruded FRC rods include surface cracks. These cracks can be approximately one tenth of a millime- 
ter wide and one millimetre deep. The direction of the cracks is often longitudinal i.e. along the length of the sample. Although these surface cracks are small they can be potentially damaging to an FRC rod as they provide a location for larger cracks to develop. Large cracks can be detected by eye or by using one's finger nail. However since pultrusion production lines typically operate 24 hours a day it would be inefficient to have workers watch the sample during manufacture.

The aim of this thesis was to investigate the application of high frequency ultrasound to typical problems in FRC production. Two particular problems were addressed, the first was the measurement of the coating thickness around an FRC rod. The second was the identification of surface cracks in FRC rods. For both situations the aim was not to develop a system for a production line, as the interfacing with equipment in a factory was beyond the scope of the work. Moreover a study was conducted into wave propagation and reflection/transmission phenomena, with particular interest into the accuracy of the sytstem and use of signal processing techniques with equipment readily available.

Thickness measurements are the fundamental ultrasonic non-destructive testing (NDT) measurement, recording simple time of flight (TOF) data $t$ and calculating a distance $d$ for a known velocity $v$ via $d=v t$. These measurements are simple as they can be performed in a pulse-echo setup requiring only one transducer. The use of longitudinal waves reduces complications involved with mode conversion and separation of the various wave types in time. For this coating thickness work, Finite Element Analysis (FEA) was used to investigate the transmission/reflection phenomena of ultrasonic waves at boundaries, such as those between the coating and FRC. Signal processing techniques were employed to improve the accuracy of the measurement. 
For the potential detection of surface cracks, ultrasonic surface waves, particularly Rayleigh waves, were chosen as they are restricted to the surface of a sample. Ultrasound will always partially reflect when an impedance difference is encountered, with the reflected energy propotional to the acoustic impedance difference at the interface. A crack, especially when filled with air, has a dramatically different impedance to that of the solid rod hence surface waves can reflect strongly. The guiding nature of Rayleigh waves makes them a popular choice for surface probes $[6,7,8]$. Popular techniques for launching Rayleigh waves on FRC samples include laser ultrasonics [9] or double wedge arrangements with contact transducers [10]. In the literature these techniques were used for determining engineering constants and for evaluating interlaminar bonding.

Immersion transducers were chosen for this work due to their practicality; a sample and a transducer can be simply placed in a water bath with the water ensuring uniform coupling to surfaces that are rough or not flat, such as FRC rods. Another advantage of water coupling is the low velocity relative to most solids, enabling various wavetypes to be generated in the solid via critical angle excitation [11]. Immersion transducers can have focussing lenses to increase the intensity of the ultrasonic pulse. The devices used in this work were relatively cheap, approximately $\$ 2,000$ NZD per unit and were incorporated into an existing ultrasound system with the necessary electronics and position stages available [2]. In contrast, laser systems are costly (approximately $\$ 50,000 \mathrm{NZD}$ ) as they require separate lasers and optics for the generation of ultrasound and interferometry.

As stated above, the aim of this work was not to develop a fully functioning system that could be placed on a production line. This work was rather aimed at investigating the propagation of surface waves, the behaviour of Rayleigh waves at cracks and the effect of the fibres as scatterers. The potential for using these waves as a tool for surface crack detection was 
assessed.

Throughout this work FEA was conducted using the software package PZFlex [12] from Wiedlinger Associates Inc. The FEA functioned by dividing a $2 \mathrm{D}$ (or 3D) model space into a finite number of elements, with corresponding nodes separating the elements, such that partial differential equations for strain and stress could be solved at each element. This allows fluids and solids to be modelled in a wide variety of user defined configurations. The software was designed to be run on desktop PCs and provides a helpful insight into ultrasonic wave propagation in a short time i.e. minutes or hours instead of days. The results of the FEA simulations can be movie files of the ultrasonic wave propagation and time histories of numerical quantities eg. pressure. From the movie files, snapshots were chosen which are presented in the following chapters to demonstrate the results of the simulations.

\subsection{Thesis Outline}

Chapter 2 provides an introductory background into the theory of ultrasonic wave propagation particularly focussing on the wave types used in this work. The concept of acoustic impedance is introduced which leads to the transmission and reflection of ultrasonic waves. The angular variation of reflection is discussed and provides an insight into the generation of the various wave types, particularly the non-specular reflections and beam shifting associated with leaky Rayleigh waves. Finally the mechanisms of attenuation are covered.

The equipment and methods employed and developed in this work are detailed in Chaper 3. Piezoelectricity is explained and the construction of ultrasonic transducers is presented. Methods of testing a transducer's electrical impedance and frequency response are detailed. Common non- 
destructive testing techniques are introduced with particular attention to those used in this work. The high frequency electronic circuits neccessary for accurate measurements are detailed and the signal processing techniques used for analysing recorded data are explained.

Chapter 4 details the FEA and experiments conducted to test the accuracy of an ultrasonic pulse echo measurement for determining the thickness of a coating on a fibre reinforced composite rod. A rod with a plastic sheath $\sim 1 \mathrm{~mm}$ thick was investigated and the resolution limits of the system were tested by measuring the paint thickness $(\sim 100 \mu \mathrm{m})$ of a painted rod using radar signal processing techniques.

An investigation into the propagation of surface acoustic waves, particularly Rayleigh waves, is presented in Chapter 5. FEA was used to model the propagation of a leaky Rayleigh wave and experiments to confirm the existence of Rayleigh waves were conducted leading to the development of techniques to detect surface cracks.

The work is concluded with a discussion of possible improvements or alternative methods in Chapter 6. 


\section{Chapter 2}

\section{Theoretical Background}

This chapter references popular texts $[11,13,14,15,16,17]$ and provides an introduction into the theory of ultrasonic waves and associated phenomena. Section 2.1 covers the wave types and corresponding motion of particles, with emphasis on those wave types relevant to this research. The concept of acoustic impedance is covered in Section 2.2, followed by reflection and transmission in Section 2.3. Finally, aspects of attenuation are presented in Section 2.4.

\subsection{Acoustic Wave Propagation}

Ultrasonic waves are acoustic waves with frequencies above the range of human hearing $(>20 \mathrm{kHz})$ and can be classified as either bulk, surface or plate/rod waves. Bulk acoustic waves (BAW) exist in materials that have dimension much greater than the wavelength. Surface acoustic waves (SAW) propagate along the surface of a semi-infinite medium, with particle displacement a few wavelengths deep. Plate and rod waves exist in materials where the material thickness is of the order of a wavelength. 


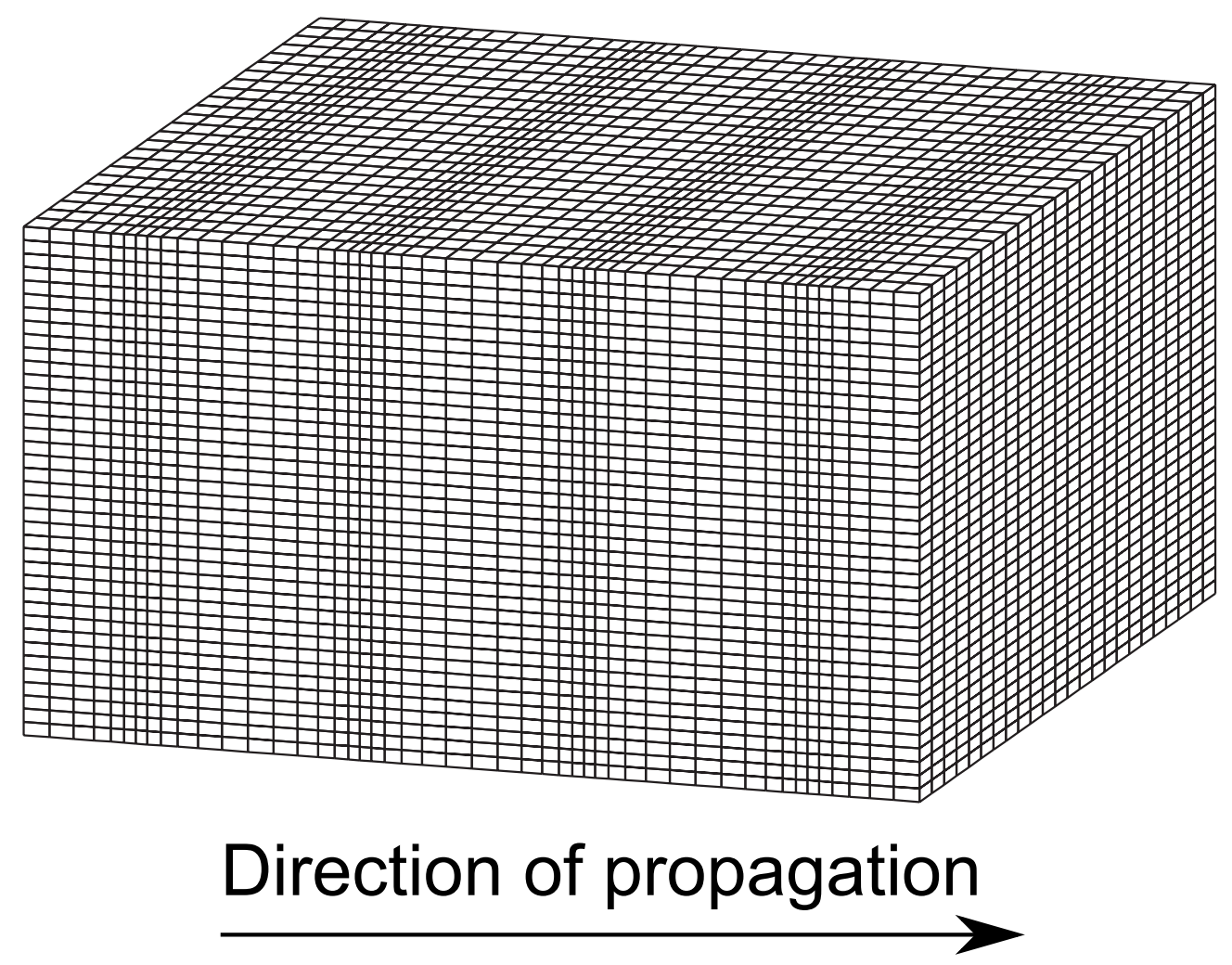

Figure 2.1: Grid deformation for a Longitudinal $(\mathrm{P})$ wave travelling left to right, after [11].

\subsubsection{Bulk Acoustic Waves}

Longitudinal, or $\mathrm{P}$, waves are elastic mechanical oscillations of particles about an equilibrium position, parallel and anti-parallel to the direction of wave propagation, seen in Fig. 2.1. P waves are the only waves that propagate through all three states of matter.

Shear, or S, waves involve a mechanical oscillation of the particles perpendicular to the direction of wave (energy) propagation, seen in Fig. 2.2. This perpendicular oscillation has an associated polarisation that can be classified as either shear vertical (SV) or shear horizontal (SH). SV waves are simple to excite via mode conversion, covered in Section 2.3.2, whereas 


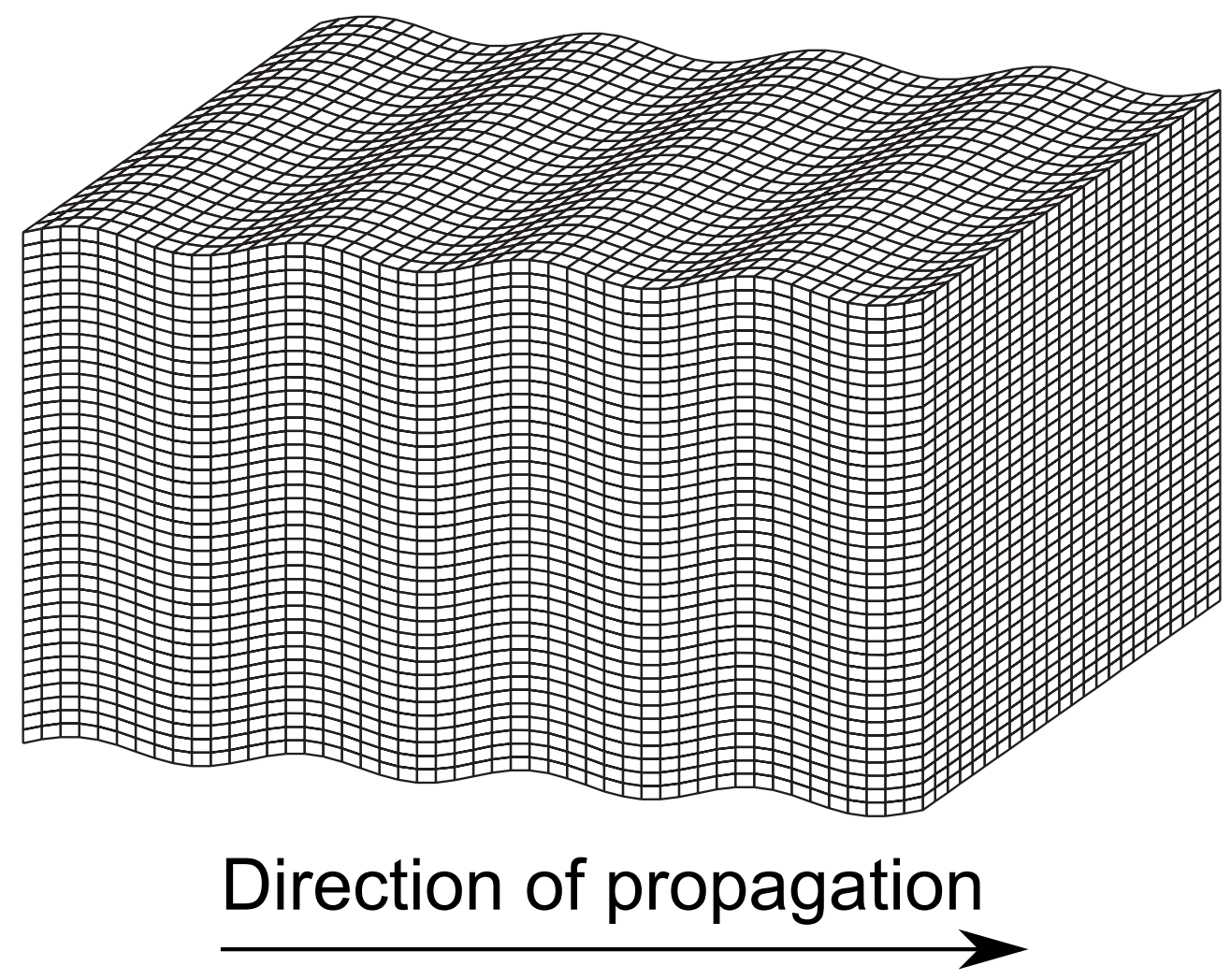

Figure 2.2: Grid deformation for a Shear (SV) wave travelling from left to right, after [11].

$\mathrm{SH}$ waves require a $\mathrm{SH}$ source $[11,14]$. Liquids and gases cannot support a static shear stress and correspondingly cannot support a shear wave hence shear waves are only found in solids. The velocity of a shear wave is approximately half that of a $\mathrm{P}$ wave in the same material [11].

\subsubsection{Surface Acoustic Waves}

The most common surface acoustic wave is the Rayleigh wave, first proposed by Lord Rayleigh in 1885 [18] as an explanation for the rolling motion of earthquakes. These waves propagate along, and are guided by, the surface of a semi-infinite solid. Rayleigh waves have particle displace- 
ments approximately one wavelength deep from the surface [11]. The motion of the particles in a Rayleigh wave is a coupling of longitudinal and shear (SV) waves, resulting in particles vibrating in an elliptical fashion, seen in Fig. 2.3. The vibration of particles at the surface is counterclockwise, however the motion changes to clockwise deeper below the surface. The Rayleigh equation relates the longitudinal, shear and Rayleigh velocities and is defined $[11,14]$

$$
\frac{V_{R}^{6}}{V_{S}^{6}}-8 \frac{V_{R}^{4}}{V_{S}^{4}}+V_{R}^{2}\left(\frac{24}{V_{S}^{2}}-\frac{16}{V_{L}^{2}}\right)-16\left(1-\frac{V_{S}^{2}}{V_{L}^{2}}\right)=0
$$

where $V_{R}$ is the Rayleigh velocity, $V_{S}$ is the shear velocity and $V_{L}$ is the longitudinal velocity. $V_{R}$ has a single real root that can be defined

$$
V_{R}=\frac{0.87+1.12 \sigma}{1+\sigma} V_{S}
$$

where $\sigma$ is Poisson's ratio, defined as the ratio of the expansion of a material in orthogonal directions $x$ and $y$, to the compression of the material in the orthogonal direction $z$. For the physically realisable range of Poisson's ratio, $0<\sigma<0.5$, the Rayleigh velocity is in the range of $0.87 V_{S}$ to $0.96 V_{S}[11,19]$. The Rayleigh velocity will always be slightly smaller than the shear velocity and approximately half the longitudinal velocity. Since the Rayleigh velocity is very close to that of a shear wave, the majority of energy is associated with the transverse component of oscillation, seen in Fig. 2.3, where the normal displacement (corresponding to shear) is greater than the tangential (corresponding to longitudinal).

If a surface is fluid loaded, such as when a sample is immersed in water, and a Rayleigh wave propagates along the surface of the sample, the fluid molecules near the surface will follow the elliptical motion of the surface. As the motion of the Rayleigh wave is predominantly transverse, the fluid molecules will move predominantly vertically, generating a longitudinal wave in the fluid. This longitudinal wave will propagate away from the surface at the Rayleigh critical angle, derived from Snell's law [11] 


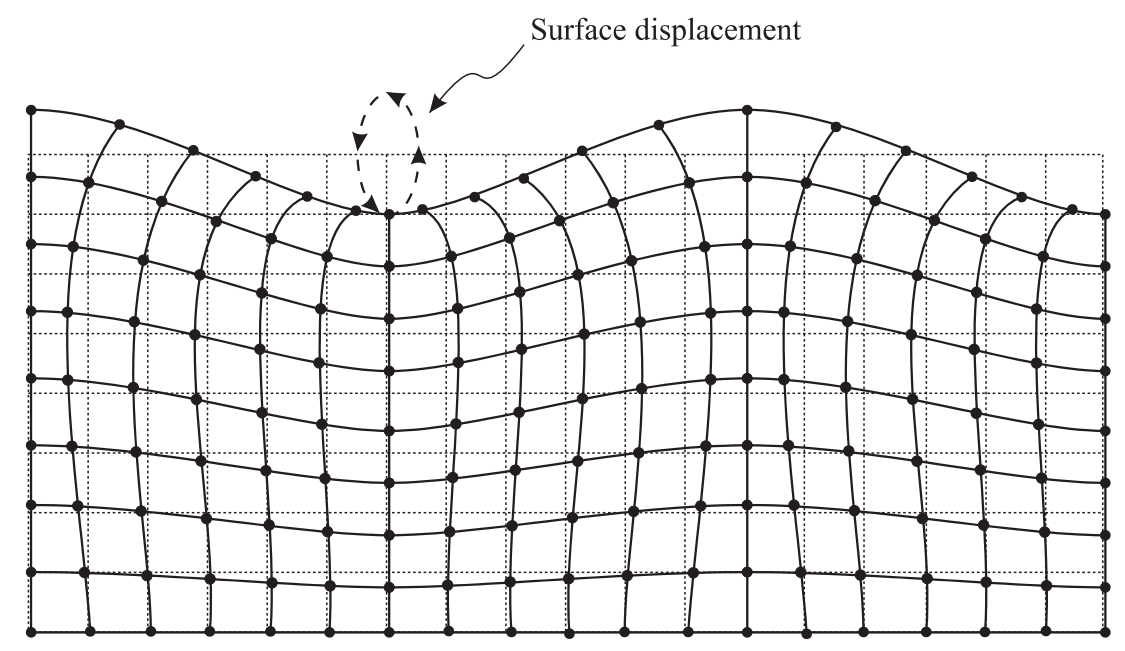

\section{Direction of propagation}

Figure 2.3: Grid deformation for a Rayleigh wave travelling left to right, after [11].

$$
\theta_{R}=\sin ^{-1} \frac{V_{F}}{V_{R}}
$$

which is measured relative to the surface normal and where $V_{F}$ is the longitudinal velocity of the acoustic wave in the fluid. Trigonometry requires $V_{F}$ to be less than the Rayleigh velocity. At the Rayleigh critical angle, the horizontal components of the longitudinal wave in the fluid and the Rayleigh wave are phase matched. This generation of a longitudinal wave requires energy, which is taken from the Rayleigh wave. The Rayleigh wave is deemed to be 'leaking' energy into the fluid and the Rayleigh wave is termed a leaky Rayleigh wave [11]. A finite element simulation of a leaky Rayleigh wave is seen in Fig. 2.4.

The amplitude of the leaky Rayleigh wave $A$ is defined 


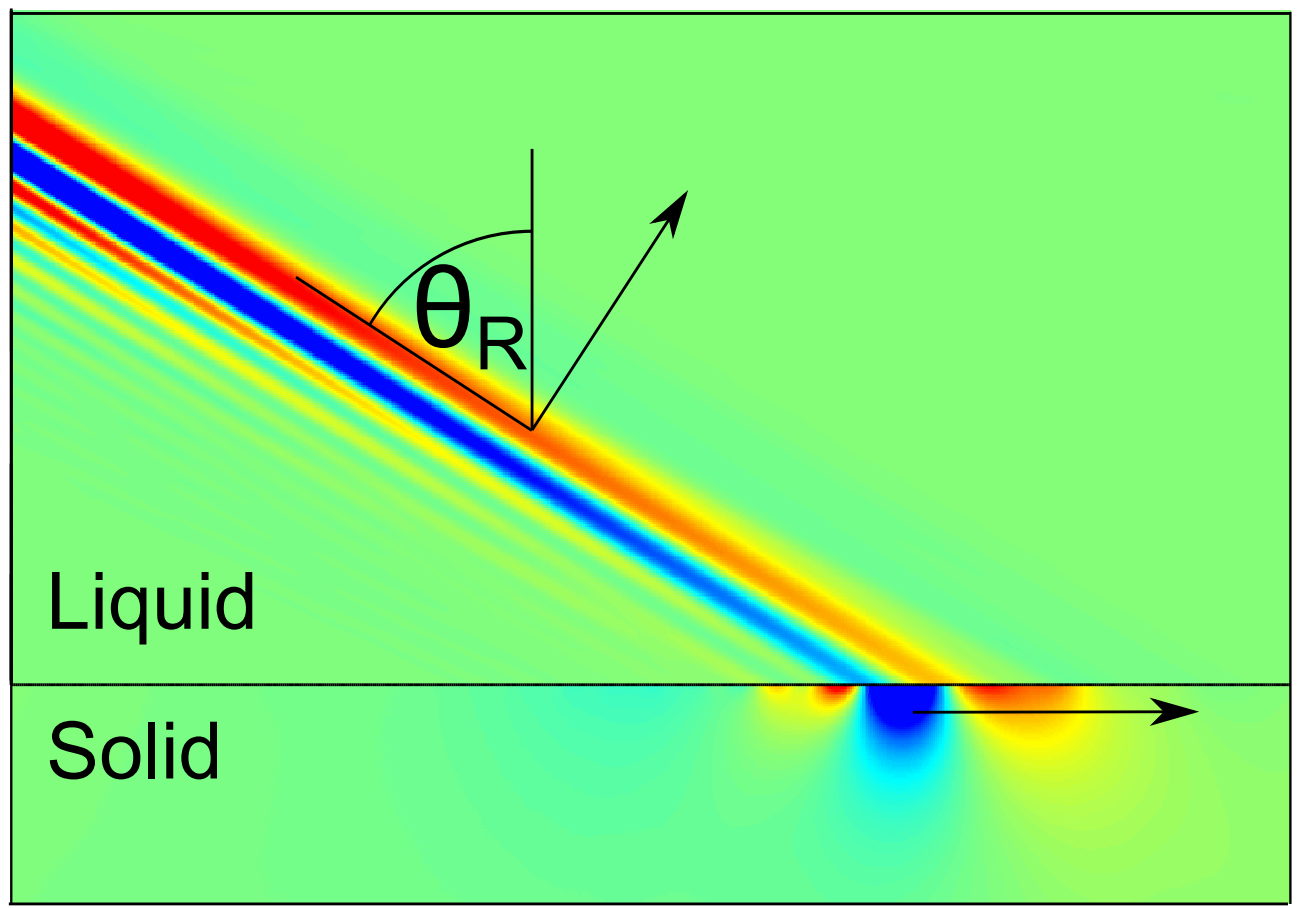

Figure 2.4: Finite Element Model of a leaky Rayleigh wave propagating along the surface of a solid immersed in a liquid. The leaked energy is propagating at the Rayleigh angle $\theta_{R}$ relative to the surface normal.

$$
A=A_{0} \exp (-\alpha z)
$$

where $A_{0}$ is the initial amplitude, $\alpha$ is the energy attenuation coefficient and $z$ is the distance propagated along the surface. The relationship is a decaying exponential because of the energy leakage and causes (2.2) to become complex. The energy attenuation coefficient $\alpha$ is defined [11]

$$
\alpha=\frac{\rho_{F} V_{F}}{\rho_{S} V_{R} \lambda}\left[\mathrm{cm}^{-1}\right]
$$

where $\rho_{F}$ and $\rho_{S}$ are the mass densities of the fluid and solid respectively and $\lambda$ is the wavelength. A characteristic distance of a leaky Rayleigh wave can be defined as the distance a Rayleigh wave travels along a sur- 
face before its amplitude is reduced by a factor $1 / e$. This distance is the inverse of the attenuation coefficient. For a Rayleigh wave on a glass surface $\left(V_{R}=3000 \mathrm{~ms}^{-1}\right.$ and $\left.\rho_{S}=3000 \mathrm{kgm}^{-3}\right)$ immersed in water $\left(V_{F}=1480 \mathrm{~ms}^{-1}\right.$ and $\rho_{F}=1000 \mathrm{kgm}^{-3}$ ), this characteristic distance is 6 wavelengths. After the leaky Rayleigh wave has travelled 3 times this characteristic distance its amplitude will have reduced to $(1 / e)^{3}=5 \%$ of its original, hence the range over which this Rayleigh wave will be easily detectable is limited to 18 wavelengths. For a $5 \mathrm{MHz}$ Rayleigh wave on the glass-water interface, this corresponds to approximately $11 \mathrm{~mm}$.

This leaking of energy works in reverse, where a longitudinal wave in the fluid impinging the surface at the Rayleigh critical angle will generate a Rayleigh wave on the surface. This is known as critical angle excitation [11] and is the method used in this work to generate leaky Rayleigh waves. Other methods for generating Rayleigh waves include interdigital transducers on piezoelectric substrates [14] or coupling shear wave transducers to a surface with oil [11].

Other surface acoustic waves exist, notably Love waves, which are similar to Rayleigh waves in that they propagate only at the surface of a material with particle displacement decaying rapidly with depth from the surface [11]. The difference between Love and Rayleigh waves is the wave motion, Rayleigh waves are coupled P and SV waves whilst Love waves are purely SH in nature but are restricted to the surface, seen in Fig. 2.5. This restriction is due to the velocity of a Love wave being less than the shear velocity. Of note is that the Love wave velocity is greater than that of a Rayleigh wave. Love waves are also present in earthquakes [20]. 


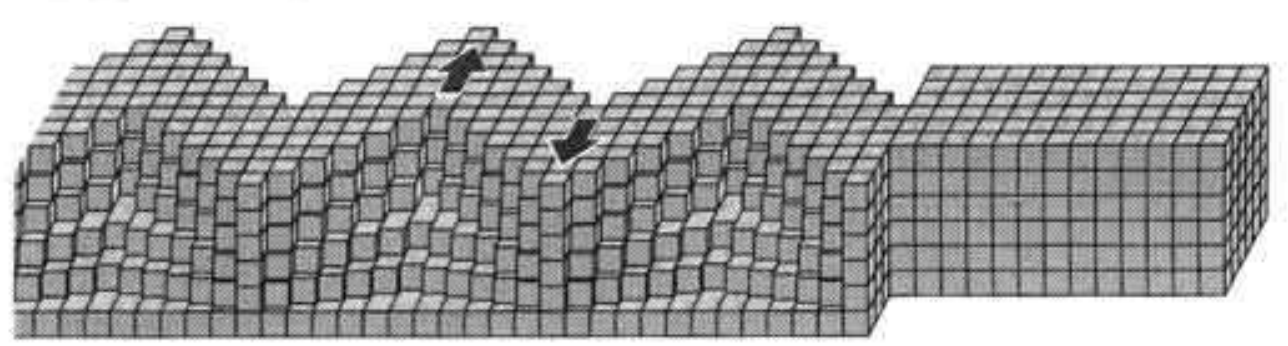

Love Wave

Figure 2.5: Love wave, with particle displacements perpendicular to direction of propagation approximately a wavelength deep from the surface, after [21].

\subsection{Acoustic Impedance}

The specific acoustic impedance $Z$ of an acoustic wave in a medium is defined [13]

$$
Z=\frac{p}{v} \quad\left[\frac{k g m}{s^{2} m^{2}} * \frac{s}{m}=\frac{k g}{m^{2} s}\right] .
$$

where $p$ is the acoustic pressure and $v$ is the particle velocity. The direction of propagation is included resulting in the impedance having either a positive or negative sign. This impedance is analogous to the electrical impedance defined by Ohm's law, with the pressure analogous to the voltage and the particle speed to the current. For plane waves the absolute value of $Z$ is the characteristic impedance, defined as

$$
|Z|=\rho V_{L} \quad\left[\frac{k g}{m^{3}} * \frac{m}{s}=\frac{k g}{m^{2} s}\right]
$$

where $\rho$ is the density of material and $V_{L}$ the longitudinal velocity. As the specific impedance is analogous to Ohm's law, the characteristic impedance is analogous to the electrical transmission line, where maximum transmission of energy occurs when characteristic impedances are matched. Where 
there is a characteristic impedance mismatch the transmission of energy is accompanied by a partial reflection of energy. The unit for impedance is defined in (2.6) and is commonly referred to as a Rayl, named after Lord Rayleigh. Common materials have impedances of the order of millions of Rayls, so MRayl are often used [11].

\subsection{Reflection and Transmission of Ultrasound}

Ultrasonic measurements involve waves propagating through a range of media, particularly fluids and solids, that have distinct boundaries. When a wave is normally incident on a boundary with an impedance mismatch, the wave will partially reflect and partially transmit. For oblique incidence, the transmitted wave will undergo refraction and mode conversion. The extent to which these phenomena occur is determined by the acoustic impedance mismatch, media type and angle of incidence. Plane waves are assumed hence there is the ability to use geometrical ray theory [13]. This section will describe the reflection and transmission phenomena for homogeneous fluids and solids at normal and oblique incidences.

\subsubsection{Normal Incidence}

The simplest case involves a plane wave propagating through a medium with characteristic impedance $Z_{1}$ that is in intimate contact with a second medium with impedance $Z_{2}$. The wave is normally incident on the boundary and undergoes partial reflection and transmission due to the acoustic impedance mismatch, with no mode conversion, as seen in Fig. 2.6. At the boundary the media remain in contact hence the boundary conditions are continuity of pressure and velocity [11]. The pressure reflection coefficient $R_{p}$ is defined as the fraction of pressure reflected from the boundary $p_{r}$ relative to the incident pressure $p_{i}$. The pressure reflection coefficient can 
be determined [13] as a function of the acoustic impedances

$$
R_{p}=\frac{Z_{2}-Z_{1}}{Z_{1}+Z_{2}}
$$

as can the pressure transmission coefficient $T_{p}$

$$
T_{p}=\frac{2 Z_{2}}{Z_{1}+Z_{2}}
$$

The acoustic intensity $I$ is defined [11]

$$
I=\frac{p^{2}}{2 Z}
$$

hence the acoustic intensity reflection and transmission coefficients, $I_{r}$ and $I_{t}$ respectively, are defined [11]

$$
\begin{aligned}
& \frac{I_{r}}{I_{i}}=\left|R_{p}\right|^{2} \\
& \frac{I_{t}}{I_{i}}=\frac{Z_{1}}{Z_{2}}\left|T_{p}\right|^{2}
\end{aligned}
$$

where $I_{i}$ is the incident intensity. This confirms conservation of energy

$$
I_{i}=I_{r}+I_{t}
$$

For the case of no impedance mismatch i.e. $Z_{1}=Z_{2}$ complete transmission will occur $\left(T_{p} \rightarrow 1\right.$ and $\left.R_{p} \rightarrow 0\right)$ as if there were no boundary. To contrast, when $Z_{2} \ll Z_{1}$, such as a water-air boundary with impedances of 1.5MRayls and 0.0004MRayls respectively, the reflection and transmission coefficients are $R_{p}=-0.99942$ and $T_{p}=0.00058$. The negative value of $R_{p}$ corresponds to a $\pi$ phase change of the reflected wave relative to the incident. 


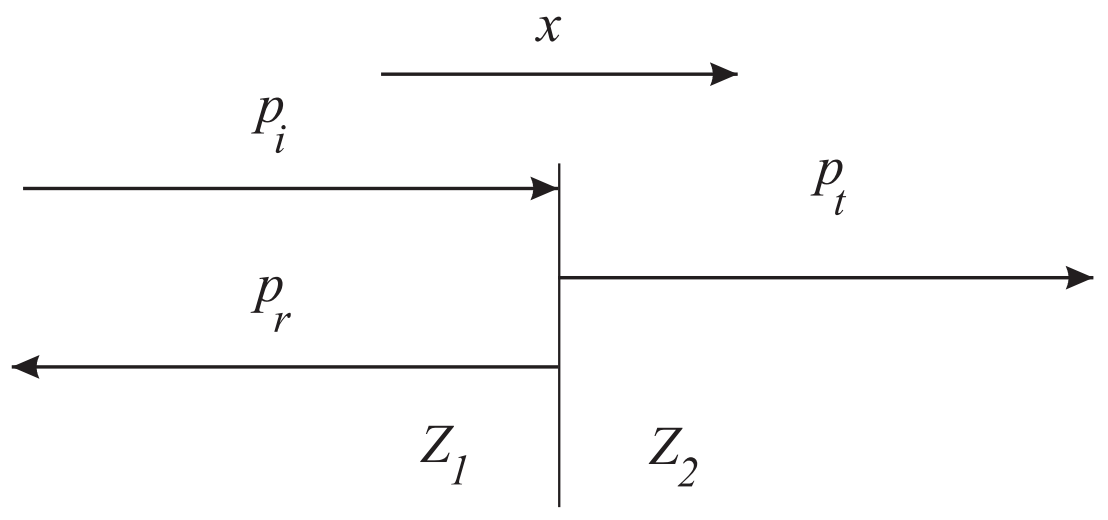

Figure 2.6: An interface between two media with an acoustic wave normally incident from the left having pressure $p_{i}$, the reflected wave with pressure $p_{r}$ and transmitted wave with pressure $p_{t}$. The media have characteristic impedances $Z_{1}$ and $Z_{2}$. Figure after [11].

\subsubsection{Oblique Incidence}

When an ultrasonic wave is incident at an oblique angle to a liquid-liquid boundary there will be a partial reflection and transmission that will depend on the angle of incidence. For a given angle of incidence $\theta_{i}$ the angle of reflection $\theta_{r}$ will equal the angle of incidence and the transmitted wave will refract at an angle $\theta_{L}$ defined by Snell's Law [11]

$$
\frac{\sin \theta_{i}}{V_{1}}=\frac{\sin \theta_{r}}{V_{1}}=\frac{\sin \theta_{L}}{V_{2}}
$$

where $V_{1}$ is the longitudinal velocity in liquid 1 and $V_{2}$ the longitudinal velocity in liquid 2. As with optics, critical angles exist such that total internal reflection will occur. Extending this to a liquid-solid interface seen in Figure 2.7, two waves are generated in the solid, a longitudinal wave (with velocity $V_{2 L}$ ) and a shear wave (with velocity $V_{2 S}$ ). This is mode conversion, where energy is converted between different wave modes. Again Snell's Law is obeyed and each wave type has a distinct angle of refraction 


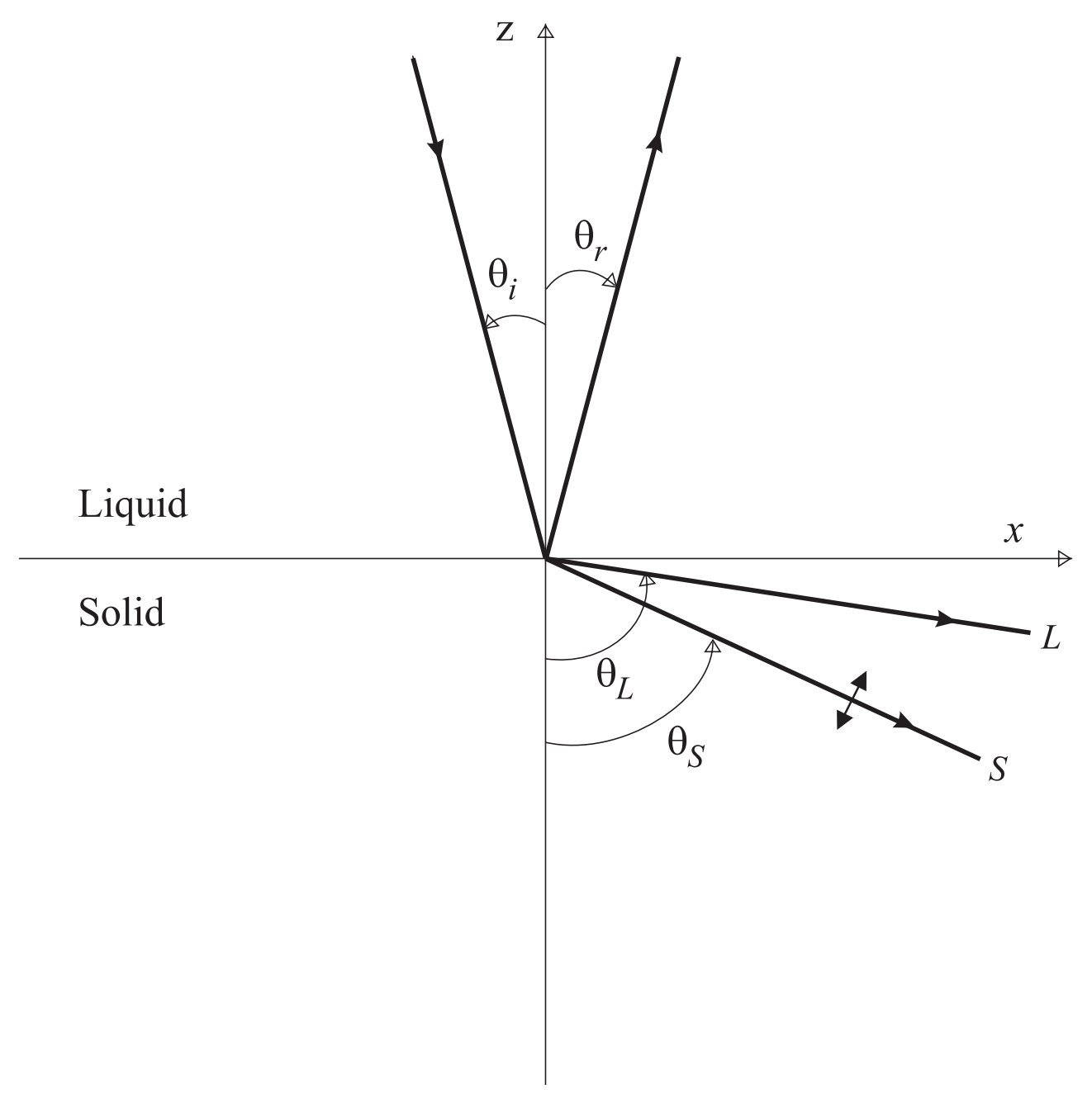

Figure 2.7: A liquid-solid interface with incident, reflected and refracted transmitted longitudinal (L) and shear (S) waves, after [11].

$$
\frac{\sin \theta_{i}}{V_{1}}=\frac{\sin \theta_{r}}{V_{1}}=\frac{\sin \theta_{L}}{V_{2 L}}=\frac{\sin \theta_{S}}{V_{2 S}}
$$

where $\theta_{S}$ is the angle of refraction for the shear wave. Critical angles exist when the angles of refraction $\theta_{L}$ and $\theta_{S}$ equal $90^{\circ}$ i.e. for both the longitudinal and shear waves. At and beyond these critical angles no energy is transmitted into the solid, instead the energy propagates as an interface 
wave. An angle of refraction equal to $90^{\circ}$ results in $\sin \theta=1$, from which the critical angle is calculated. The pressure reflection coefficient has an angular dependence, defined as [11]

$$
R_{p}=\frac{Z_{L} \cos ^{2} 2 \theta_{S}+Z_{S} \sin ^{2} 2 \theta_{S}-Z_{1}}{Z_{L} \cos ^{2} 2 \theta_{S}+Z_{S} \sin ^{2} 2 \theta_{S}+Z_{1}}
$$

as will the pressure transmission coefficients for the longitudinal and shear waves, defined as

$$
T_{L}=\frac{\rho_{1}}{\rho_{2}} \frac{2 Z_{L} \cos ^{2} 2 \theta_{S}}{Z_{L} \cos ^{2} 2 \theta_{S}+Z_{S} \sin ^{2} 2 \theta_{S}+Z_{1}}
$$

and

$$
T_{S}=\frac{\rho_{1}}{\rho_{2}} \frac{2 Z_{S} \sin ^{2} 2 \theta_{S}}{Z_{L} \cos ^{2} 2 \theta_{S}+Z_{S} \sin ^{2} 2 \theta_{S}+Z_{1}}
$$

respectively, where

$$
\begin{aligned}
Z_{1} & =\frac{\rho_{1} V_{1}}{\cos \theta_{i}} \\
Z_{L} & =\frac{\rho_{2} V_{L}}{\cos \theta_{L}} \\
Z_{S} & =\frac{\rho_{2} V_{S}}{\cos \theta_{S}}
\end{aligned}
$$

are the acoustic impedances for the incident, refracted longitudinal and refracted shear waves respectively. The form of these equations is similar to those for normal incidence except they are complicated by the need for angular dependence and mode conversion. For simplicity an effective impedance is defined [11]

$$
Z_{e f f} \equiv Z_{L} \cos ^{2} 2 \theta_{S}+Z_{S} \sin ^{2} 2 \theta_{S}
$$

from which the reflection coefficient (2.14) can be simplified to 


$$
R=\frac{Z_{\text {eff }}-Z_{1}}{Z_{\text {eff }}+Z_{2}}
$$

which has the same form as (2.8). Water and aluminium provide a good example of a liquid and solid with different impedances, presented in Table 2.1. Additional data are included for the critical angles for the longitudinal $\theta_{L}$, shear (SV) $\theta_{S}$ and Rayleigh $\theta_{R}$ waves for the water aluminium interface.

\begin{tabular}{ccccccccc}
\hline Material & $\rho$ & \multicolumn{3}{c}{ Velocity $\left[\mathrm{ms}^{-1}\right]$} & \multicolumn{3}{c}{ Critical angle $\left.{ }^{\circ}\right]$} & $\mathrm{Z}$ \\
\cline { 3 - 6 } & {$\left[\mathrm{kgm}^{-3}\right]$} & $V_{L}$ & $V_{S}$ & $V_{R}$ & $\theta_{L}$ & $\theta_{S}$ & $\theta_{R}$ & [MRayl] \\
\hline $\mathrm{H}_{2} \mathrm{O}$ & 1000 & 1480 & - & - & - & - & & 1.5 \\
$\mathrm{Al}$ & 3000 & 6300 & 3100 & 2894 & 13.6 & 28.5 & 30.8 & 18.9 \\
\hline
\end{tabular}

Table 2.1: Properties of Water and Aluminium with critical angles for the boundary, after [11].

The angular dependence of the reflected energy is often measured using a goniometer and can provide a means to determine the velocities of acoustic waves in a sample. The theoretical result of such a measurement for a water-aluminium interface is displayed in Fig. 2.8(a). For angles less than the longitudinal critical angle $\theta_{L}=13.6^{\circ}, 70 \%$ of energy is reflected, between $20 \%$ and $30 \%$ is transmitted as a refracted longitudinal wave and less than $10 \%$ is transmitted as a refracted shear wave. At the longitudinal critical angle the energy associated with the transmitted longitudinal wave goes to 0 and remains at 0 for higher angles, hence no more energy is transmitted as a longitudinal wave. The energy associated with the transmitted shear wave also goes to 0 and the reflected energy increases to $100 \%$, however these last two phenomena occur only at this precise angle. For angles greater than $\theta_{L}$ and less than $\theta_{S}$ the energy associated with the shear wave rapidly increases to between $40 \%$ and $60 \%$ of the total energy resulting in the generation of a large amplitude shear wave. As a result the reflected 
energy decreases. At the shear critical angle the transmitted energy again goes to 0 and all the energy is reflected. For angles greater than the shear critical angle all of the energy is reflected with $|R|=1$, seen in Fig. 2.8(b).

This total reflection is counterintuitive because a Rayleigh wave is generated at the Rayleigh critical angle (discussed above) and energy is required to generate this Rayleigh wave hence some incident energy must be 'transmitted' and not reflected. To explain why total reflection is true a non-specular reflection must be introduced. As explained above specular reflections follow the rules of geometric ray tracing. A non-specular reflection is where the reflected ray is displaced laterally [11], seen in Fig. 2.9.

For critical angle Rayleigh wave generation there is partial specular reflection with the remainder of energy generating the leaky Rayleigh wave. For an ideal, non-attenuating solid all of the energy used to generate the leaky Rayleigh wave will eventually be leaked as a non-specular reflection, as the leaky Rayleigh wave propagates along the surface. This confirms the total reflection $|R|=1$. However, no solid is ideal and attenuation of the Rayleigh wave in the solid will occur resulting in less than total reflection. This reduction in reflected energy is observed experimentally as the dashed line in the inset of Fig. 2.8(b), centred at the Rayleigh angle $\theta_{R}$. This is called the Rayleigh dip [11]. For a solid with small but critical attenuation, the Rayleigh dip is deep and narrow, and for high attenuation the Rayleigh dip is shallow and broad.

The phase of the reflection coefficient is close to $0^{\circ}$ for angles below and including the shear critical angle $\theta_{S}$. Between $\theta_{S}$ and $\theta_{R}$ the phase rapidly rises to $180^{\circ}$, in phase with the incident wave. This rapid change is again observed for angles above $\theta_{R}$, where the phase flips to $-180^{\circ}$. This is observed experimentally with the specular reflection having a phase of $0^{\circ}$ and the non-specular reflection having a phase of $180^{\circ}$ (the same as the 
incident wave). This phase mismatch results in a phenomenon called the Schoch displacement. For a finite width incident beam at the Rayleigh critical angle, the specularly reflected beam will have the same beam width. On the surface the incident beam will cover a finite region over which a Rayleigh wave will be generated but no energy will be leaked. Past this region energy will start leaking with a phase $180^{\circ}$ relative to the specularly reflected beam. Over the small region where the specular and nonspecular reflected beams meet the two out of phase beams will destructively interfere resulting in the null region [11, 14] seen in Fig. 2.9. As a result of this null region the two reflected beams will become easy to distinguish, the specular reflection with its finite beam width and the nonspecular reflection with its wider beam width and decaying amplitude. The cause of the Schoch displacement was verified experimentally [22] by placing a blocker on the surface of the solid in the null region to block the specular beam. Only the Rayleigh wave travelled 'under' the block and the only energy present on the other side of the block was due to the leaky Rayleigh wave. 


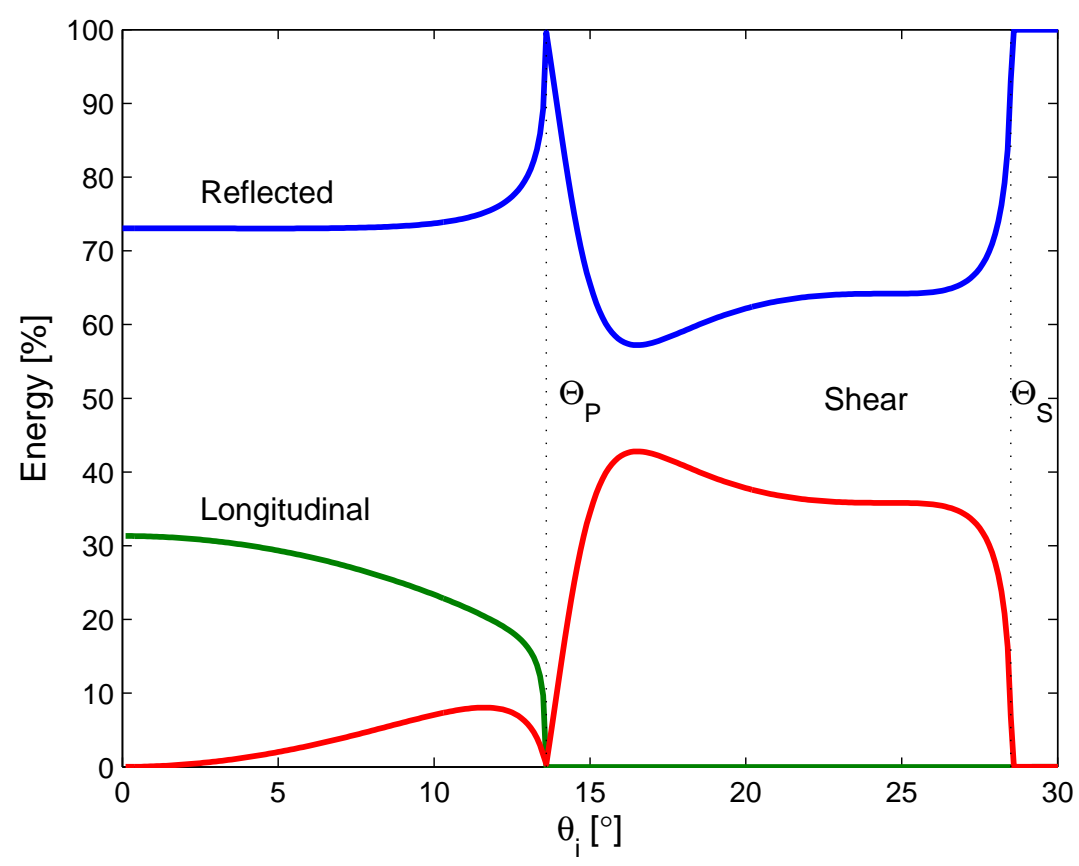

(a) Angular Dependence of the Reflected and Transmitted Energy at a Water-Aluminium Interface.

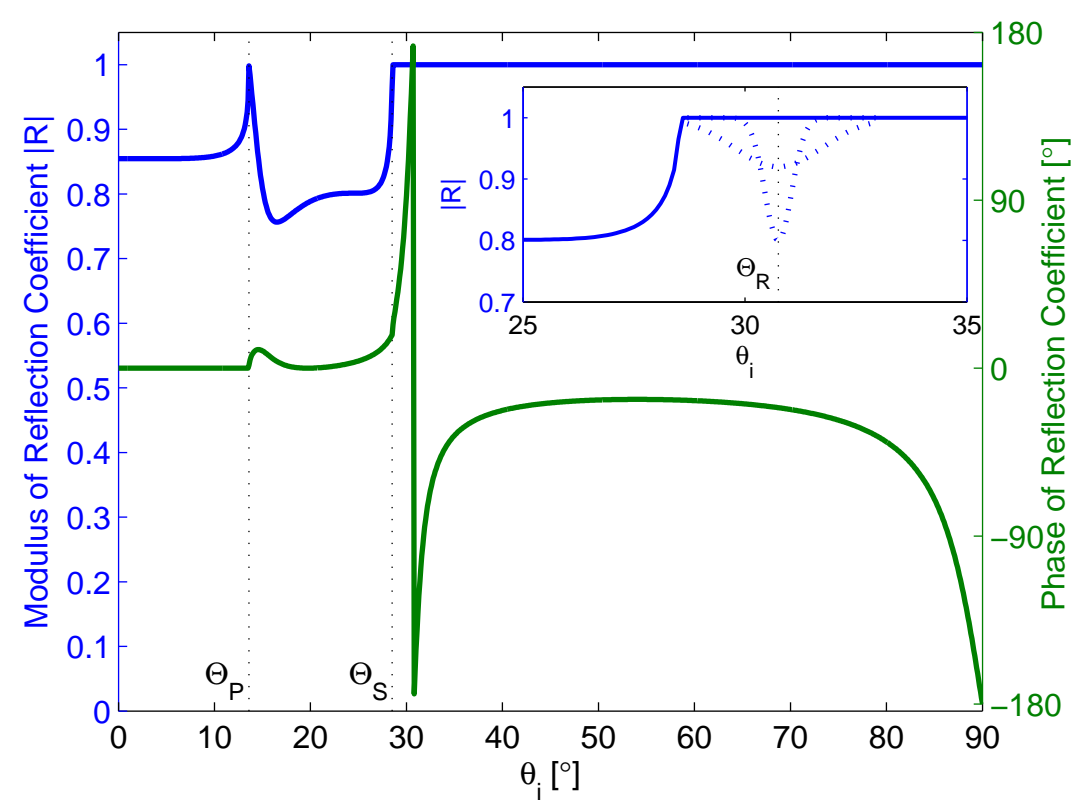

(b) Angular Dependence of the Reflection Coefficient for a WaterAluminium Interface.

Figure 2.8: Angular variation of the reflection coefficient of aluminium immersed in water. 


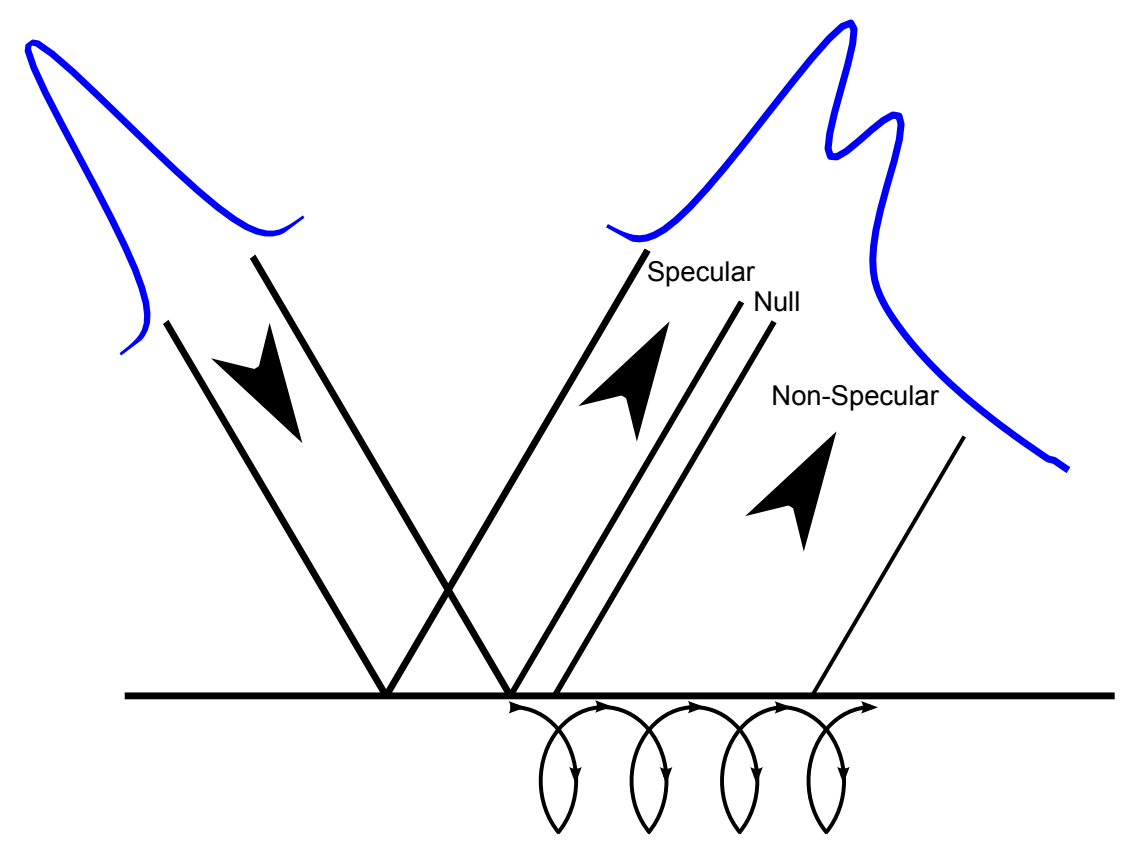

Figure 2.9: Specular reflection interfering with non-specular reflection from leaky Rayleigh wave resulting in the Schoch displacement phenomenon. 


\subsection{Attenuation}

As acoustic waves propagate through media they are attenuated with the causes attributed to the two separate processes of absorption and scattering.

\subsubsection{Absorption}

Absorption is the transfer of acoustic energy into random thermal energy. This loss of energy is caused by three processes [11, 13]; viscosity, thermal conductivity and internal molecular energy transfers. Viscous losses occur when adjacent parts of the medium move relative to each other i.e. from shear deformation. Thermal conduction losses occur when energy transfers from compression regions at high temperature to rarefaction regions of low temperature. Internal molecular energy transfers involve the kinetic energy of the particles converting to structural rearrangements and rotational/vibrational motion of polyatomic molecules. The absorption coefficient for a medium is defined $[11,13]$

$$
\alpha_{a b s r}=\frac{\omega^{2}}{2 \rho v^{3}}\left(\frac{4}{3} \eta_{\text {shear }}+\eta_{\text {bulk }}+\frac{(\gamma-1) \kappa}{C_{p}}\right)
$$

where $\omega$ is the angular frequency, $\rho$ is the density, $v$ is the acoustic velocity, $\eta$ is the viscosity, $\gamma$ is the specific heat ratio, $\kappa$ is the thermal conductivity and $C_{p}$ is the specific heat. It is clear that absorption is sensitive to and will rise rapidly with frequency because of the $\omega^{2}$. Absorption is observed as a reduction in amplitude $A$ of a signal as it propagates a distance $z$ through a medium

$$
A=A_{0} \exp (-\alpha z)
$$

where $A_{0}$ is the initial amplitude and $\alpha$ is the attenuation coefficient. The frequency dependent absorption for water at $20^{\circ} \mathrm{C}$ is seen in Fig. 2.10. Absorption will have a significant effect when immersion transducers are 


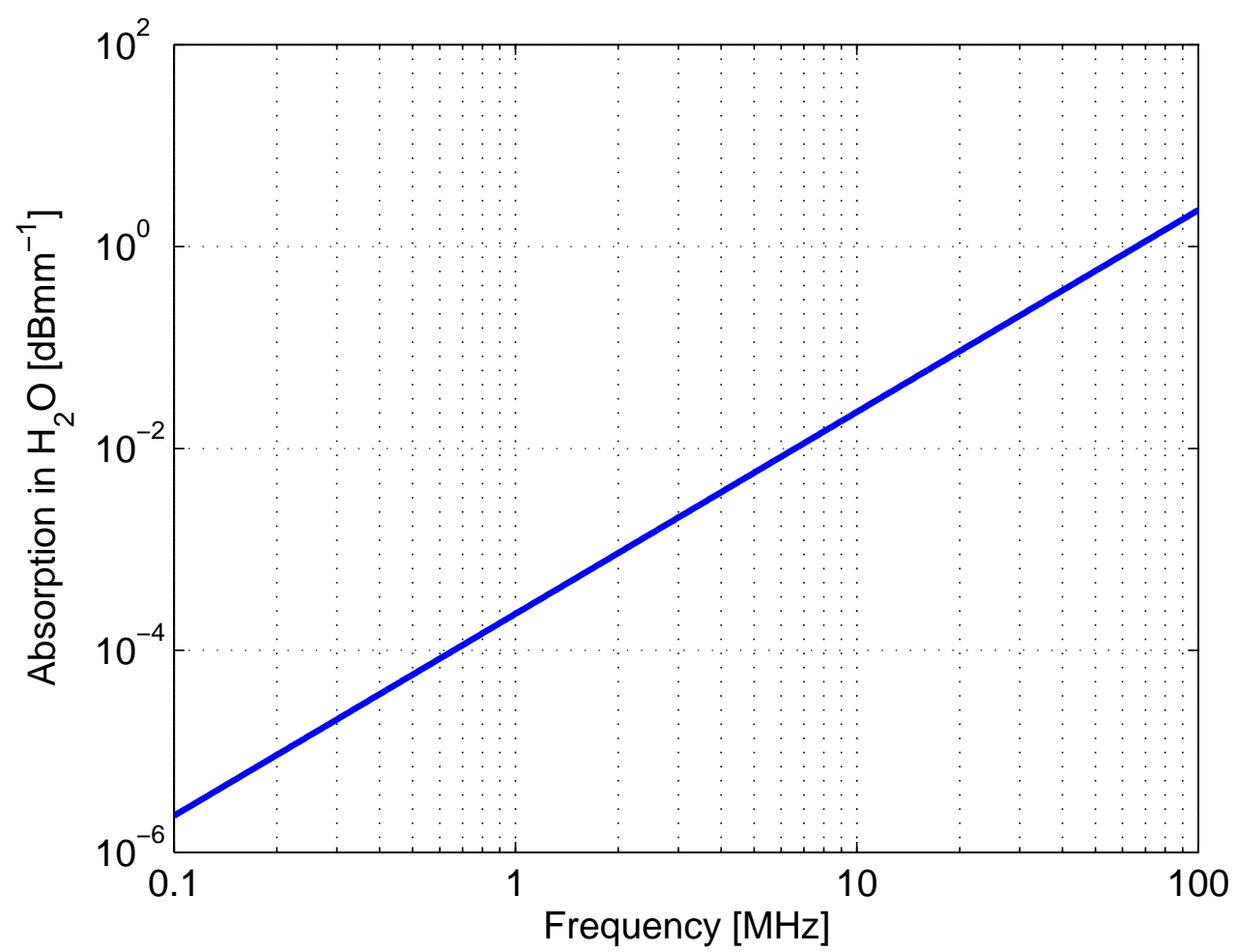

Figure 2.10: Absorption in water for the $\mathrm{MHz}$ range.

used as their focal lengths are typically $20 \mathrm{~mm}[23,24]$. At $10 \mathrm{MHz}$ for a focal length of $20 \mathrm{~mm}$ the absorption for a pulse-echo measurement is $0.9487 \mathrm{~dB}$, where as at $50 \mathrm{MHz}$ the absorption is $22.6204 \mathrm{~dB}$, a drastic decrease in signal amplitude.

\subsubsection{Scattering}

As with EM waves acoustic waves have their energy partially redirected when they interact with an object of different impedance. Scattering is an extension of reflection theory and is dependent on the size, shape and orientation of the scatterer and the wavelength $\lambda$. By comparing the $k=$ $\frac{2 \pi}{\lambda}$ and scatterer size $a$, acoustic scattering can be classified into one of three 
categories: scatterers much larger than a wavelength, much less than a wavelength and comparable to the wavelength. The product $k a$ is often referred to when describing scattering phenomena [11, 15, 16]. For this explanation it is assumed that the scatterer size $a$ is kept constant while the frequency/wavelength/wavenumber is varied. Scattering differs slightly between cylinders and spheres [11] however the fundamental ideas are consistent.

\section{Specular Scattering}

When the wavelength is much smaller than the scatter $(k a \gg 1)$ the scattering is specular. Due to the length scales concerned the scatterer can be approximated as a flat surface so that ray theory can be applied.

\section{Diffusive Scattering}

Diffusive scattering is the other extreme, where the wavelength is much larger than the scatterer $(k a \ll 1)$. The acoustic energy will scatter over a wide range of angles and very little energy will be reflected back to the source. This is often called Rayleigh scattering (after Lord Rayleigh's paper on why the sky is blue [25]) and is found to depend on frequency to the fourth power.

\section{Diffractive Scattering}

Diffractive scattering describes the phenomena associated with a scatterer of approximately the same size as the wavelength $(k a \sim 1)$. In this region the scattering intensity is found to vary significantly with small changes in $k$ [11]. Polar plots in Fig. 2.11 display the drastic change in the directional dependence of the scattered energy, here the incident wave is from the right. 

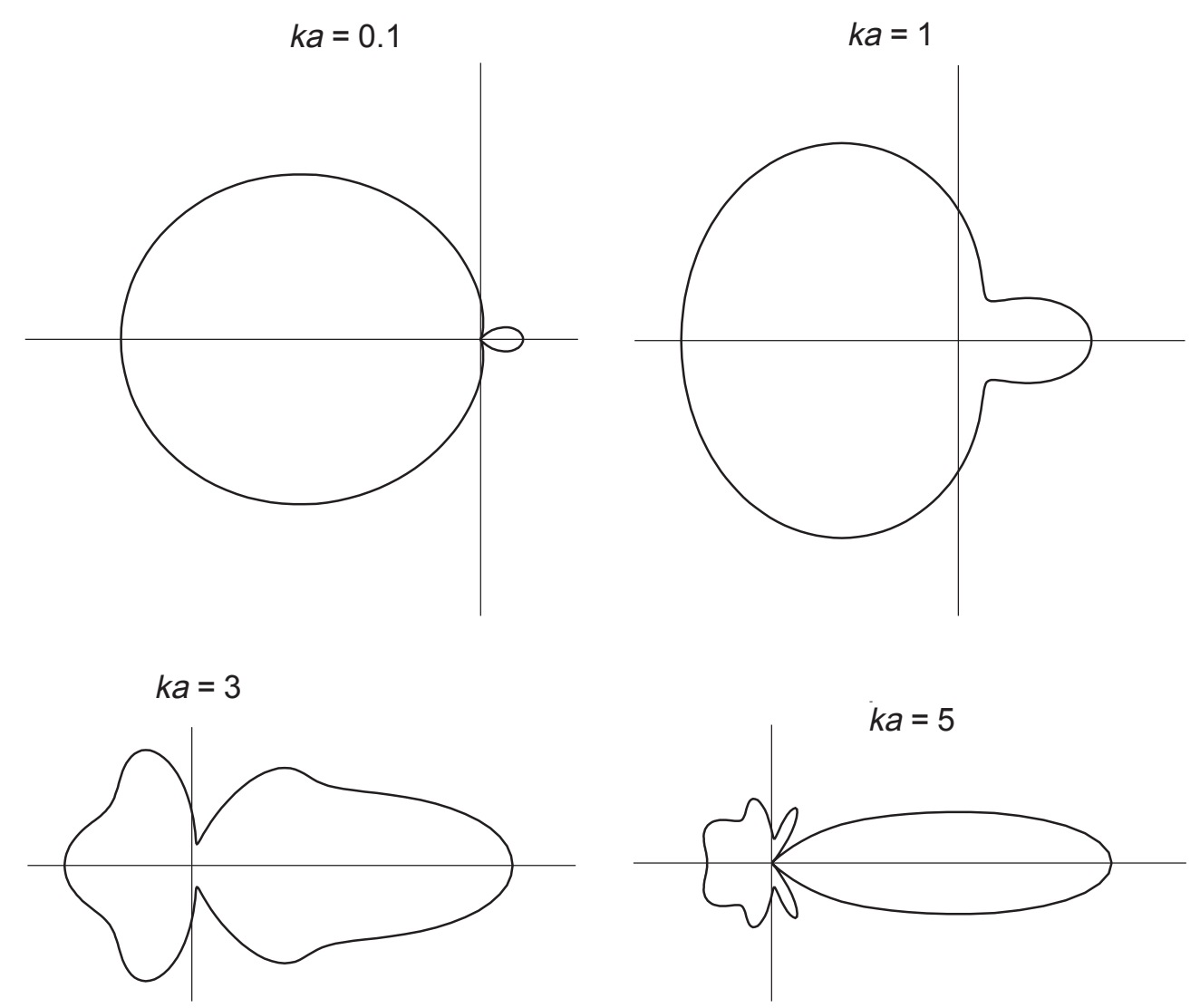

Figure 2.11: Scattered energy for a rigid sphere diffractive scattering, after [11]. The incident wave is from the right. 


\section{Chapter 3}

\section{Experimental Setup}

This chapter details the high frequency ultrasonic equipment, techniques and electronics employed to perform the experiments associated with this work. Section 3.1 details the construction, properties and frequency response of the ultrasonic immersion transducers used. Section 3.2 describes the Pulse-Echo and Pitch-Catch methods of ultrasonic inspection. A brief functional description of the high frequency electronics required to operate the transducers is presented in Section 3.3.

\subsection{Transducers}

Transducers are devices that convert energy from one form to another with ultrasonic transducers converting between electrical and mechanical. These transducers take advantage of the piezoelectric effect where a crystal will deform in response to an applied potential, acting as a transmitter. The use of an alternating potential will cause the crystal to deform at the same frequency, useful for generating acoustic waves at a desired frequency. The opposite is also true, a mechanically deformed crystal will produce a corresponding potential allowing a transducer to be used as a receiver. All transducers are designed to have a maximum response in a certain frequency range and are assigned a centre frequency and band- 
width.

\subsubsection{Construction of Piezoelectric Transducers}

The typical construction of an ultrasonic contact transducer is presented in Fig. 3.1(a). The housing is often metallic for strength and wear resistance. The three main parts of a transducer are the active element, backing material and wear plate.

\section{Active Element}

Materials commonly used as active elements are polarized ceramics of the lead zirconate family $[11,1]$ as they can be cut along different principle axes to produce both longitudinal and shear waves. Other materials include piezo-polymers and-ceramics or composites that can provide desired properties for specific applications. Electrodes are placed on the top and bottom sides of the active element to provide the potential difference, hence the active element has similar properties to a capactior.

\section{Backing}

The backing of a transducer is a with significant acoustic attenuation used to dampen the wave propagating backward into the transducer body. A heavily damped transducer has a backing that is impedance matched to the active element and thus produces low energy pulses. These pulses are short resulting in improved axial resolution and a large bandwidth. The opposite is true for transducers with low damping, as the acoustic impedance mismatch is greater more energy is reflected from the backing resulting in a higher energy pulse. The pulse length is longer which decreases resoution and the bandwidth narrows. 


\section{Wear Plate}

The wear plate on the transducer is incorporated in order to protect the active element from scratching and serve as an impedance matching layer. In contact transducers the wear plate is one quarter of a wavelength $(\lambda / 4)$ thick, designed for constructive interference of reverberating waves in the wear plate, resulting in a larger signal. Immersion transducers often use a much longer wear plate made of quartz called a delay line. The delay line allows the use of long pulses and enables the active element to 'ring down' before an echo returns. One advantage of a delay line in an immersion setup is the acoustic matching improvement between water and active element, resulting in improved energy transfer. Delay lines often have a lens cut or ground into their end face which causes the ultrasound to refract at the water boundary and focus the beam, increasing the intensity, seen in Fig. 3.1(b). Water provides a uniform coupling between the transducer and the sample, enabling samples with complex shapes to be investigated.

\section{Beam Properties}

The $-6 \mathrm{~dB}$ beam diameter [1] of a focussed immersion transducer is defined as

$$
B D(-6 d B)=1.02 \frac{F c}{f D}
$$

where $F$ is the focal length, $c$ is the velocity in the immersion liquid (water), $f$ is the frequency and $D$ is the diameter of the active element, seen in Fig. 3.1(b). The lateral resolution of a transducer is defined as the $6 \mathrm{~dB}$ beam diameter at the focal point [1]. For two distinct reflectors to be resolved laterally the distance between the two must be greater than the lateral resolution. This ensures the reflectors are never in the beam at the same instance and prevents mixing of signals, hence resolving the two reflectors independently. For two distinct reflectors positioned along the 
beam axis to be fully resolved the reflected acoustic pulses must not overlap. The smallest separation of two such reflectors is half the spatial pulse length (SPL), the distance in space that a pulse covers. The corresponding axial resolution is

$$
R=\frac{c N}{2 f}
$$

where $N$ is the number of cycles in the pulse.

\subsubsection{Transducer Properties}

Five Olympus Panametrics immersion transducers of three different centre frequencies were used in this work. One pair had nominal centre frequencies of $50 \mathrm{MHz}$ while another pair had centre frequencies of $20 \mathrm{MHz}$ and a single transducer was centered at $10 \mathrm{MHz}$. Specification sheets were supplied with the devices [23, 24, 26] from which the lateral and axial resolutions of the three transducer models were calculated, seen in Fig. 3.2.

The spec sheets also presented the results of tests that followed the standard method of the American Society for Testing and Materials document E 1065 "Standard Guide for Evaluating Characteristics of Ultrasonic Search Units" [27]. The technique used to characterise each transducer was the 'Shock' technique [28]. A pulser unit generated a pulse of very short duration, quick rise time $(<2 \mathrm{~ns})$ and large amplitude $(-180 \mathrm{~V})$. This pulse provided a broad spectrum of energies and caused the active element to deform and resonate, or 'ring down', at its resonant frequency, which generated an acoustic pulse. The shape of acoustic pulse was the convolution of the voltage spike shape with the impulse response of the transducer. The transducer was immersed in water and a steel ball placed inside the focal region that acted as an equal phase reflector. The acoustic pulse was reflected back to the transducer and deformed the active element which generated a time varying voltage. This voltage was the 


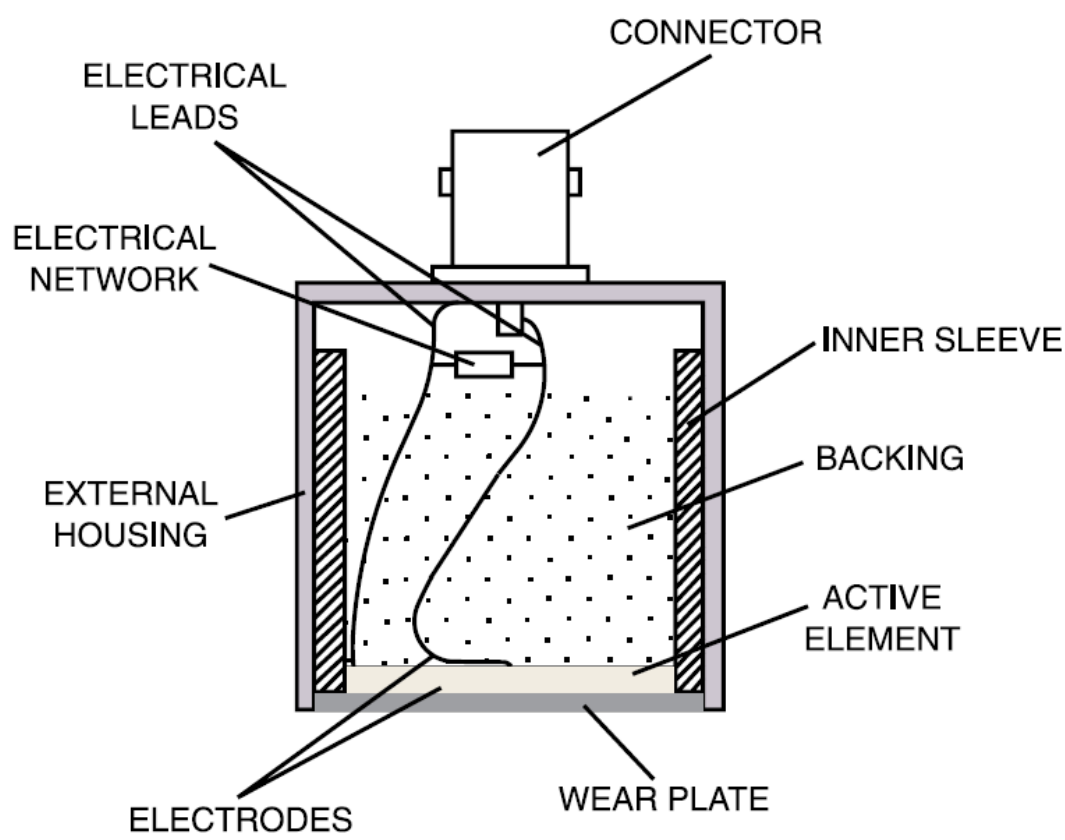

(a) Cross-section of the construction of ultrasonic contact transducer, after [1].

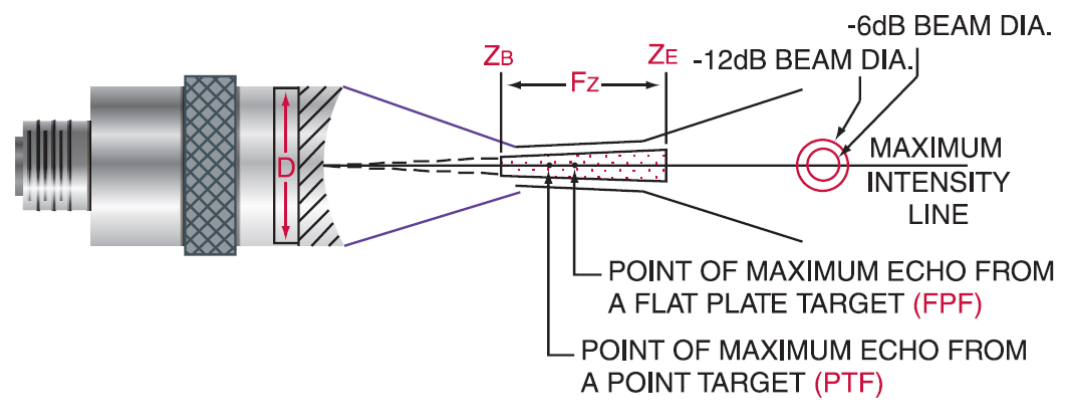

$$
\begin{array}{ll}
Z_{B}= & \text { Beginning of the Focal Zone } \\
F_{Z}= & \text { Focal Zone } \\
Z_{E}= & \text { End of the Focal Zone } \\
D & =\text { Element Diameter }
\end{array}
$$

(b) The -6dB beam diameter, focal length and corresponding focal zone of a focussed immersion transducer, after [1].

Figure 3.1: Construction and beam properties of an ultrasonic transducer. 


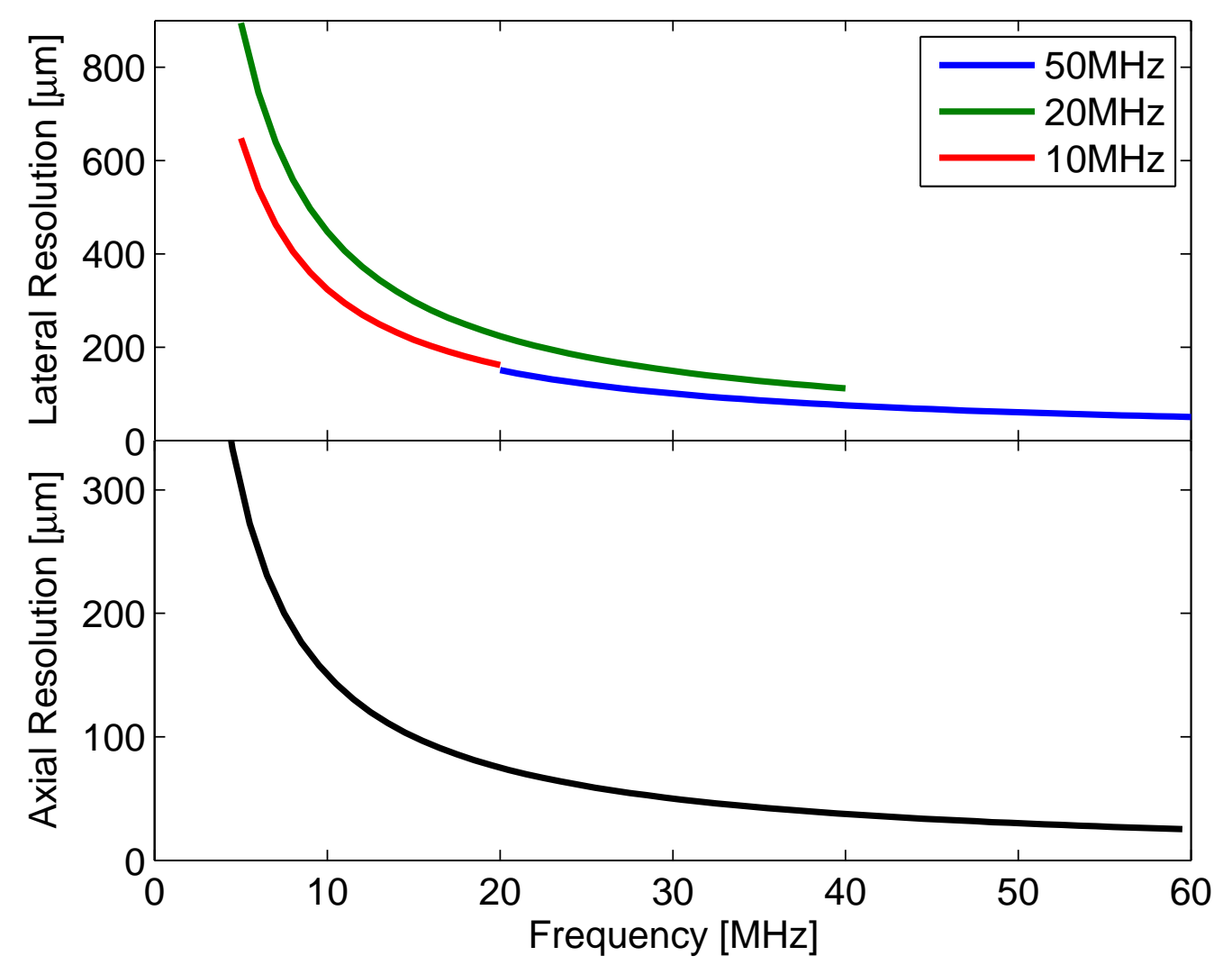

Figure 3.2: The lateral and axial resolution of the three transducers [1]. The axial resolution depends only on the frequency of the wave hence is independent of the individual transducer.

convolution of the reflected acoustic pulse with the impulse response of the transducer and was recorded on an oscilloscope, seen in Fig. 3.3(a). This recording was Fast Fourier Transformed (FFT) from which the frequency response of the transducer was acquired, seen in Fig. 3.3(b). From these frequency responses, the centre frequency and $-6 \mathrm{~dB}$ bandwidth of the transducers were determined. These values are tabulated along with other properties of the transducer in Table 3.1.

The shock technique provided an insight into the impulse response of the 


\begin{tabular}{ccccccc}
\hline $\begin{array}{c}\text { Model } \\
\text { Number }\end{array}$ & $\begin{array}{c}\text { Nominal } \\
\text { Freq. }\end{array}$ & $\begin{array}{c}\text { Centre } \\
\text { Freq. }\end{array}$ & BW & $\begin{array}{c}\text { Element } \\
\text { Diameter }\end{array}$ & $\begin{array}{c}\text { Delay } \\
\text { Line }\end{array}$ & $\begin{array}{c}\text { Focal } \\
\text { Length }\end{array}$ \\
\hline VHz & $\mathrm{MHz}$ & $\mathrm{MHz}$ & $\mathrm{mm}$ & $\mu \mathrm{s}$ & $\mathrm{mm}$ \\
\hline V390 & 50 & 45 & $27-61$ & 6.35 & 19.7 & 12.7 \\
V327 & 20 & 29 & $18-40$ & 6.35 & 2.2 & 17.8 \\
& 10 & 9.5 & $6-13$ & 9.525 & 0 & 20 \\
\hline
\end{tabular}

Table 3.1: Properties of Transducers, after [23, 24, 26].

transducer, in that the active element would oscillate and ring down close to the natural frequency. However this pulse echo measurement was a representation of the manufacturer's system, rather than the transducer's response in a typical Pulse-Echo measurement. During the shock testing high frequency water absorption was significantly reduced as the steel ball was located $6 \mathrm{~mm}$ from the transducer for the $50 \mathrm{MHz}$ and $20 \mathrm{MHz}$ devices, corresponding to a half or third of their focal length's, respectively. Whereas a typical measurement would have a reflector at the focal spot where the wave is more uniform. The shock excitation provided a broad range of energies however the spectrum of this pulse was not provided. Control of a pulse's spectrum is desired to reduce water absorption, minimise scattering or change the resolution. This control can be achieved through the use of multiple cycle sinusoidal pulses centred at a chosen frequency, generated by a function generator. A pulse regularly used in this research was a 5 cycle sinusoid with an envelope matching the Hann window [29], seen in the inset of Fig. 3.4. To measure the frequency response of the transducers in a typical pulse-echo setup, a smooth flat glass reflector was placed at the focal spot, which would increase the attenuation as the water path was longer. The centre frequency of the 5 cycle Hann sinusoid was varied and the amplitude voltage of the echo signal recorded. The frequency response of the transducers to the sinusoidal excitation is presented in Fig. 3.4.

The broadband nature of the $50 \mathrm{MHz}$ device is highlighted in this mea- 
surement with the maximum response between $25 \mathrm{MHz}$ and $35 \mathrm{MHz}$, at the lower end of the quoted range, while the response fell off very sharply below $20 \mathrm{MHz}$. The $20 \mathrm{MHz}$ transducer suprisingly had a weaker response than the $50 \mathrm{MHz}$ in the range above $15 \mathrm{MHz}$. However the response of the $50 \mathrm{MHz}$ below $15 \mathrm{MHz}$ was not measured as the signal quality detereorated such that the signal was not an accurate representation of a sinusoid. Whereas the $20 \mathrm{MHz}$ retained signal quality at these lower frequencies. In contrast the $10 \mathrm{MHz}$ had a very sharp peak in its response and could be used down to $5 \mathrm{MHz}$ before the signal quality detereorated. These results guided the choice of transducer for a particular frequency range; for all measurements above $20 \mathrm{MHz}$ the $50 \mathrm{MHz}$ transducers would be employed while lower frequency measurements required a combination of the $10 \mathrm{MHz}$ and $20 \mathrm{MHz}$ transducers, dependent on the particular measurement. 


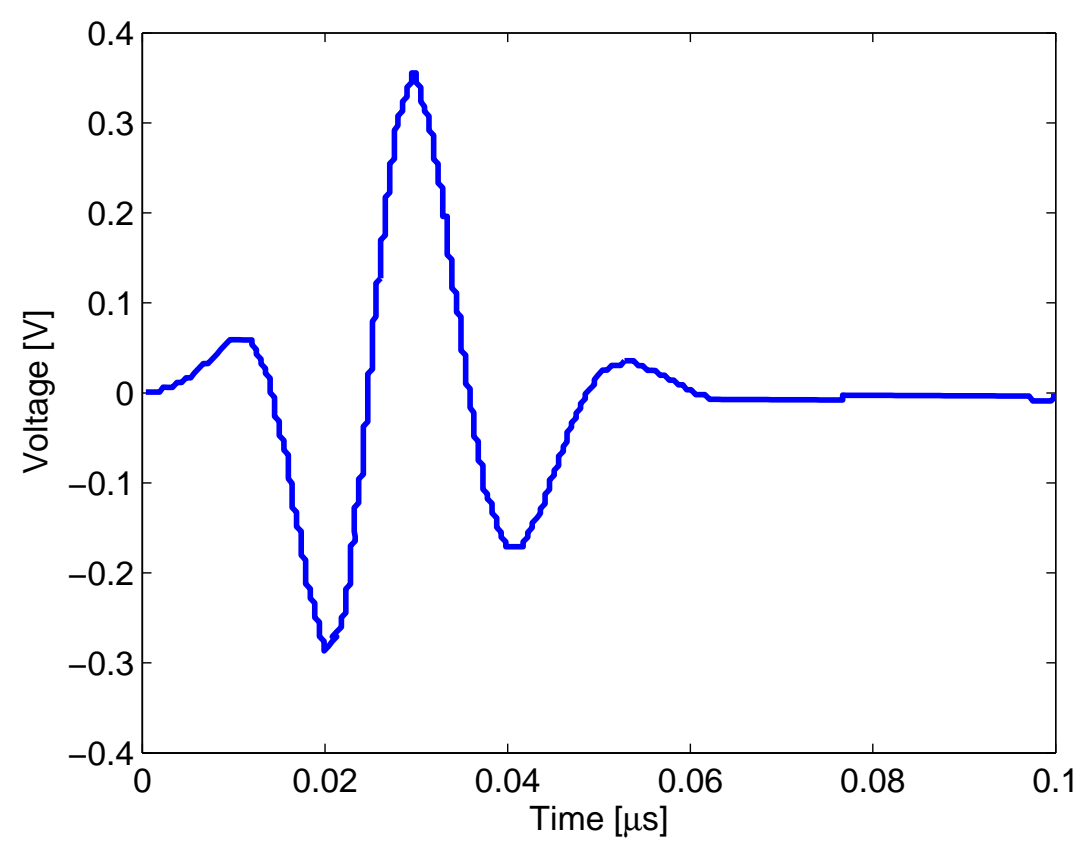

(a) Response of 50MHz transducer to ASTME1065 'Shock' technique - a voltage spike applied to the transducer aimed at a steel ball in water in a Pulse-Echo setup. Data from manufacturer's testing [23].

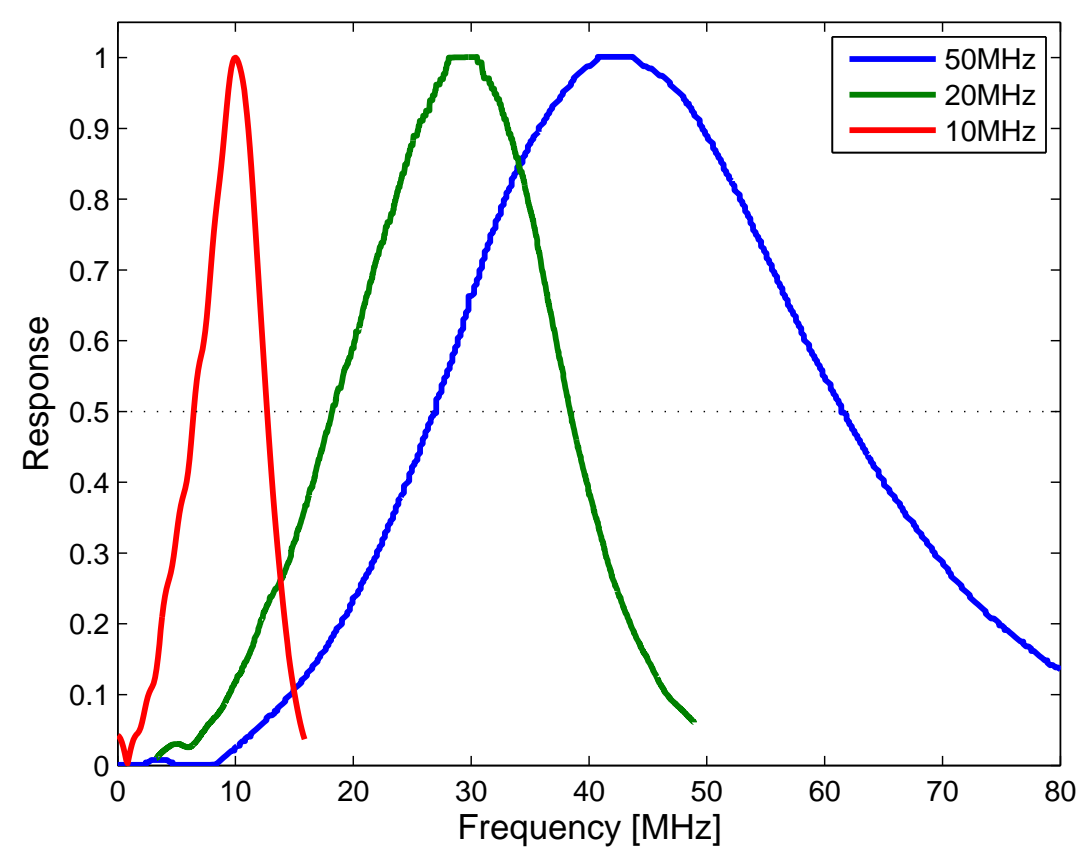

(b) Normalised frequency response of $50 \mathrm{MHz}, 20 \mathrm{MHz}$ and $10 \mathrm{MHz}$ transducers obtained via FFT of recorded waveform.

Figure 3.3: Results of shock testing of the three Olympus Panametrics transducers used in this research. 


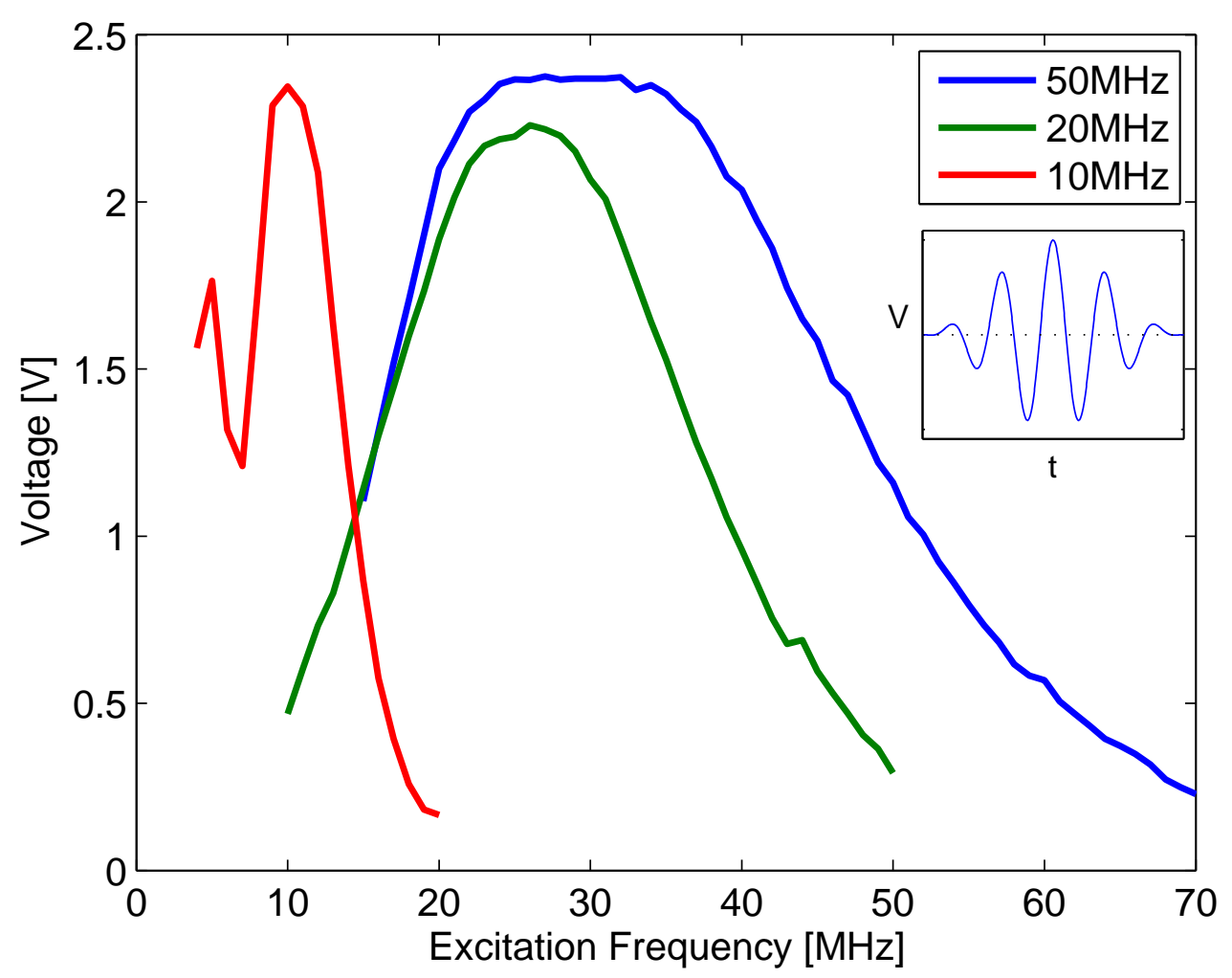

Figure 3.4: Measured response of transducers with flat glass reflector at focal length. The excitation pulse was a Hann windowed 5 cycle sinusoid (inset). The centre frequency was varied and the peak voltage recorded for each frequency. 


\section{Transducer Electrical Impedance}

The electrical impedance (magnitude and phase) of each transducer was measured using a Agilent 4291B impedance analyser with a bandwidth of $1 \mathrm{MHz}-1.8 \mathrm{GHz}$. The 50MHz transducers were purchased in 2006 and the impedance was measured at the time by other researchers [2]. The $20 \mathrm{MHz}$ transducers were purchased in mid 2011 and their impedance measured upon arrival. The two $50 \mathrm{MHz}$ devices displayed very similar characteristics, seen in Fig. 3.5, and have an impedance of $33 \Omega$ at $50 \mathrm{MHz}$. The small oscillatory nature of the plots between $25 \mathrm{MHz}$ and $75 \mathrm{MHz}$ is due to the element responding to reverberations in the delay line from earlier in the measurement. These reverberations highlight the broad range of frequencies over which the transducer is active. Near DC the magnitude is $50 \Omega$ and the phase $0^{\circ}$ due to a $50 \Omega$ resistor placed across the input. This resistor provides cable matching and results in a more resistive and less capacitive device that is easier to drive as less energy is stored in the electric field. Since the resistor changes the electrical network these plots show a distinct change from the $1 / f$ impedance plots typical of a capacitor.

The impedance of the $20 \mathrm{MHz}$ devices is presented in Fig. 3.6, where the DC value of $50 \Omega$ signifies the presence of a resistor network. With increasing frequency there is a reduction in magnitude down to $45 \Omega$ at $20 \mathrm{MHz}$. The delay line reverberations are present however the corresponding range of frequencies is shifted and reduced due to the limited bandwidth of the transducer $(18-39 \mathrm{MHz})$.

Finally the impedance of the $10 \mathrm{MHz}$ device was found to be very close to that of an ideal capacitor, seen by the $1 / f$ response at frequencies below the operating region of $\sim 10 \mathrm{MHz}$. This was due to the lack of a discrete resistor which in the $50 \mathrm{MHz}$ and $20 \mathrm{MHz}$ devices forced the near DC impedance to $50 \Omega$. In the operating region the impedance is $38 \Omega$. 


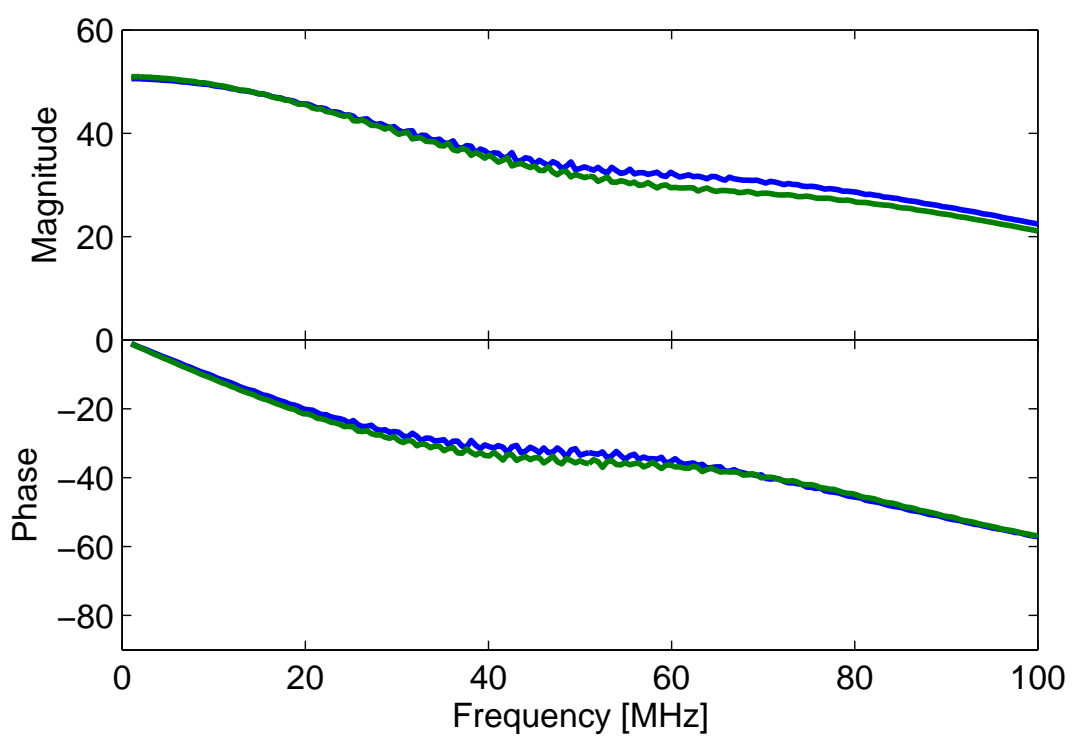

Figure 3.5: Magnitude and Phase [2] of Olympus Panametrics V390 $50 \mathrm{MHz}$ Immersion Transducer.

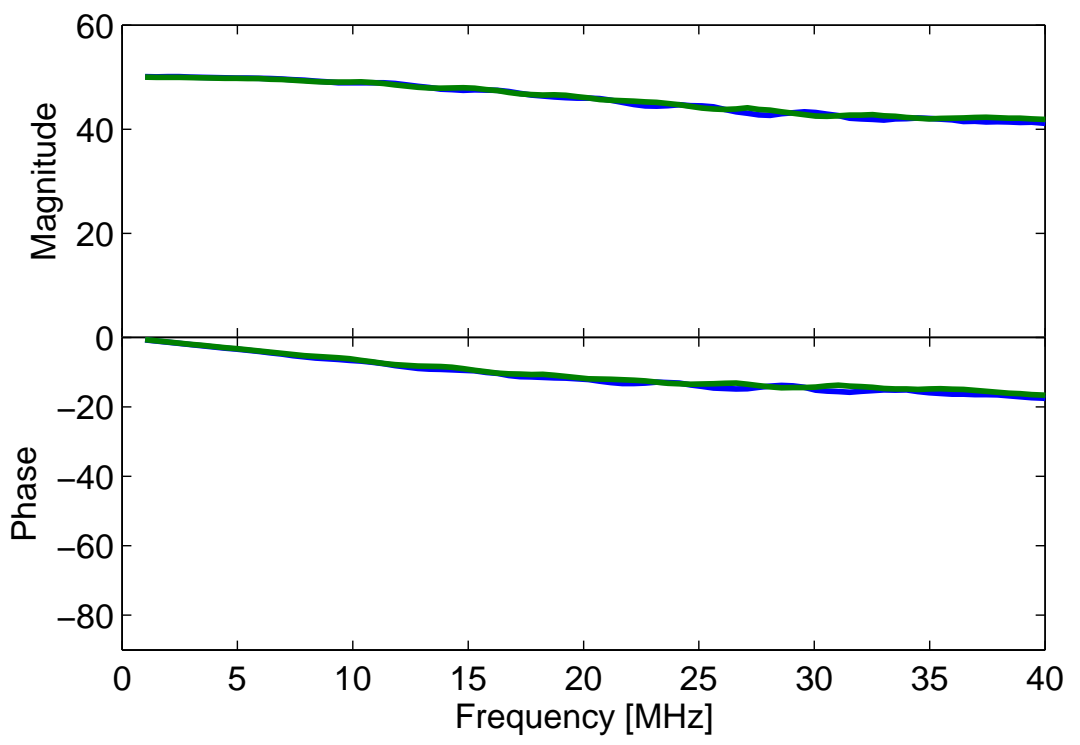

Figure 3.6: Magnitude and Phase of Olympus Panametrics V372 20MHz Immersion Transducer. 


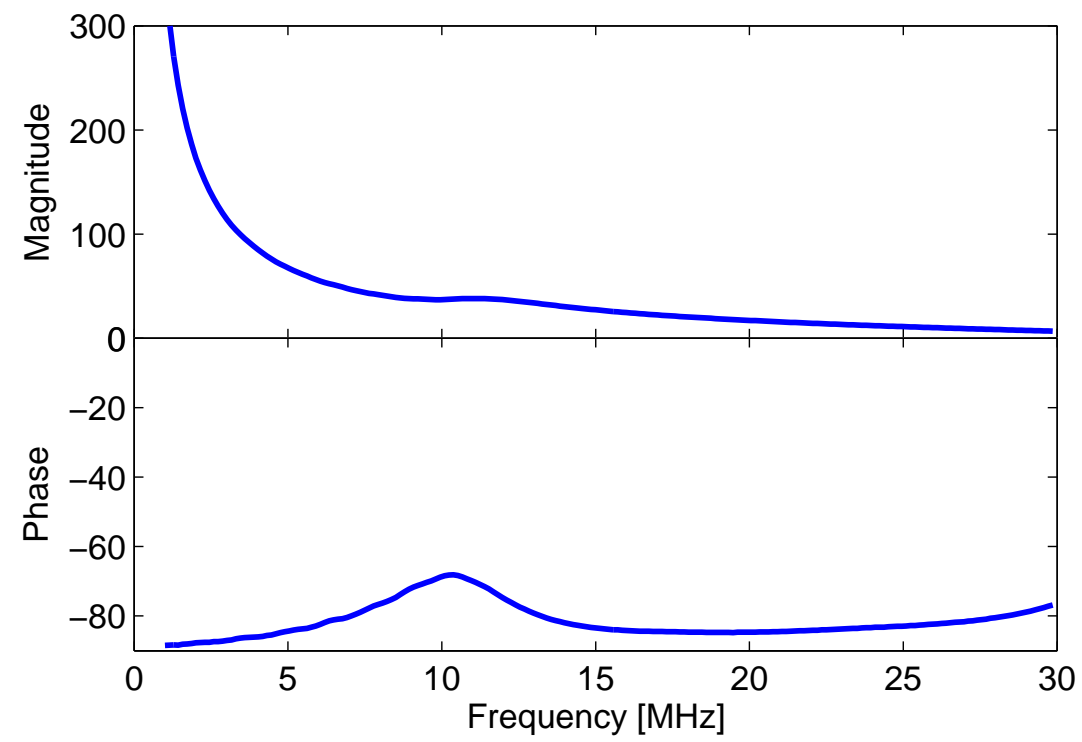

Figure 3.7: Magnitude and Phase of Olympus Panametrics V327 10MHz Immersion Transducer. 


\subsection{Ultrasonic Techniques}

All ultrasonic measurements fall into one of two categories, pulse-echo or pitch-catch. Both types of measurements can be employed independently or simultaneously to determine properties of a sample. This section details the techniques for the two measurement types and describes how sample information can be obtained from each technique.

\subsubsection{Pulse-Echo Measurements}

Pulse-echo measurements are the simplest ultrasonic measurements and require only one transducer. A simple pulse-echo measurement is described with reference to Fig. 3.8 which shows an immersion measurement. A pulse excites a transducer and an ultrasonic wave is emitted. This wave is reflected at regions of impedance mismatch and the reflections arrive back at the transducer. The first reflection to return to the transducer from the sample will be the front wall reflection $\left(t_{r 1}\right)$. Any reflections from inside a sample i.e. a crack or bubble, are potential intermediate reflections. Back wall reflections are those from the far side of a sample $\left(t_{r 2}\right)$. The time of flight (TOF) is the difference in time between the excitation pulse and the arrival of a reflected pulse. This can easily be measured on an oscilloscope. From TOF data distances or velocities can be calculated through $v=\frac{2 d}{t}$, taking into account the distance travelled is twice the thickness. To calculate a thickness the velocity in the sample must be known a priori, and vice versa. More complex methods exist [30] to measure a sample with unknown thickness and velocity. Such methods would be employed when a sample is flexible, fragile or thin, and the act of measuring the thickness with calipers may damage the sample. One of these methods will be covered in Section 3.2.3. 


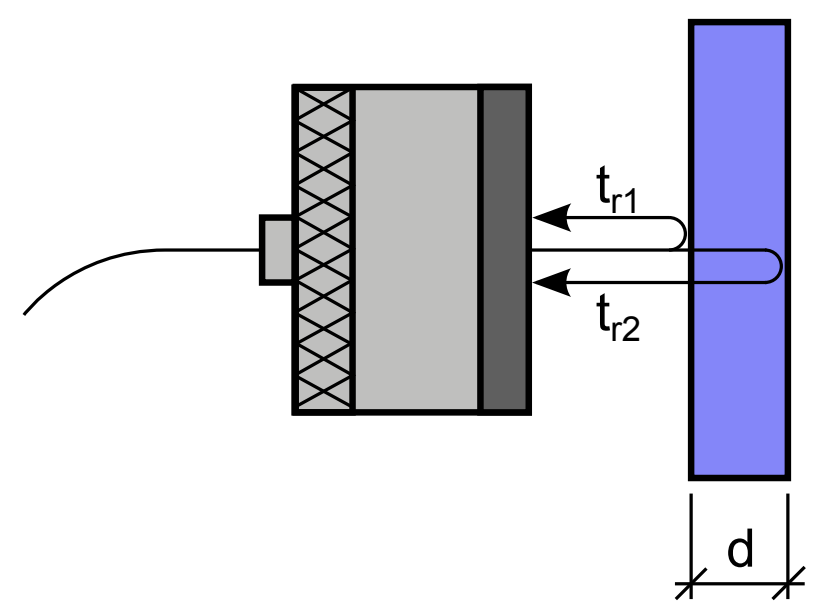

Figure 3.8: Ultrasonic pulse-echo immersion technique for determining a sample's thickness and/or velocity.

\section{Acoustic Impedance}

Pulse-echo measurements can be utilised to determine the unknown acoustic impedance of a sample immersed in water. Equation (2.8) can be rearranged to solve for the unkown impedance $Z_{2}$

$$
Z_{2}=\frac{Z_{1}\left(-R_{p}-1\right)}{R_{p}-1}
$$

where $Z_{1}$ is the known impedance of water. The reflection coefficient $R_{p}$ can be obtained experimentally using the following method. First the amplitude of the signal $t_{r 1}$ from Fig. 3.8 is recorded. The sample is then replaced by a reference sample with a smooth surface and known impedance/reflection coefficent i.e. glass [11]. The amplitude of the signal from the reference is recorded. The ratio of these amplitudes is multiplied by the reference reflection coefficient to obtain $R_{p}$. 


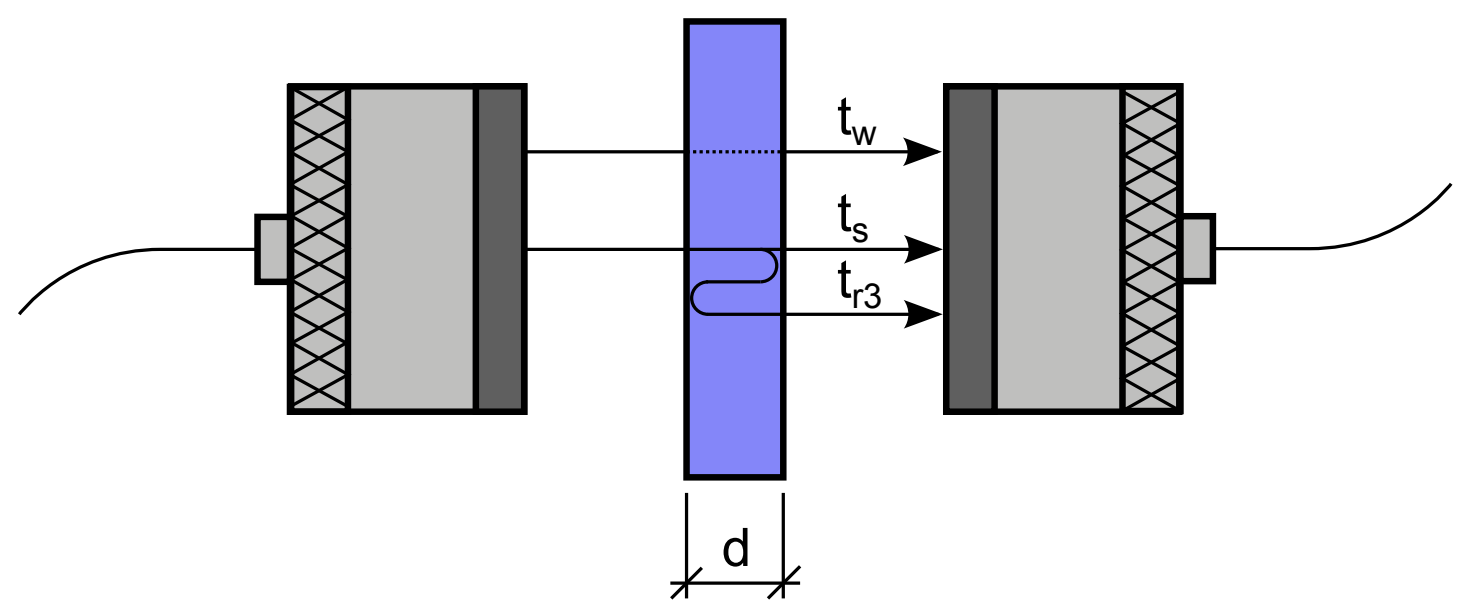

Figure 3.9: Ultrasonic pitch-catch immersion technique for determining a sample's thickness and/or velocity.

\subsubsection{Pitch-Catch Measurements}

Pitch-catch measurements require two transducers and are used to measure sample properties through which an ultrasonic pulse has passed, seen in Fig. 3.9. As with pulse-echo measurements, TOF data are measured with the sample present $\left(t_{s}\right)$ and removed $\left(t_{w}\right)$ i.e. the water path. Thickness or velocity values can then be calculated as before.

\section{Attenuation}

The attenuation coefficient $\alpha(f)$ of a material can be measured using a pitch-catch technique. A broadband pulse is transmitted through two identical samples that differ only in thickness (i.e. $d_{1} \& d_{2}$ ). The magnitude spectra for each recorded pulse $\left(A_{1}(f) \& A_{2}(f)\right)$ are calculated via the FFT. The attenuation coefficient over the range of frequencies present in the broadband pulse can be calculated as 


$$
\alpha(f)=\frac{-20 \log \left(\frac{A_{2}(f)}{A_{1}(f)}\right)}{d_{2}-d_{1}}
$$

\subsubsection{Simultaneous Pulse-Echo and Pitch-Catch Measure- ments}

Both pulse-echo and pitch-catch measurements require a prior knowledge of either the distance or velocity. Samples may be fragile, flexible or rough hence measuring their thickness with a pair of callipers may be difficult or damaging. Methods in the literature [30] exist to simultaneously determine distance and velocity for samples where neither velocity or thickness are known. These methods combine pitch-catch and pulse-echo data and require multiple reverberations or additional reflectors. One measurement technique used in this research involved measuring the reflection and transmission times from Fig. 3.10. The electrical connections were switched to record $t_{r 4}$. The unknown thickness and velocity are calculated

$$
\begin{aligned}
& d=\frac{v_{w}}{2}\left[\left(t_{w}-t_{r 1}\right)+\left(t_{w}-t_{r 4}\right)\right] \\
& v=v_{w} \frac{\left(t_{w}-t_{r 1}\right)+\left(t_{w}-t_{r 4}\right)}{\left(t_{s}-t_{r 1}\right)+\left(t_{s}-t_{r 4}\right)}
\end{aligned}
$$

where $v_{w}$ is the velocity of water. 


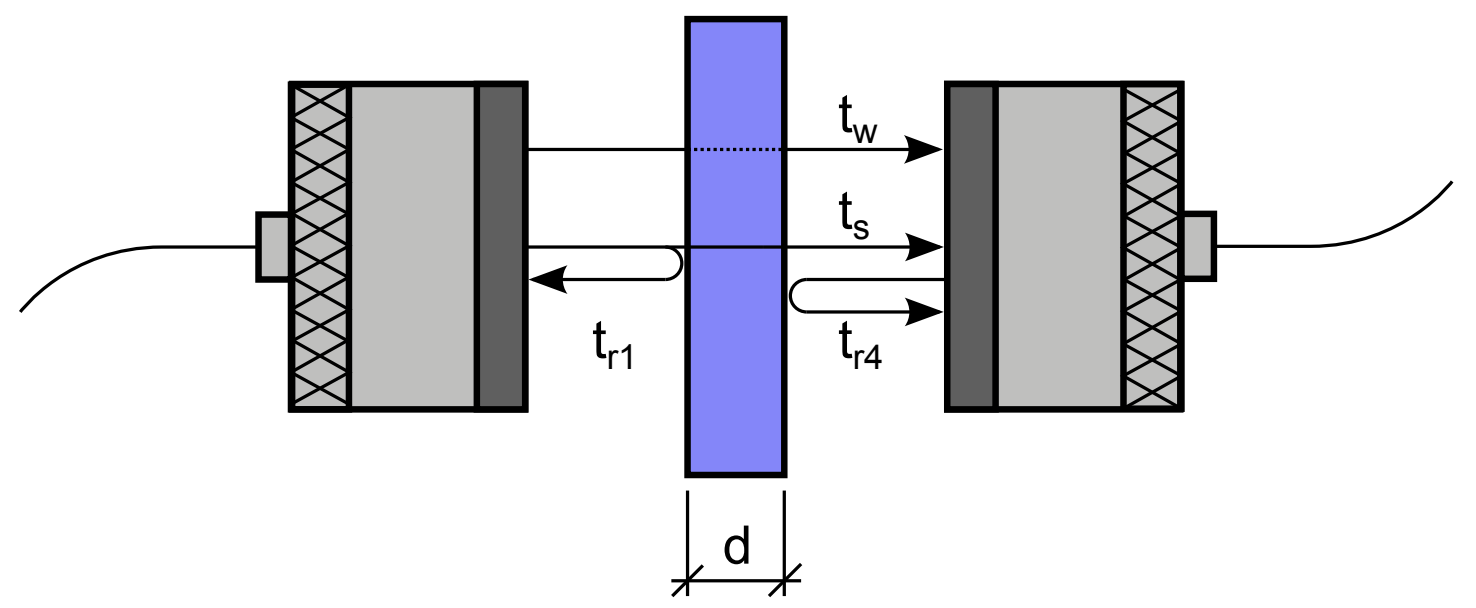

Figure 3.10: Simultaneous ultrasonic pitch-catch and pulse-echo immersion technique for determining a sample's thickness and velocity. 


\subsection{Electronics}

The electronics associated with the ultrasound system were configured to simultaneously operate in pulse-echo and pitch-catch modes between $10 \mathrm{MHz}$ and $100 \mathrm{MHz}$ allowing the three different types of immersion transducers to be swapped. The functionality is described below with reference to Fig. 3.11. An RF pulse (length, centre frequency, bandwidth, window...) was designed in Matlab on a personal computer and transfered via ethernet (green line) to a Tektronix AFG 3252 240MHz function generator (FG). The pulse (blue line) is amplified by a Minicircuits ZHL-20W-13 RF power amplifier and excites the pulsing/pitching transducer. A P-I$\mathrm{N}$ diode switch protects the sensitive variable gain amplifier (VGA) from the high voltage pulse and is controlled by the FG (red line). For pulseecho measurements, the small echo signals are amplified by the VGA and recorded on a Yokogawa DL9240L 1.5GHz Oscilloscope, triggered by the FG (magenta line). Pitch-catch measurements make use of the second transducer where the signal is amplified by a Time Gain Compensated VGA (Section 3.3.4) and recorded on the oscilloscope (cyan line). The switch and VGA circuits were located on a custom Printed Circuit Board (PCB) constructed and tested in 2009 prior to this work. External power supplies provided $+12 \mathrm{~V}$ (Agilent E3620A) and $\pm 15 \mathrm{~V}$ (HP E3630A) and GND. Appropriate voltage regulators and decoupling capacitors ensured $\pm 5 \mathrm{~V}$ and $12 \mathrm{~V}$ were available for all components.

The transducers were positioned using Thorlabs LNR50VK3/M XYZ and NR360SP3/M rotational stages with $10 \mu \mathrm{m}$ and $0.01^{\circ}$ step sizes. The stages connected via USB and were controlled through the commercial Thorlabs software 'APTUSER'. These stages allowed the position and aim of each transducer to be controlled with 4 degrees of freedom; X, Y, Z and $\phi$. The $\phi$ corresponded to the pitch rotation of the transducer since the rotational stage was mounted vertically. The precision and accuracy of the measure- 


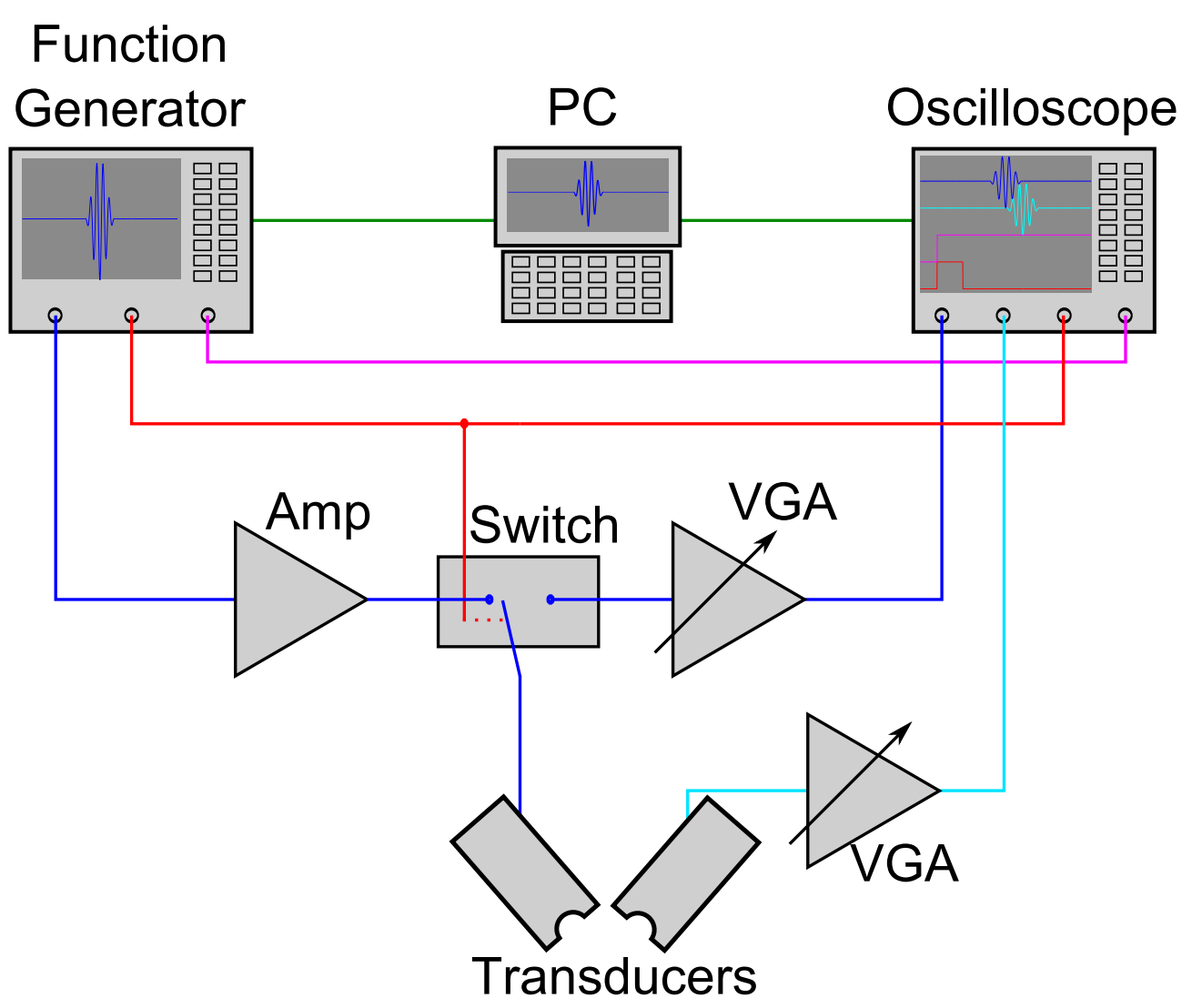

Figure 3.11: Schematic of high frequency ultrasound system illustrating function of electronic equipment and RF signal path.

ments depended on these stages.

\subsubsection{Power Amplifier}

A 20W Minicircuits ZHL-20W-13 power amplifier amplified the pulse from the FG. The amp was chosen for its flat gain (50dB) across a wide-band (10 to $1000 \mathrm{MHz}$ ) that covered the bandwidth of the transducers (10 to $80 \mathrm{MHz}$ ). Input and output impedances were $50 \Omega$ and the amplifier could withstand a full impedance mismatch (short or open) across all phases at the RF output for power outputs not exceeding the maximum [31]. 


\subsubsection{Switch}

A switch was employed to isolate and prevent damage to the separate transmit and receive components. This was achieved by altering the signal path during the two stages of a pulse-echo measurement, seen in Fig. 3.12. The transmit part of the switch (TX SW) was constructed by placing diodes in parallel with their polarities reversed, similar to the 'Expander' circuits in the literature [32]. The high voltage $\left(20 \mathrm{~V}_{\mathrm{pp}}\right)$ excitation pulse alternately forward biased the diodes and enabled transmission of the pulse through to the transducer. The low voltage echo pulse returning from the transducer was below the threshold voltage of the diodes and was rejected. The receive part of the switch (RX SW) was constructed with two Coilcraft WBC4-4L transformers with a 4:1 impedance ratio and two Agilent HMPP-3890 PIN diodes chosen for their short carrier lifetime ( $\tau=200 \mathrm{~ns}$ ). The first transformer was centre tapped to a square wave control signal from the FG. The control signal was timed to be negative during the excitation pulse, resulting in the PIN diodes turning OFF and rejection of the large excitation pulse. This protected the sensitive VGA. The control signal was switched to positive in time for the echo signal. The PIN diodes were turned $\mathrm{ON}$ and the small signal, reduced further by the transformer turns ratio, was allowed to pass as a small pertubation to the DC current, to the second transformer (centre tapped to GND), which restored the small signal to its original amplitude. The balanced nature of the circuit meant largely only the RF was transmitted through the second transformer and on to the VGA.

\subsubsection{Variable Gain Amplifier}

The Variable Gain Amplifier was used to amplify the echo RF signal received from the switch. The amplifier consists of two wide-band (DC to $1000 \mathrm{MHz}$ ) low noise (2.7dB noise figure) fixed gain (25.1dB) Gali-74+ amplifiers from Minicircuits [33] separated by a wide-band (10 to $2000 \mathrm{MHz}$ ) 


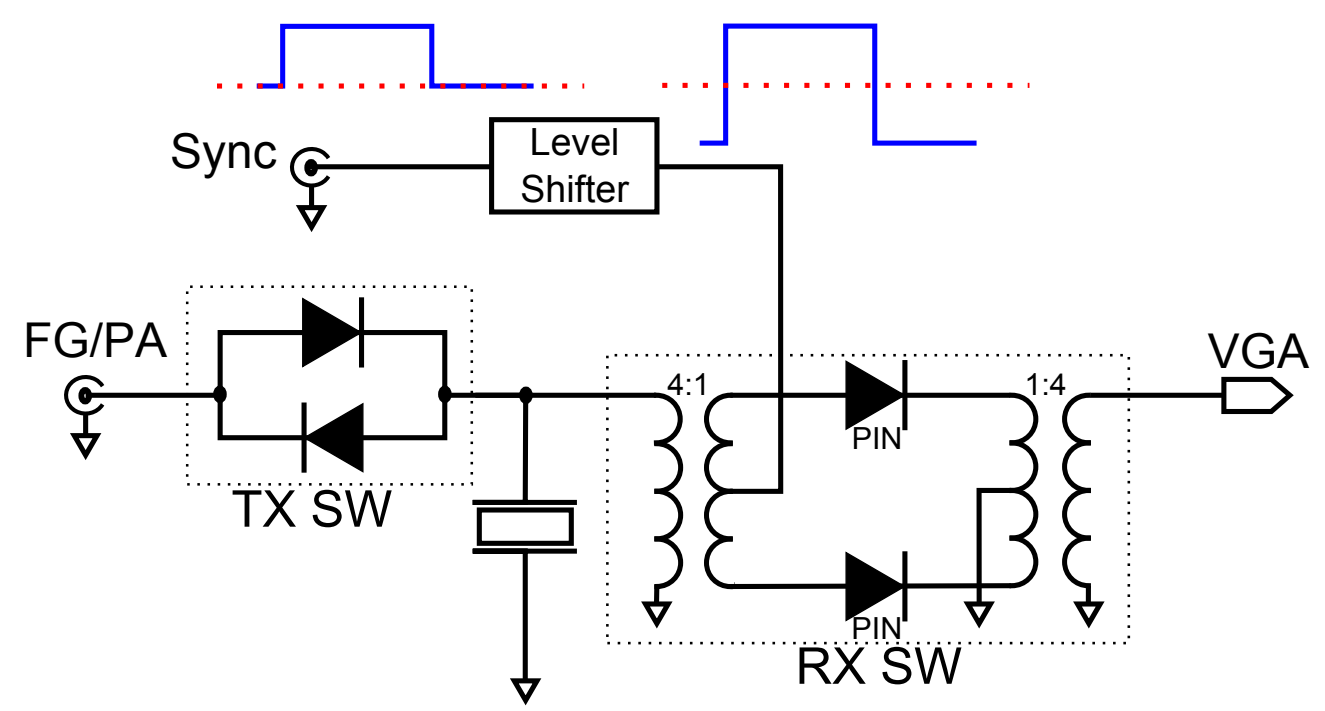

Figure 3.12: Switching electronics employed during a pulse-echo measurements to protect sensitive amplifiers and choose appropriate RF signal path.

MVA-2000+ variable attenuator from Minicircuits [34]. AC coupling was required to prevent the internal DC biasing required by the amplifiers from travelling through the switch's transformer to ground. The amplifiers were powered by DC connected to the RF output pin and required inductors to prevent the RF signal 'escaping' through the power supplies. Both amplifier and attenuator components were matched to $50 \Omega$ input and output impedance. The maximum amplifier output power of 19.2dBm (at $100 \mathrm{MHz}$ ) matched the maximum input power level of the attenuator of $19 \mathrm{dBm}$. The combination of the three components resulted in a variable gain of $45 \mathrm{~dB}$ at $40 \mathrm{MHz}$.

\subsubsection{Time Gain Compensation Variable Gain Amplifier}

Pitch-Catch measurements required an amplifier to raise the level of the signals relative to noise to assist detection. An amplifier with variable gain 
designed, manufactured and tested by other researchers [35, 36, 37] was employed to amplify the signals received at the second transducer. This amplifier possessed a flat gain across a bandwidth of DC to $100 \mathrm{MHz}$ and a variable gain range of $17-65 \mathrm{~dB}$, corresponding to $48 \mathrm{~dB}$ of variable gain. Time Gain Compensation (TGC) was used to further amplify signals that arrive later in time. These later arriving signals had travelled a greater distance and were correspondingly attenuated to a greater extent. To counter this exponential attenuation the amplifier was designed to provide TGC through the use of linear-in-dB VGA components and was triggered by a SYNC function from a FG. Separate potentiometers were used to control the DC and TGC gain, enabling the amplifier to apply all-DC gain, all-TGC gain or a combination of the two. Further details on the operation and construction of this amplifier are available in the literature $[2,35,36,37]$.

\subsection{Signal Processing}

\subsubsection{Matched Filtering}

The time/range resolution of a (single frequency) system is limited by the pulse length, as discussed in Section 3.1. To improve the resolution a pulse of shorter duration (fewer cycles) or a higher frequency must be used. However a reduction in pulse length has a corresponding loss in signal energy which reduces the SNR and broadens the spectrum of the pulse. Increasing the frequency past the specifications of the transducer can also reduce the SNR. To improve the SNR the pulse length or signal amplitude has to be increased, which requires more power from the system. Hence increasing the resolution and SNR simultaneously is a difficult process. A technique to overcome these SNR and resolution constraints is to use matched filtering which is popular in RADAR [19, 38, 39]. Matched filtering involves transmitting a linear frequency modulated (LFM) sinewave or 'chirp' with appropriate windowing. An example for a transducer with 
centre frequency $f_{c}=10 \mathrm{MHz}$ and bandwidth $B=10 \mathrm{MHz}$ is described with reference to Fig. 3.13. The resolution of such a transducer at its highest frequency $(15 \mathrm{MHz})$ in water is $100 \mu \mathrm{m}$. The chirp waveform (blue) has been described [40]

$$
C(t)=\text { window } \times \sin \left(\omega_{a} t+\frac{\pi B t^{2}}{T}\right) \quad 0 \leq t \leq T
$$

where $\omega_{a}=5 \mathrm{MHz}$ is the starting angular frequency and $T=2 \mu \mathrm{s}$ is the length of the chirp. The particular chirp used in this work had a $20 \%$ cosine taper (Tukey) window [29]. The time reversal of the chirp is recorded for reference (green). The received signal (red) consists of two chirps beginning at $0.5 \mu \mathrm{s}$ and $8.5 \mu \mathrm{s}$ that are contaminated with additive white Gaussian noise (AWGN). The received signal is passed through a filter with an impulse response that is the time reversed reference signal i.e. the received signal is convolved with the time reversed reference signal. Hence the filter 'matches' the expected signal. The result of filtering (orange) is two sinc functions centered at $0.5 \mu \mathrm{s}$ and $8.5 \mu \mathrm{s}$. This technique is commonly referred to as 'Pulse Compression' as the filtering compresses the pulses energy to a short period of time. The resulting resolution is inversely proportional to the chirp bandwidth [38]

$$
R=\frac{c}{2 B}
$$

where $c$ is the speed of sound. For the chirp used in this work the resoltuion is $75 \mu \mathrm{m}$, an improvement on $100 \mu \mathrm{m}$. The improvement in SNR is significant. 


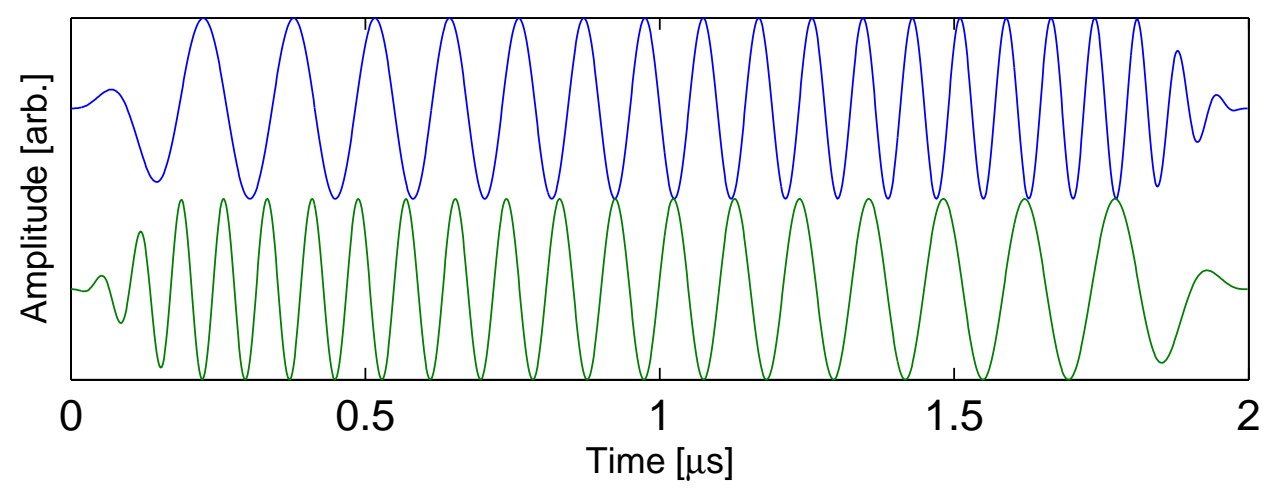

a)

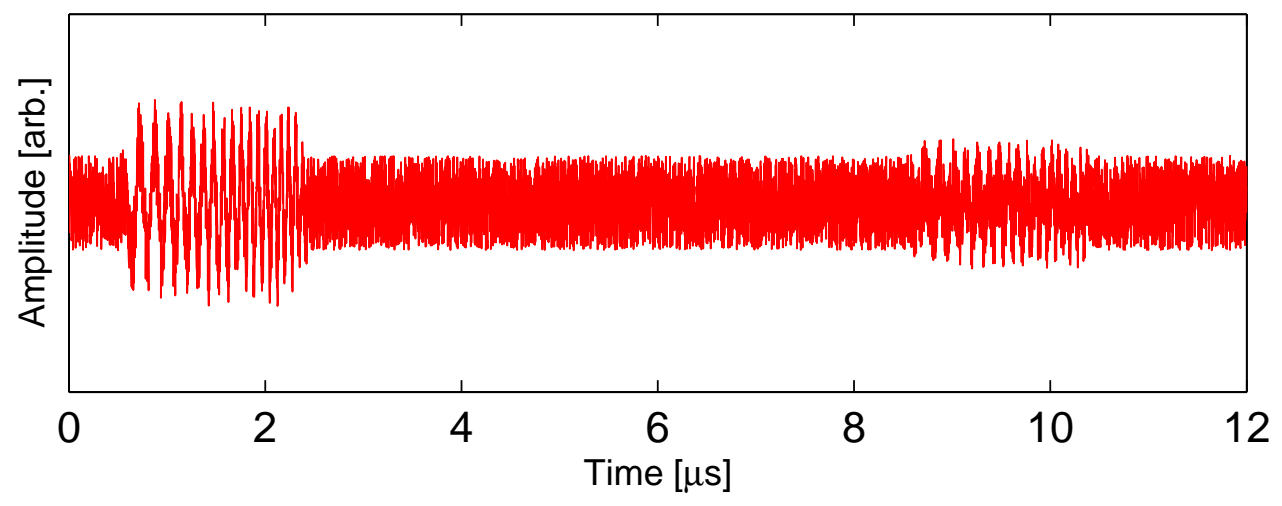

b)

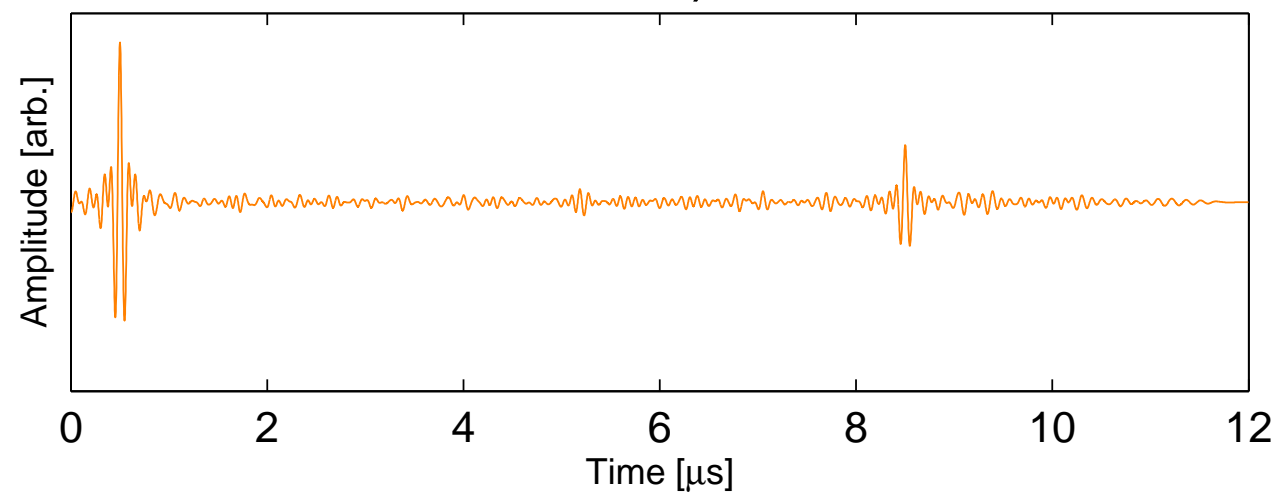

c)

Figure 3.13: Process of Matched Filtering: a) Chirp (blue) and its time reversal (green), b) Typical received signal - two chirps at $0.5 \mu \mathrm{s}$ and $8.5 \mu \mathrm{s}$ buried in noise, c) Result of Matched Filtering - two distinct peaks at $0.5 \mu \mathrm{s}$ and $8.5 \mu$ s. 


\section{Chapter 4}

\section{Coating Thickness Measurements}

This chapter details the investigation into the ultrasonic measurement of coating thickness of two pultruded rods. Any significant variation in the coating thickness of these rods is a potential manufacturing defect and requires a non-destructive testing method for quick identification. The first rod has a pultruded core and a coaxial sheath of extruded plastic approximately $1 \mathrm{~mm}$ thick. The thickness of the sheath was found to vary around the rod, considered an issue of the sheath/core concentricity. The second rod had a pultruded core and paint coating approximately $90 \mu \mathrm{m}$ thick. This investigation employed ultrasonic pulse-echo measurements and time of flight signal processing methods to measure the thickness of the coatings for these rod samples.

\subsection{Sheathed Rod}

The core of the sheathed rod is a white pultrusion of radius $r_{i}=2.25 \mathrm{~mm}$ encased in extruded yellow plastic sheath $1 \mathrm{~mm}$ thick. Ideally the sheath and the core would be coaxial, however a number of samples were found to have manufacturing defects relating to the thickness of the sheath and the corresponding concentricity. A sample was cut in cross section to demonstrate the defect which can be seen in Fig. 4.1. The white core 


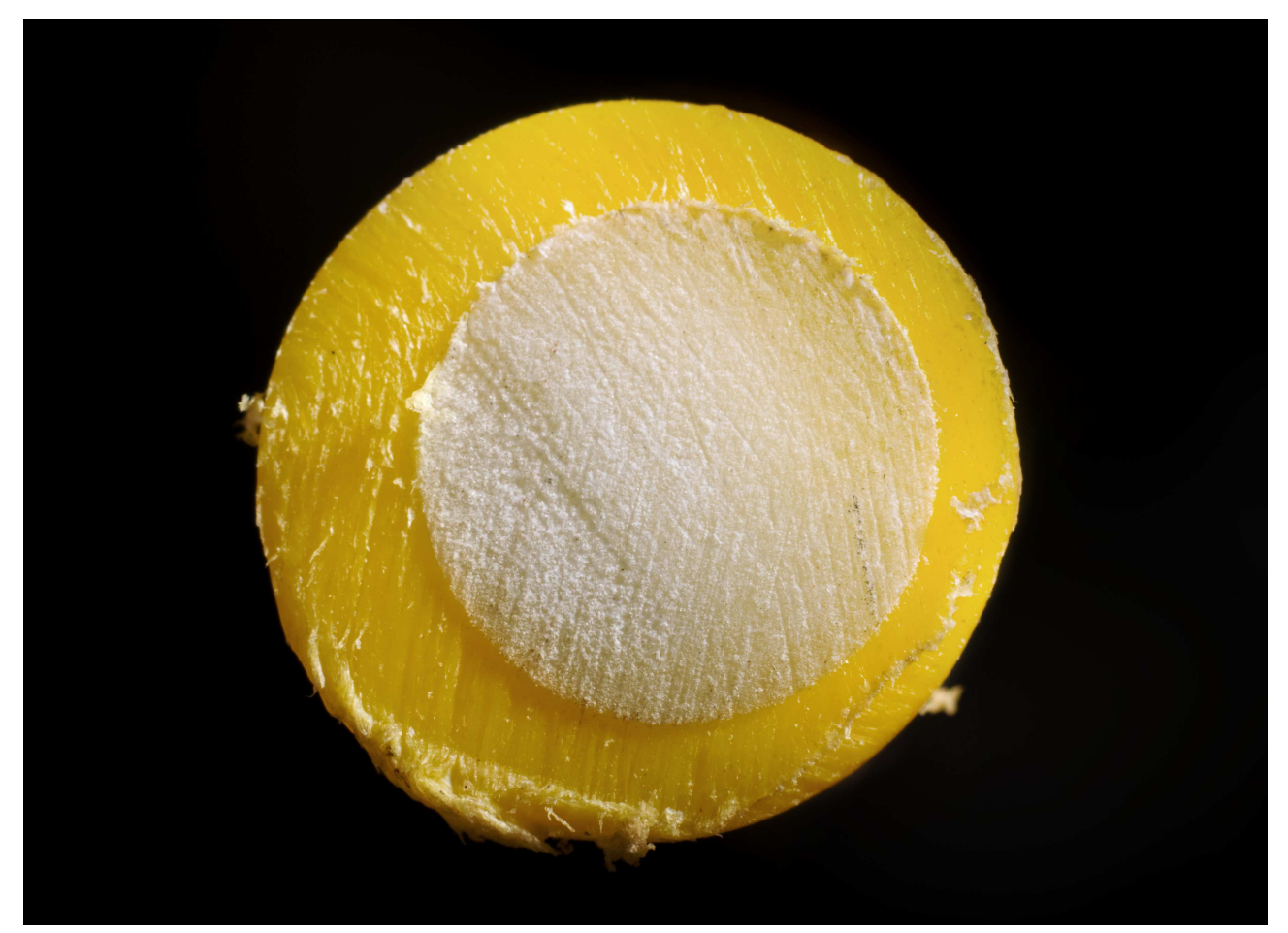

Figure 4.1: Cross-section of a section of the sheathed rod showing the white pultruded core and yellow sheath. A manufacturing defect is the varying thickness of the sheath around the core and the shift of the core to the right. The two rods share a parallel axis however are not coaxial as their axes/centres are not in the same place.

is not concentric with the yellow sheath. The core and sheath share a parallel axis, however the two are not strictly coaxial/concentric as their axes/centres are not in identical locations. An investigation was undertaken into the effectiveness of ultrasound as a non-destructive tool for determining the thickness and concentricity of the sheath. First the likely range of sheath thickness was calculated from the geometry of the rod, followed by a FEA to model an ultrasonic pulse-echo measurement. Finally pulse-echo measurements were made on samples using a $50 \mathrm{MHz}$ transducer. 


\subsubsection{Sheath Thickness Model}

This model provided an indication of the expected variation of sheath thickness for a rod with the given dimensions. The sheath thickness was assigned $\Delta r$ and the angle around the centre axis of the sheath was $\theta$. For any non-concentric rod, the offset of the core centre relative to the sheath centre has a horizontal and vertical component, seen in Fig. 4.2(a). In this diagram the core has shifted 3 (arbitrary) units in the horizontal and 4 units in the vertical direction. The entire rod can be rotated by an angle $\theta_{0}$ such that the offset is simplified to 5 units in the horizontal direction only. This omnidirectional offset of the core centre relative to the sheath centre will be referred to as $d$. This rotation results in a line of symmetry in the horizontal direction, seen as a dotted line. This symmetry enables the range of angles required, $\theta$, to be reduced to $180^{\circ}$. From trigonometry, the sheath thickness $\Delta r$ can be calculated from the following equations

$$
\begin{aligned}
\gamma & =\sin ^{-1}\left(\frac{d}{r_{i}} \sin \left(\theta-\theta_{0}\right)\right) \\
l & =\frac{r_{i}}{\sin \left(\theta-\theta_{0}\right)} \sin \left(180-\left(\theta-\theta_{0}\right)-\gamma\right) \\
\Delta r & =r_{o}-l
\end{aligned}
$$

where $\alpha, \beta, \gamma$ and $\theta$ are the angles defined in Fig. 4.2(b). $r_{i}$ is the radius of the core and $r_{o}$ is the radius of the rod. The variation of sheath thickness with angle is seen in Fig. 4.2(c) for a range of core centre offsets. At one extreme the core and sheath are perfectly concentric, $d=0 \mathrm{~mm}$ (blue) and the thickness remains constant. At the other extreme, $d=1 \mathrm{~mm}$ (magenta), the core will be offset such that the sheath thickness will vary between $0 \mathrm{~mm}$ and $2 \mathrm{~mm}$. 

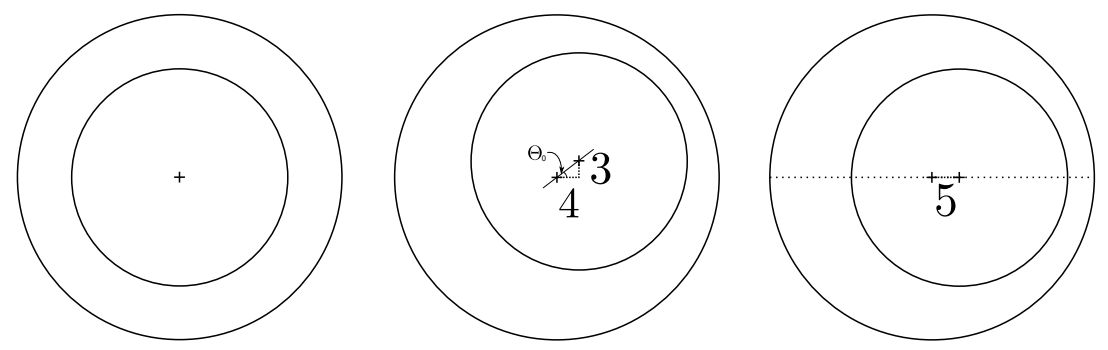

(a)

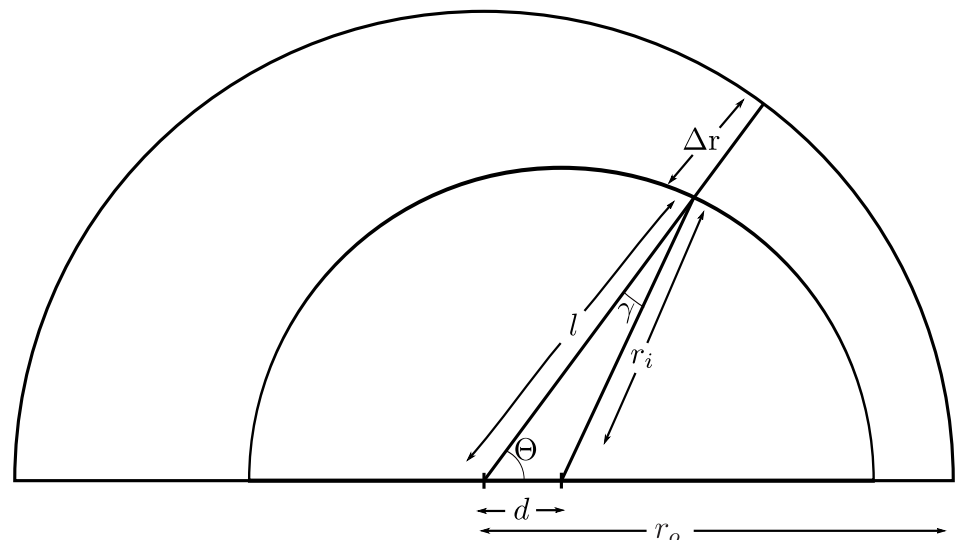

(b)

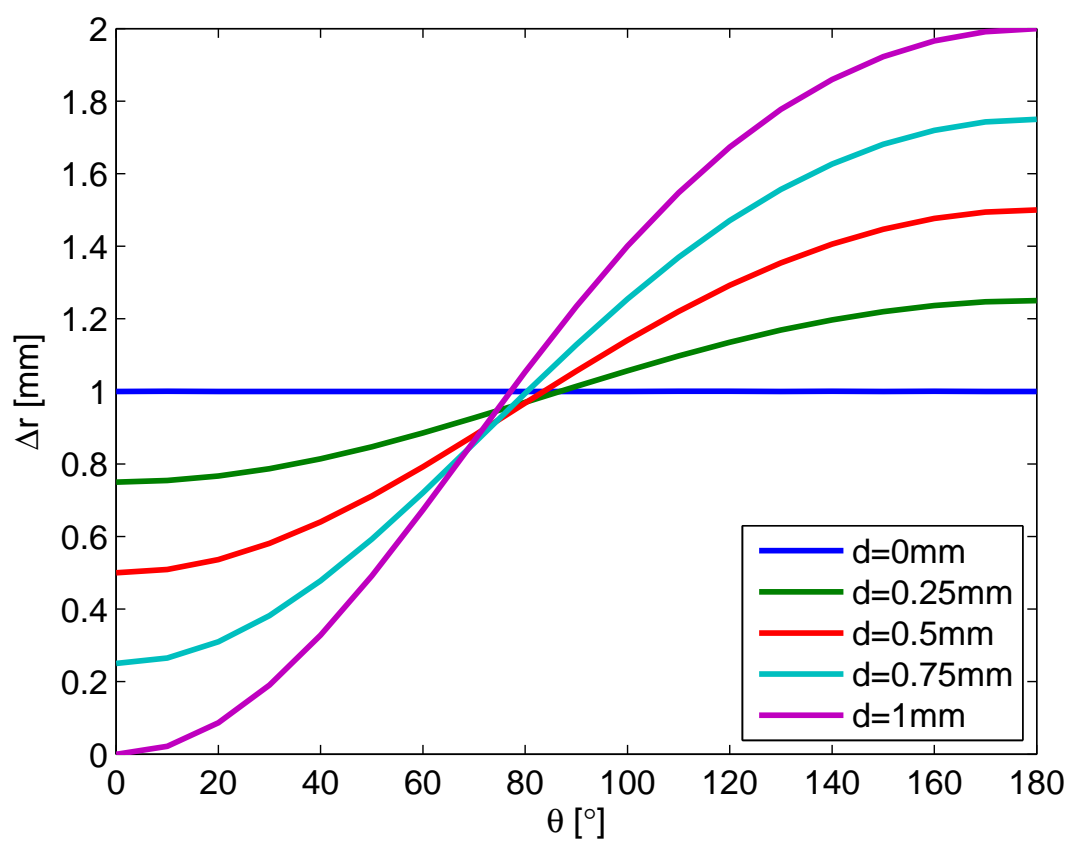

(c)

Figure 4.2: a) Left to right: a concentric rod, two rods with a centre offset of 3 and 4 in the horizontal and vertical directions respectively, and a rod with a centre offset of 5 in the horizontal direction only. b) The angles defined for a rod with a centre offset $d$ that are used to calculate the thickness of the sheath $\Delta r$. c) Ideal curves for $\Delta r$ as $\theta$ varies from 0 deg to $180 \mathrm{deg}$. 


\subsubsection{Finite Element Analysis}

To represent an ultrasonic pulse-echo measurement for determining the thickness of the sheath a model for Finite Element Analysis was created in PZFlex. The model is presented in Fig. 4.3. The model was $400 \mu \mathrm{m}$ high, $2000 \mu \mathrm{m}$ wide and contained two regions of water (blue) with identical properties, defined in Table 4.1. A region of plastic $1 \mathrm{~mm}$ thick (yellow) separated the water from a region of glass (white).

\begin{tabular}{cccc}
\hline & & \multicolumn{2}{c}{ Velocity $\left[\mathrm{ms}^{-1}\right]$} \\
\cline { 3 - 4 } Material & Density $\left[\mathrm{kgm}^{-3}\right]$ & Longitudinal & Shear \\
\hline Water & 1000 & 1480 & - \\
Plastic & 900 & 1950 & 1000 \\
Glass & 3000 & 5800 & 3380 \\
\hline
\end{tabular}

Table 4.1: Properties of Water and Glass

Although the core of the rod was a pultrusion with many glass fibres the macroscopic properties of the core were similar to glass. The top and bottom boundaries were symmetric to ensure no edge effects, whilst the left and right boundaries were absorbing to prevent unwanted reflections from the edge of the model that would not occur in a real experiment. A pulse was generated at the boundary between the two water regions, in place of a transducer. The pulse shape was the second derivative of the Blackman Harris window, with frequency content approximated by a Gaussian at the centre frequency of $10 \mathrm{MHz}$, seen in Fig. 4.4. This pulse is refered to as a 'blak' pulse in PZFlex [41]. This pulse was chosen because there is a distinct peak, helpful for indentification of the pulse's position in time, and the frequency content was well defined.

Snapshots of the simulation are seen in Fig. 4.5. The first snapshot $(0.2 \mu \mathrm{s})$ shows the pulse propagating through the water. In the second snapshot 


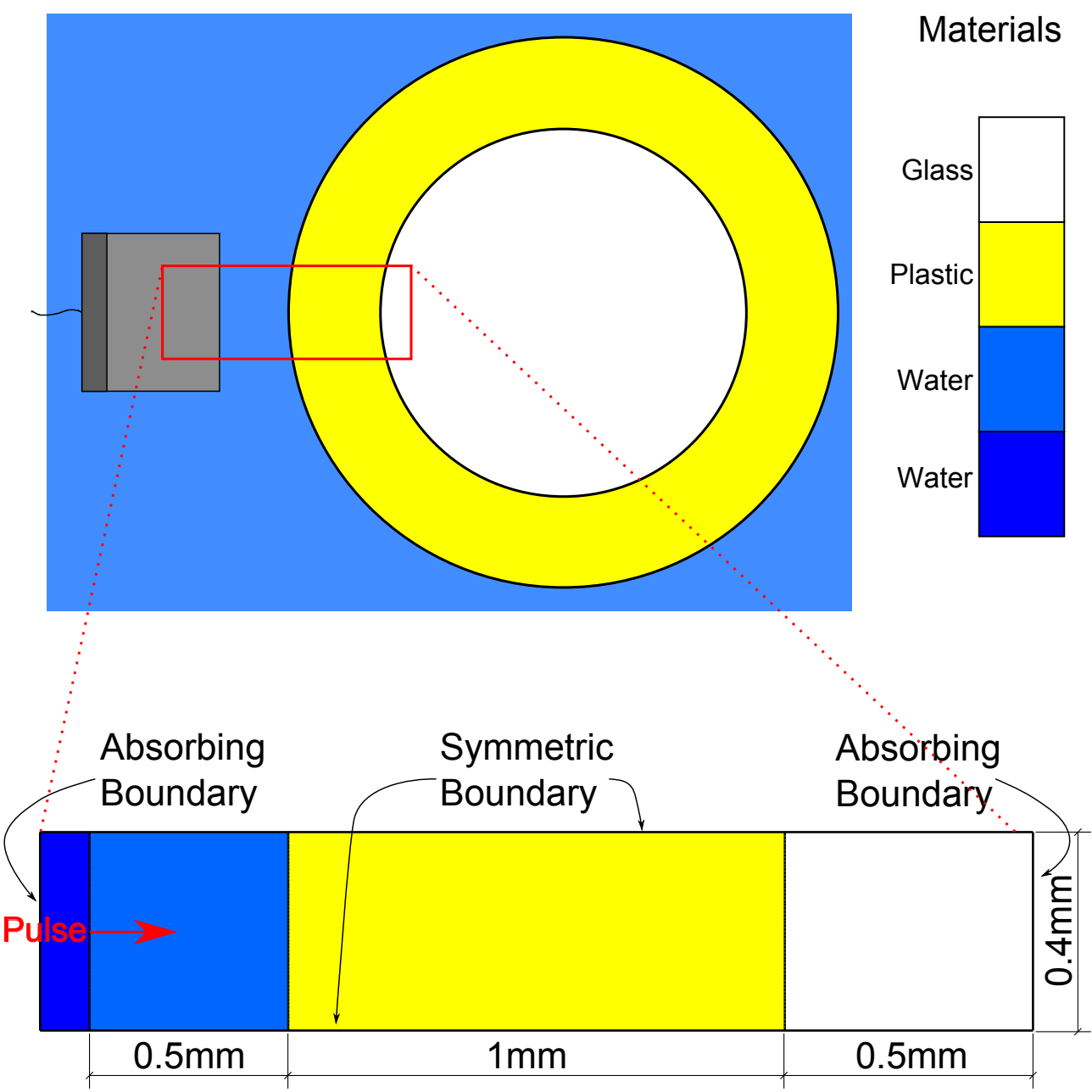

Figure 4.3: The basic sheathed rod model. A transducer is immersed in water in the vicinity of the sheathed rod. The PZFlex model represents the red boxed area: Water, Plastic and Glass.

$(0.5 \mu \mathrm{s})$ the pulse has been partially reflected and transmitted at the waterplastic boundary due to the acoustic impedance mismatch $(\mathrm{Z}=1.5 \mathrm{MRayls}$ vs 2 MRayls). The transmitted pulse propagated through the plastic with a larger wavelength due to the higher acoustic velocity. At the plastic-glass interface the pulse was mostly reflected due to the greater impedance mismatch ( $\mathrm{Z}=2 \mathrm{MRayls}$ vs $4 \mathrm{MRayls})$, seen in the third snapshot $(1.1 \mu \mathrm{s})$. This 

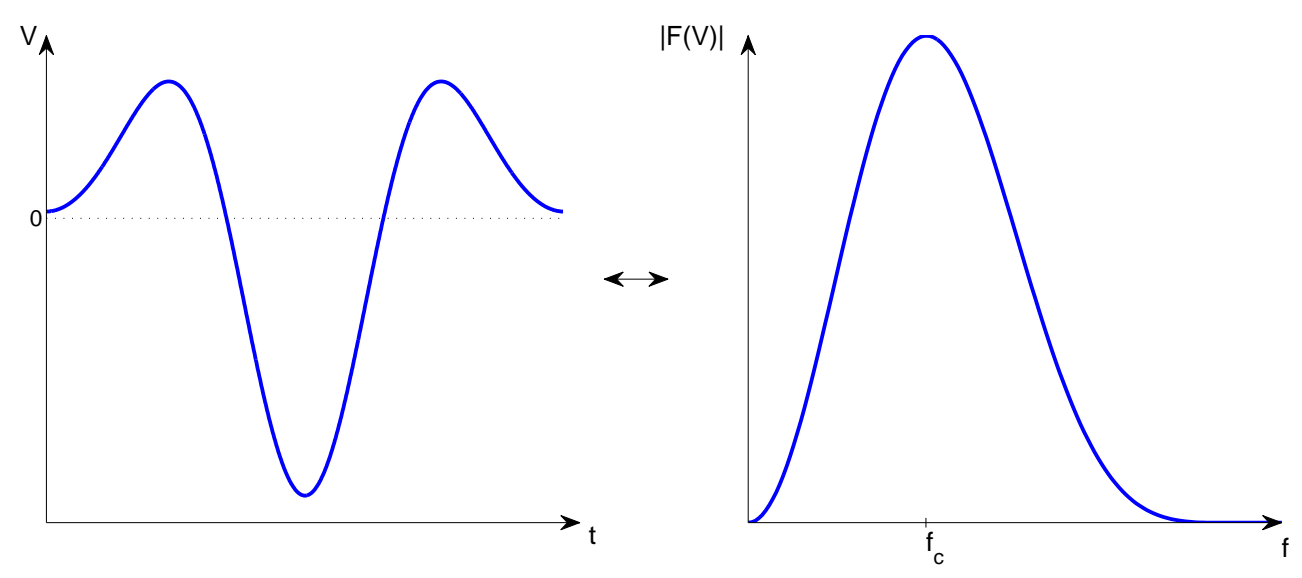

Figure 4.4: A 'blak' pulse, the second derivative of the Blackman Harris window. The frequency content of the pulse is approximated by a Gaussian and is centred at the centre frequency $f_{c}$.

second reflected pulse propagated back through the plastic and was partially reflected and transmitted at the plastic-water boundary, from where the transmitted pulse propagated back to the water-water boundary, seen in the fourth snapshot $(1.5 \mu \mathrm{s})$. The acoustic pressure was recorded for the entire simulation at the water-water boundary and the trace is seen in Fig. 4.6.

The excitation pulse is at $t=0$, the water-plastic reflection at $t \sim 0.6 \mu \mathrm{s}$ and the plastic-glass reflection at $t \sim 1.6 \mu \mathrm{s}$. The two reflection pulses are $180^{\circ}$ out of phase with the transmitted pulse, as expected from theory. The thickness of the plastic was calculated by multiplying the velocity $v=1950 \mathrm{~ms}^{-1}$ by the time between the two reflections $\Delta t=1.0225 \mu \mathrm{s}$ and taking half the value, since the wave travelled the thickness twice. The result was $0.996 \mathrm{~mm}$, in excellent agreement with the value defined in the model of $1 \mathrm{~mm}$ with the error due to the time resolution of the model. The amplitudes of the two pulses are determined by the reflection coefficients at the boundaries hence the second reflection is larger than the first. How- 
ever if the attenuation of the plastic were to be included in the model the second pulse could be smaller than the first, as would be observed in a real experiment. This FEA model fulfilled its purpose in that it demonstrated that a pulse-echo measurement can effectively determine the thickness of a plastic sheath via knowledge of the plastic velocity and the timing of two pulses that reflect due to acoustic impedance differences. 

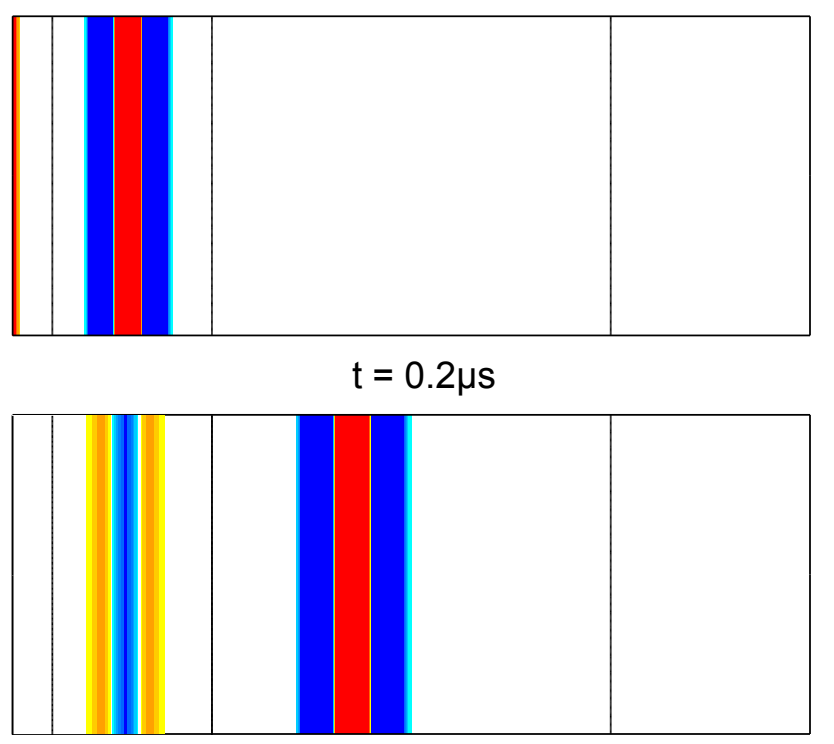

$\mathrm{t}=0.5 \mu \mathrm{s}$

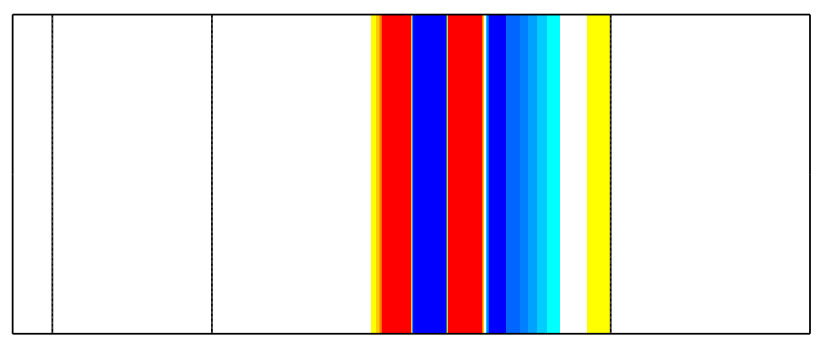

$t=1.1 \mu \mathrm{s}$
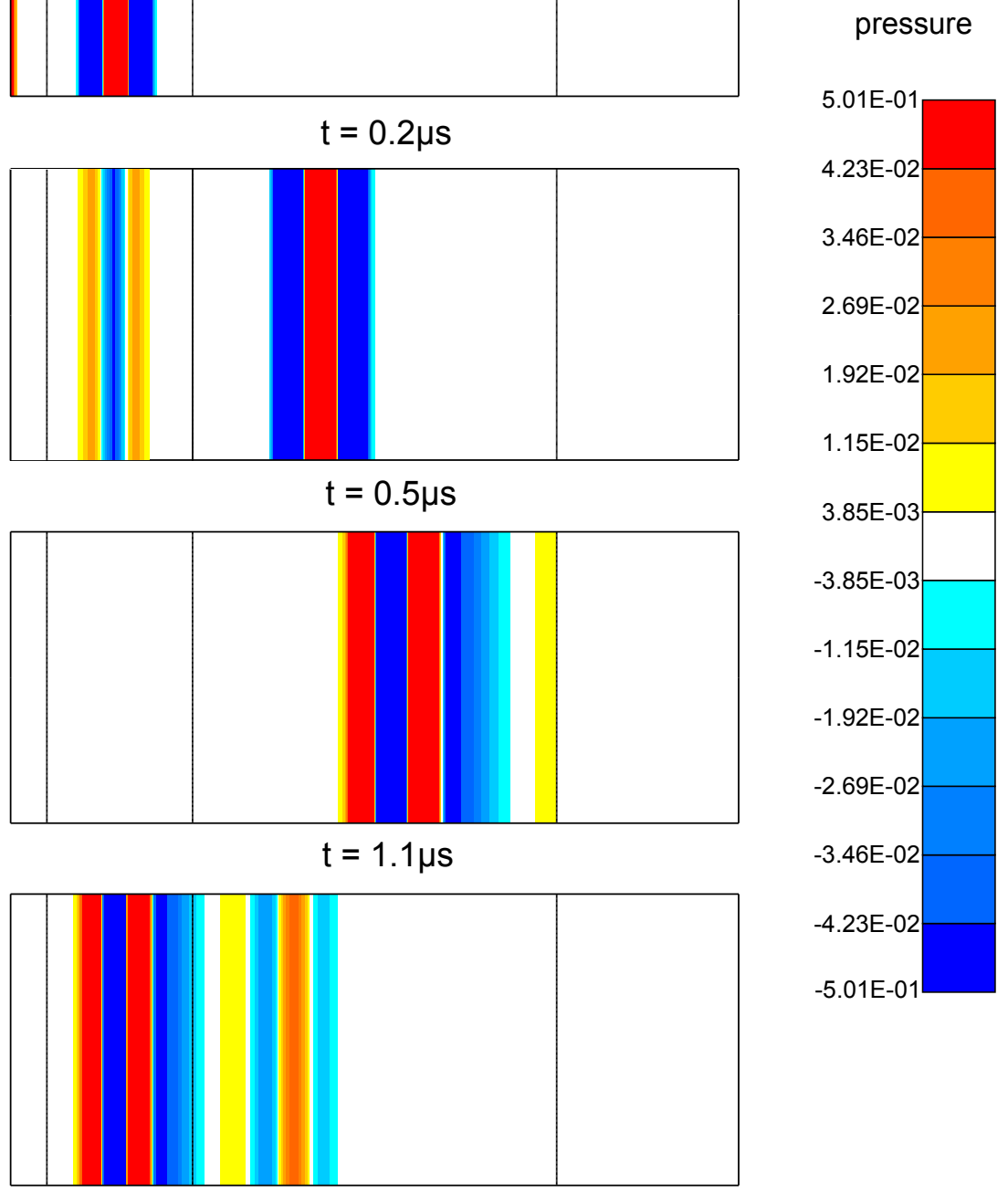

$\mathrm{t}=1.5 \mu \mathrm{s}$

Figure 4.5: Snapshots of the $\mathrm{x}$ displacement. At $t=0.2 \mu$ s the pulse is propagating through water, at $t=0.5 \mu$ s the pulse has partially reflected and transmitted at the water-plastic boundary, at $t=1.1 \mu$ s the pulse has partially reflected from the plastic-glass boundary and at $t=1.5 \mu$ s the pulse is propagating back through the water. 


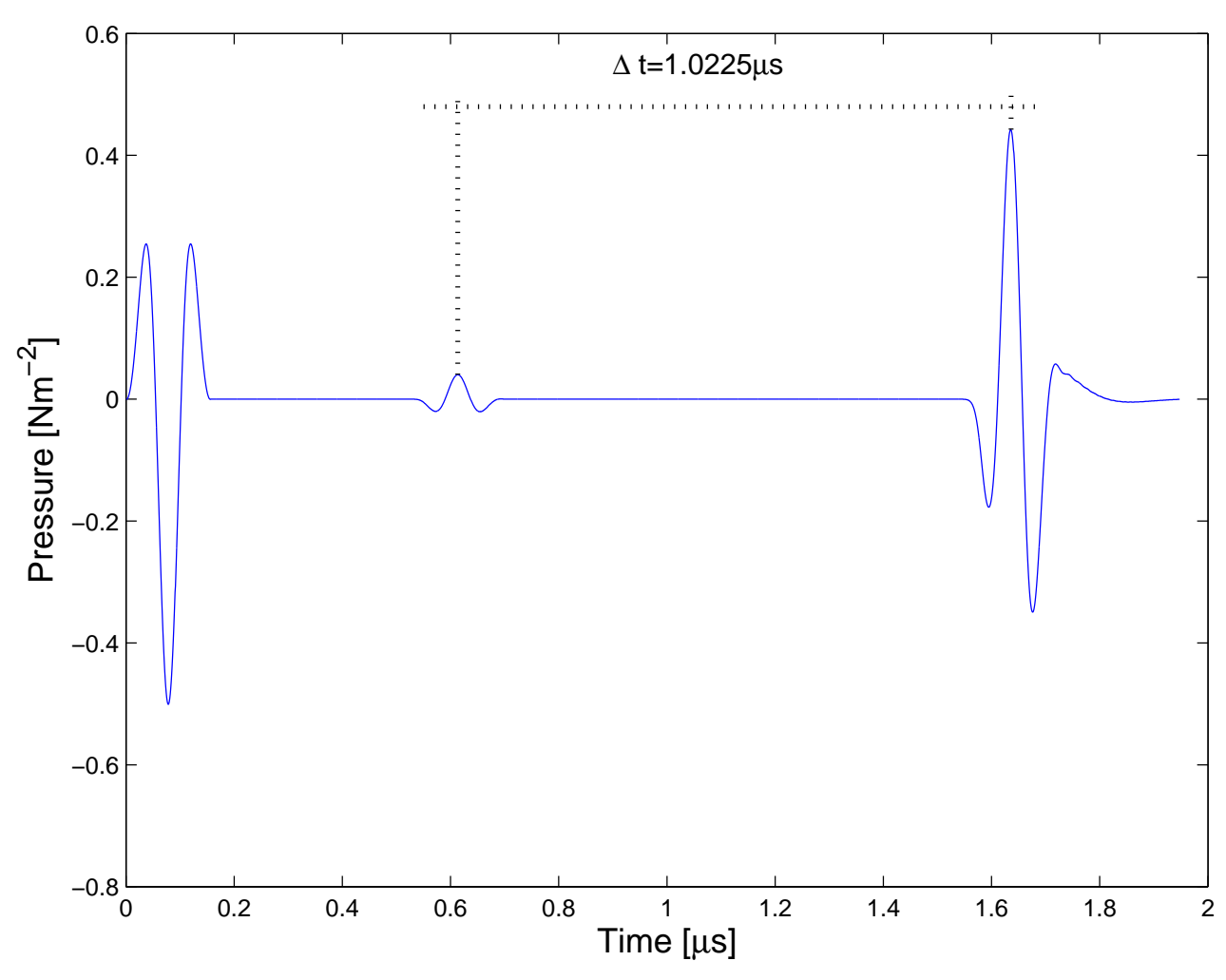

Figure 4.6: Time-Pressure trace for PZFlex model representing a pulseecho model to determine the thickness of plastic. The first pulse is the excitation, the second is the reflection from the water-plastic interface the third is the reflection from the plastic-glass interface. The time delay between the two reflection pulses was $1.0225 \mu \mathrm{s}$ and in plastic $\left(v=1950 \mathrm{~ms}^{-1}\right)$ this corresponds to a thickness of $99.6 \mu \mathrm{m}$, in excellent agreement with the defined thickness of $1 \mathrm{~mm}$. 


\subsubsection{Experimental}

The pulse-echo measurement required a value for the the plastic P-wave velocity, commonly stated as $2000 \mathrm{~ms}^{-1}$ [11]. A small section of plastic measuring $5 \mathrm{~mm} \times 5 \mathrm{~mm} \times 5 \mathrm{~mm}$ was cut from a rod. Using two $50 \mathrm{MHz}$ transducers in a combined pulse-echo/pitch-catch measurement, described in Section 3.2.3, the velocity of the sheath was measured as $2300 \mathrm{~ms}^{-1}$. Immersion transducers were chosen since they provide a uniform acoustic couplant to the cylindrical rod and have a small focussed beam. This small beam size meant the rod surface was approximated as flat. A custom plastic waterbath for these transducers had been constructed previously by other researchers [2]. This waterbath was a cylinder of radius $65 \mathrm{~mm}$ and $55 \mathrm{~mm}$ depth. A circular hole had been cut out of the centre of the base and O-rings fitted such that the bath could be mounted on a cylindrical transducer, with its end face flush with the bottom of the bath, seen in Fig. 4.7(a). This mounting ensured the ultrasonic beam was vertical with respect to the bath and enabled a sample holder to be dipped in and positioned from above the water.

The transducer had an electrically isolated UHF type male connector on its end that was bolted into a custom aluminium block for support. The electrical connection was via a microdot socket mounted on the side of the transducer. The rod was placed in a custom aluminium holder than had two V-shaped prongs. This held the rod horizontal and allowed an ultrasonic beam to impinge on the centre of the rod. The holder was attached to a Thorlabs XYZ stage, enabling the holder and sample to be moved such that the sample was at the focal point of the ultrasonic beam. Since the sheath thickness was to be measured for a full rotation around the rod axis, a protractor system was devised. A small hole $\phi=1 \mathrm{~mm}$ was drilled across the rod and a thin piece of aluminium pushed through the hole to act as a marker. A clear piece of plastic was attached to the sample holder with $10^{\circ}$ increments marked around the centre of the rod, seen in Fig. 4.7(b). This 
provided a crude measurement of the angle that the rod had rotated, with an error of $\sim \pm 3^{\circ}$. Although this error was relatively large, it was acceptable as only a rough indication of the angle was required for this proof of concept measurement. The pulse used for initial measurements was a 5 cycle cosine centred at $25 \mathrm{MHz}$ with a Hann window to ensure a distinct peak and narrow frequency band. This frequency was chosen because it was within the bandwidth of the transducer and ensured the frequency dependent attenuation through water was reduced. As a result the length of the pulse was $200 \mathrm{~ns}$ and the resolution reduced to $200 \mu \mathrm{m}$. This was adequate because the first sample tested was close to an ideal concentric rod so the measurement was not near this resolution limit. A typical oscilloscope trace for a pulse-echo measurement is seen in Fig. 4.8.

The first noteable feature is the three voltage spikes at $\sim 10 \mu \mathrm{s}$, the first and third are due to the control signal for the PIN diode switch, while the second is the excitation pulse from the FG. There are pulses at $\sim 30,50,70$ and $90 \mu$ s which are due to $19 \mu$ s delay line reflections in the transducer. These pulses are ignored as they contain no data about the sample. The pulses of interest are at $\sim 47 \mu$ s and are shown surround by a red box. These pulses are from the sample and have been gated, seen in the two bottom traces for sample orientations of $0^{\circ}$ and $270^{\circ}$. In the $0^{\circ}$ trace there are two pulses are separated by $\sim 0.5 \mu \mathrm{s}$, corresponding to a sheath thickness of $0.5 \mathrm{~mm}$. The SNR of the second pulse was $37 \mathrm{~dB}$ enabling the pulse to be easily identified. Turning to the $270^{\circ}$ trace, the pulses are separated by $\sim 1.15 \mu \mathrm{s}$, corresponding to $1.15 \mathrm{~mm}$. The SNR for this second pulse (at $t=47.8 \mu \mathrm{s}$ was only $12 \mathrm{~dB}$ due to the attentuation from travelling further in the plastic. This pulse had the smallest SNR for the entire measurement. A script in Matlab combined the traces for all 36 measurements and graphed the calculated sheath thickness $\Delta r$, seen in Fig. 4.9(a). A Least Squares fit of the data to (4.1) was completed using the 'lsqcurvefit' algorithm and this fit is displayed as a red dotted line. The fit determined the shift of 
the core centre $d$ and the rotation required to find the line of symmetry $\theta_{0}$ described in Section 4.1.1. In Fig. 4.9(b) the data is presented as a cross section of a rod, with the black line defining the sheath and magenta the line of symmetry. This cross section provides a useful visualisation of the accuracy of the measurement. 


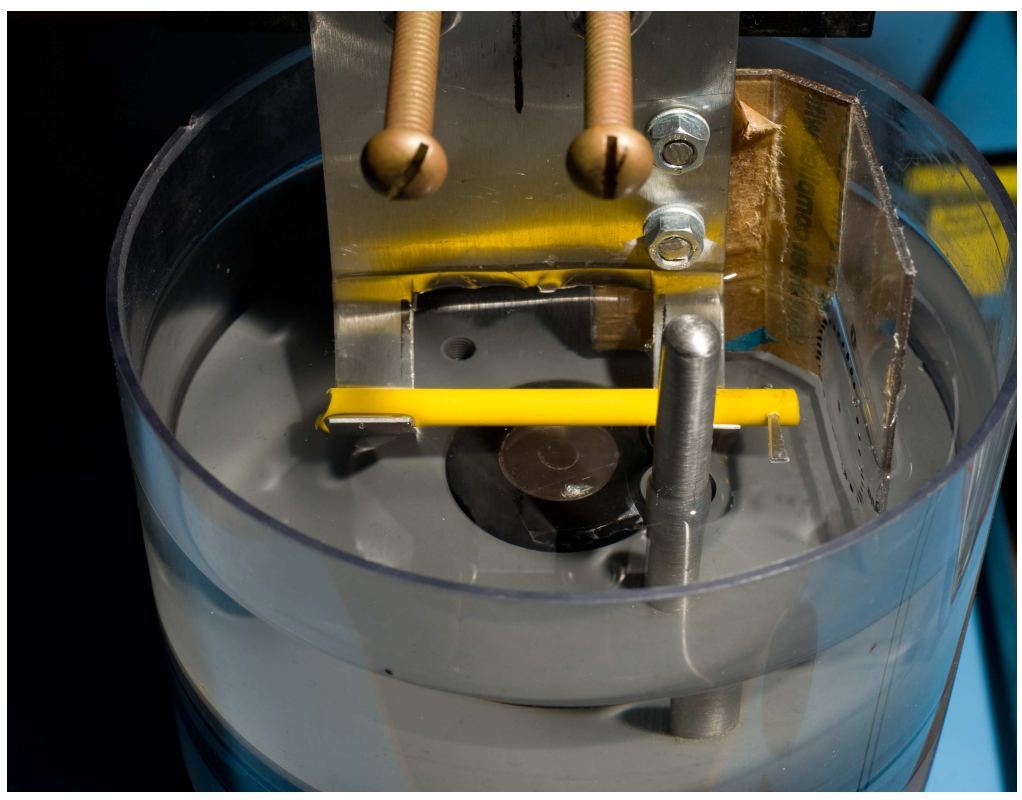

(a) Sheathed rod sitting on custom aluminium holder in custom water bath. The transducer face is the clear circle inside the black ring in the centre of the photo. The lens is the clear circle in the centre of the transducer face.

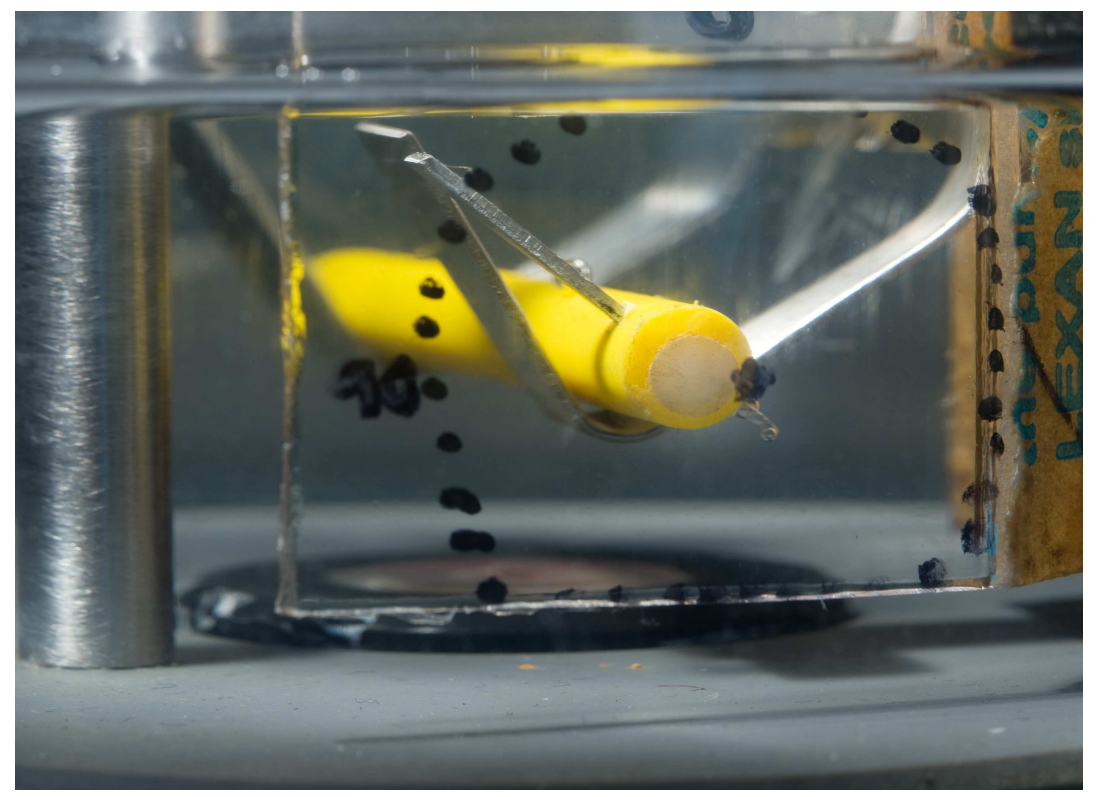

(b) Custom protractor in water bath for measuring the angle of the sheathed rod

Figure 4.7: Photographs of immersion setup for measuring thickenss of sheathed rod. 
Typical Oscilloscope Trace for Sheathed Rod
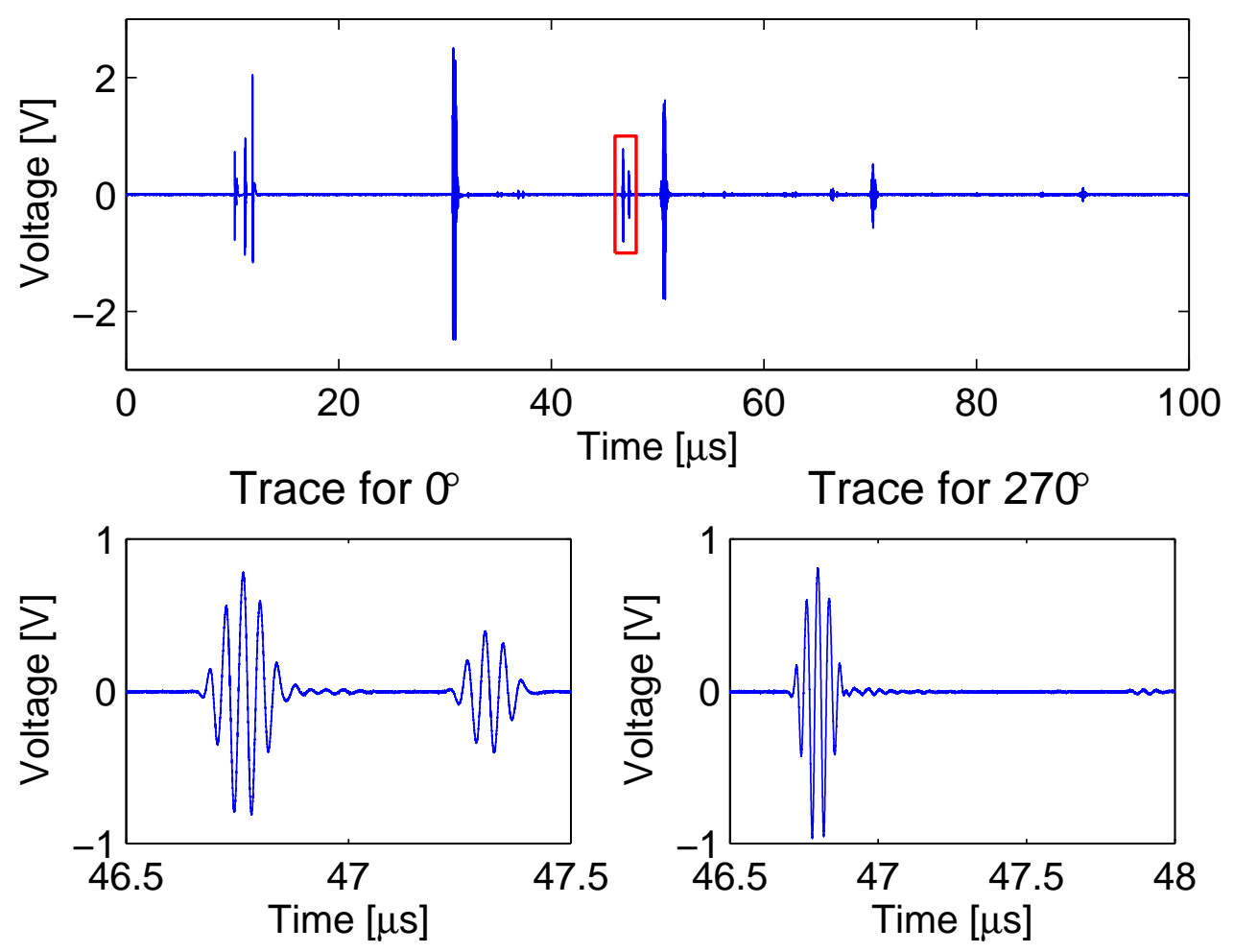

Figure 4.8: Typical oscilloscope traces for a measurement of the sheath thickness. The top trace shows the entire record for a pulse-echo measurement. At $10 \mu$ s there are three pulses, the first and third are from the control signal for the PIN diode switch while the second is the pulse from the function generator. Delay line reflections are seen at $\sim 30,50,70$ and $90 \mu \mathrm{s}$ and can be ignored. The pulses of interest are in the red box at $\sim 47 \mu$ s and are reflections off the sample. These signals are shown in greater detail for the rod orientated at $0^{\circ}$ and $270^{\circ}$. At $0^{\circ}$ the second signal (from the core) is strong with a high SNR $(37 \mathrm{~dB})$. However at $270^{\circ}$ the SNR of the signal from the core is much less $(12 \mathrm{~dB})$ and is hard to distinguish at the given scale at $t=47.8 \mu \mathrm{s}$. The small signal is due to attenuation of the wave that travelled a greater distance through the plastic. 


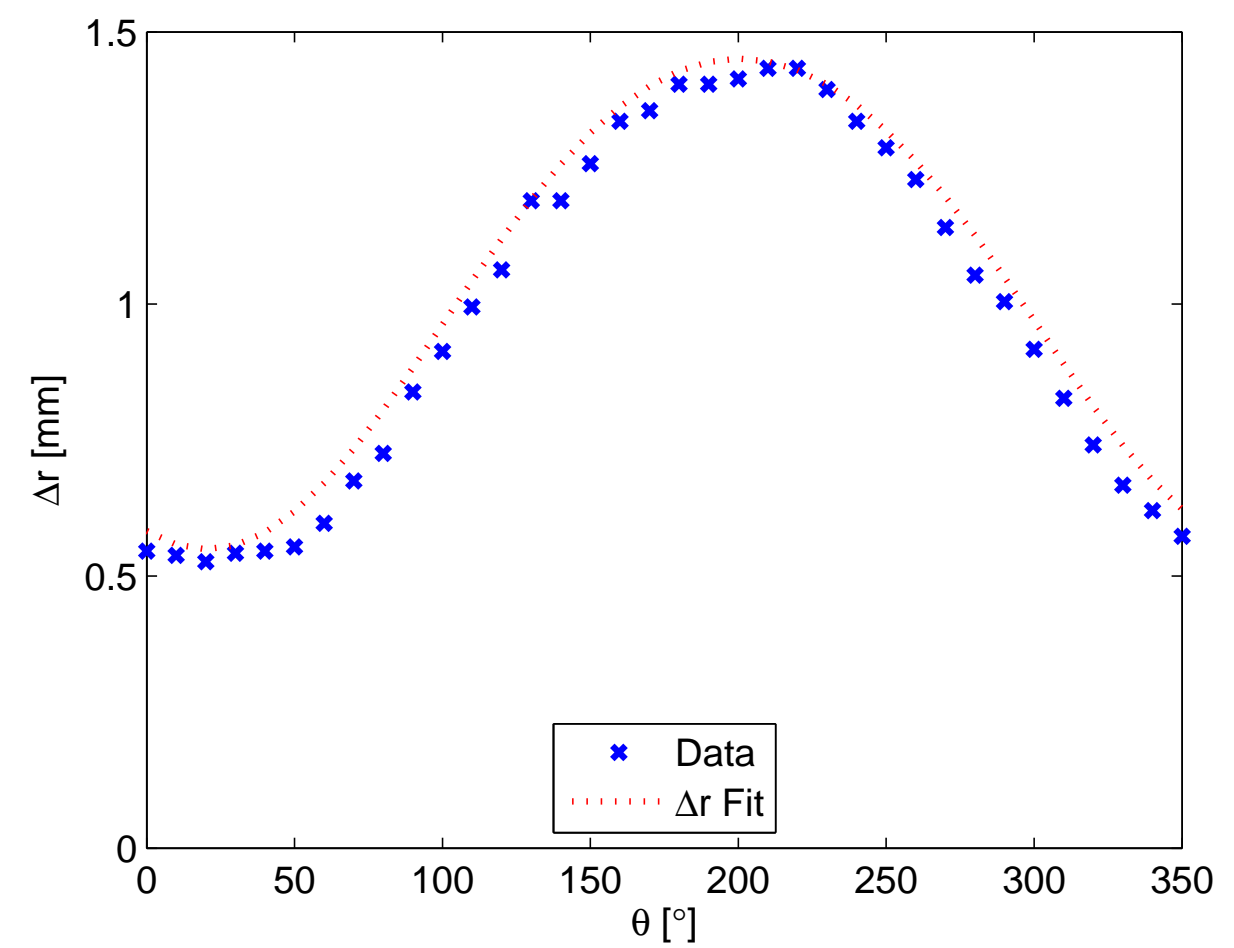

(a) Sheath thickness $\Delta \mathrm{r}$ for a full rotation around the sample - blue crosses. Dotted red line represents an ideal fit.

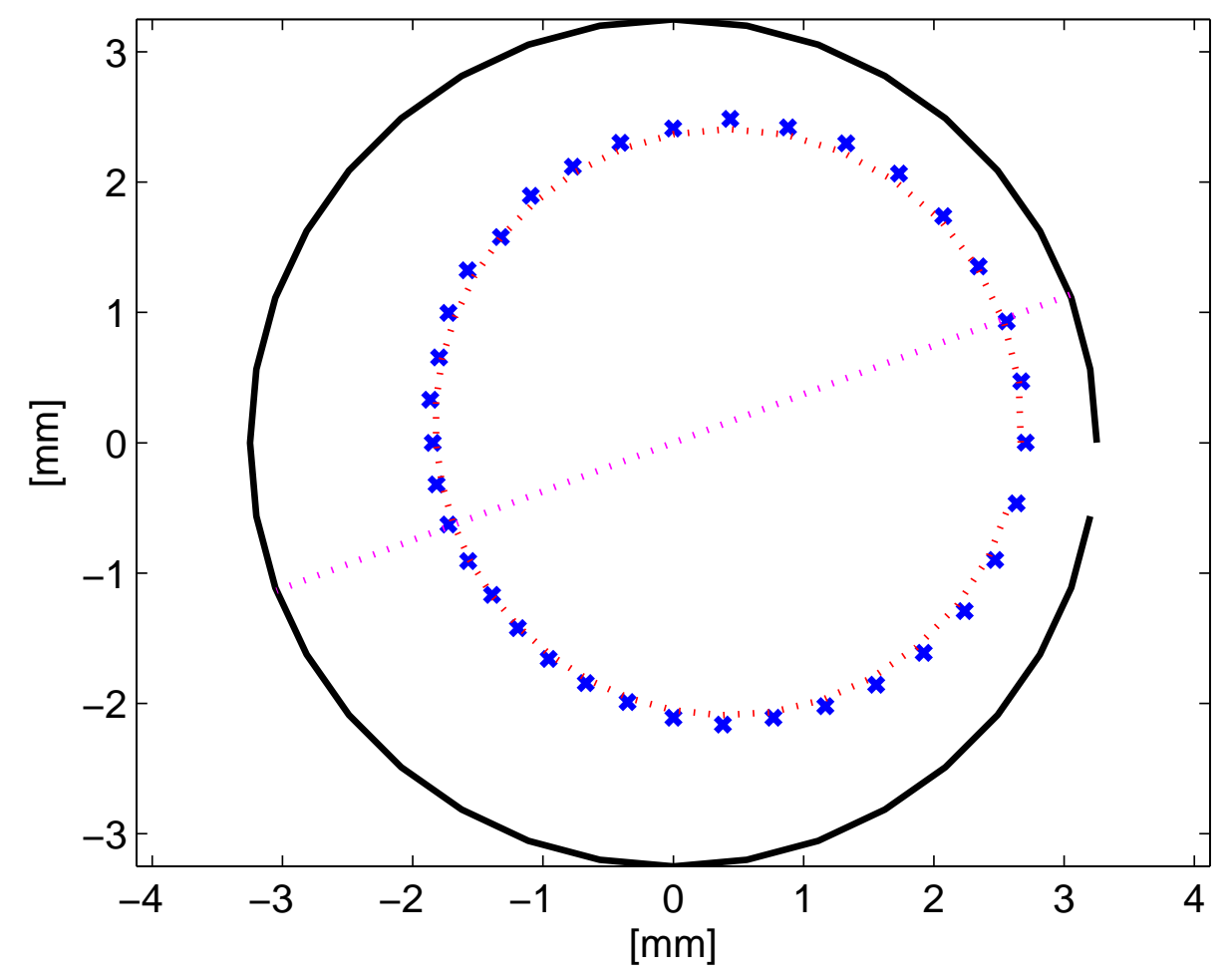

(b) Measurement presented as a cross section.

Figure 4.9: Experimental sheath thickness measurement results. 


\subsection{Painted Rod}

The second rod investigated had a pultruded core $10.00 \mathrm{~mm}$ in diameter coated in a thin layer of paint between $80 \mu \mathrm{m}$ and $100 \mu \mathrm{m}$ thick [5]. To calibrate for the paint properties a $5 \mathrm{~mm}$ square sample of paint was cut and peeled from a rod. A combination pulse-echo/pitch-catch ultrasonic measurement at $25 \mathrm{MHz}$ was used to simultaneously determine the velocity and thickness of the sample, measured as $2000 \mathrm{~ms}^{-1}$ and $160 \mu \mathrm{m}$ respectively. This sample was too thin to be measured accurately with calipers.

The expected thickness of $\sim 90 \mu \mathrm{m}$ influenced the minimum frequency required to resolve the paint thickness in an ultrasonic pulse-echo measurement. Since the paint thickness was to be measured using the $50 \mathrm{MHz}$ transducers the pulse had to be between $25 \mathrm{MHz}$ and $60 \mathrm{MHz}$. To achieve high resolution a high frequency must be used, hence the highest resolution for a single cycle pulse at $60 \mathrm{MHz}$ would be $17 \mathrm{~ns}$. However due to the preference to use a 5 cycle pulse for improved SNR, the resolution would deteriorate to $84 \mathrm{~ns}$, close to the expected thickness of $90 \mu \mathrm{m}$. The scattering from the fibres on the surface of the core, attenuation through water at this high frequency and transducer impulse response would significanly reduce the amplitude of the signal received in a pulse-echo measurement. To counter these effects matched filtering was chosen to achieve the desired resolution.

An investigation into the abilities of the matched filter was conducted. A chirp was created in Matlab with a bandwidth from $30 \mathrm{MHz}$ to $60 \mathrm{MHz}$ lasting 200ns with a Tukey window (coefficient =0.2), seen in Fig. 4.10 as the blue line. This chirp excited a $50 \mathrm{MHz}$ transducer in a pulse-echo measurement that reflected the pulse off a flat sample of glass. The oscilloscope trace was recorded and gated to isolate the reflection pulse, seen in Fig. 4.10 as the green line. 

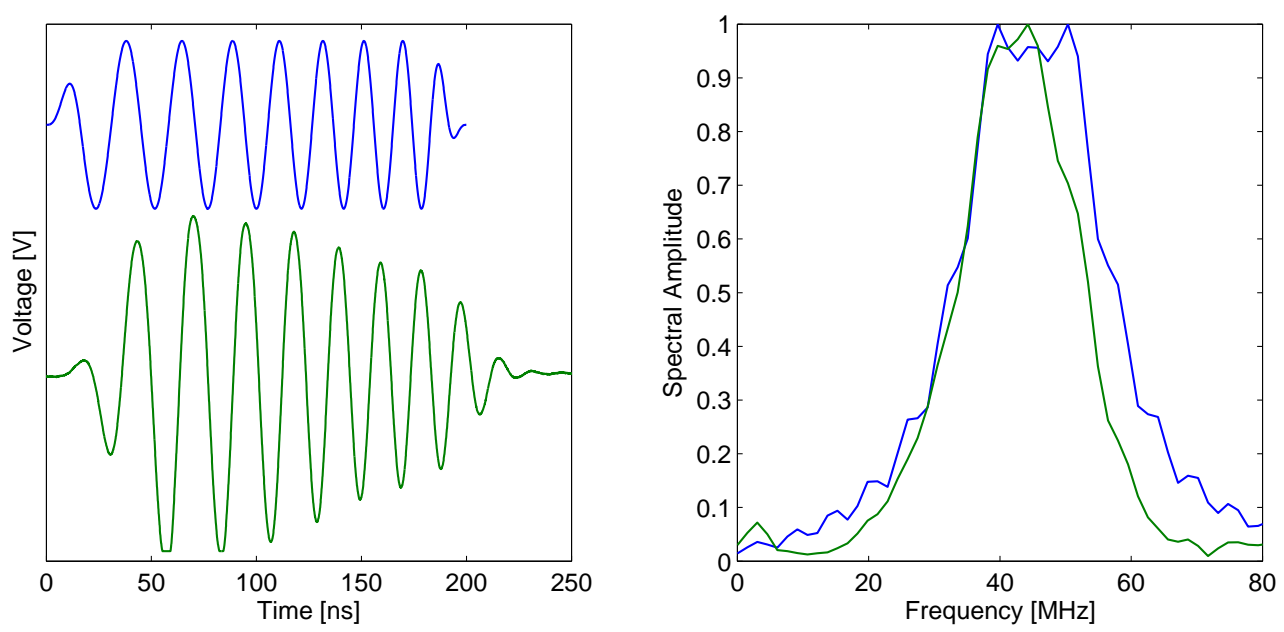

Figure 4.10: Waveform and spectral components of excitation chirp pulse (blue) (Properties: $\mathrm{T}=200 \mathrm{~ns}, \Delta f=30 \mathrm{MHz} \rightarrow 60 \mathrm{MHz}$, Tukey window coefficient $=0.2$ ) and recorded pulse (green), reflected off glass block immersed in water.

The first noticeable difference between the two chirps is the frequency content. This is explained by the attenuation of higher frequencies, seen in the spectral amplitudes, due to the water path and the impulse response of the transducer (convolving with the voltage pulse while transmitting and the pressure pulse while receiving). The functionality of the matched filter and peak detection algorithm that were developed in Matlab are described with reference to Fig. 4.11.

Two chirp pulses arrive at the transducer and are overlapped by $34 \mathrm{~ns}$, the second is one third the amplitude of the first, seen in a). The two pulses (blue and red) are summed with AWGN to represent a real system, seen in b). A matched filter is applied to the observed pulse, with the impulse response the time reversal of one of the chirps. The output of the matched filter is seen in c) with the peak identified by a red cross. This peak cor- 

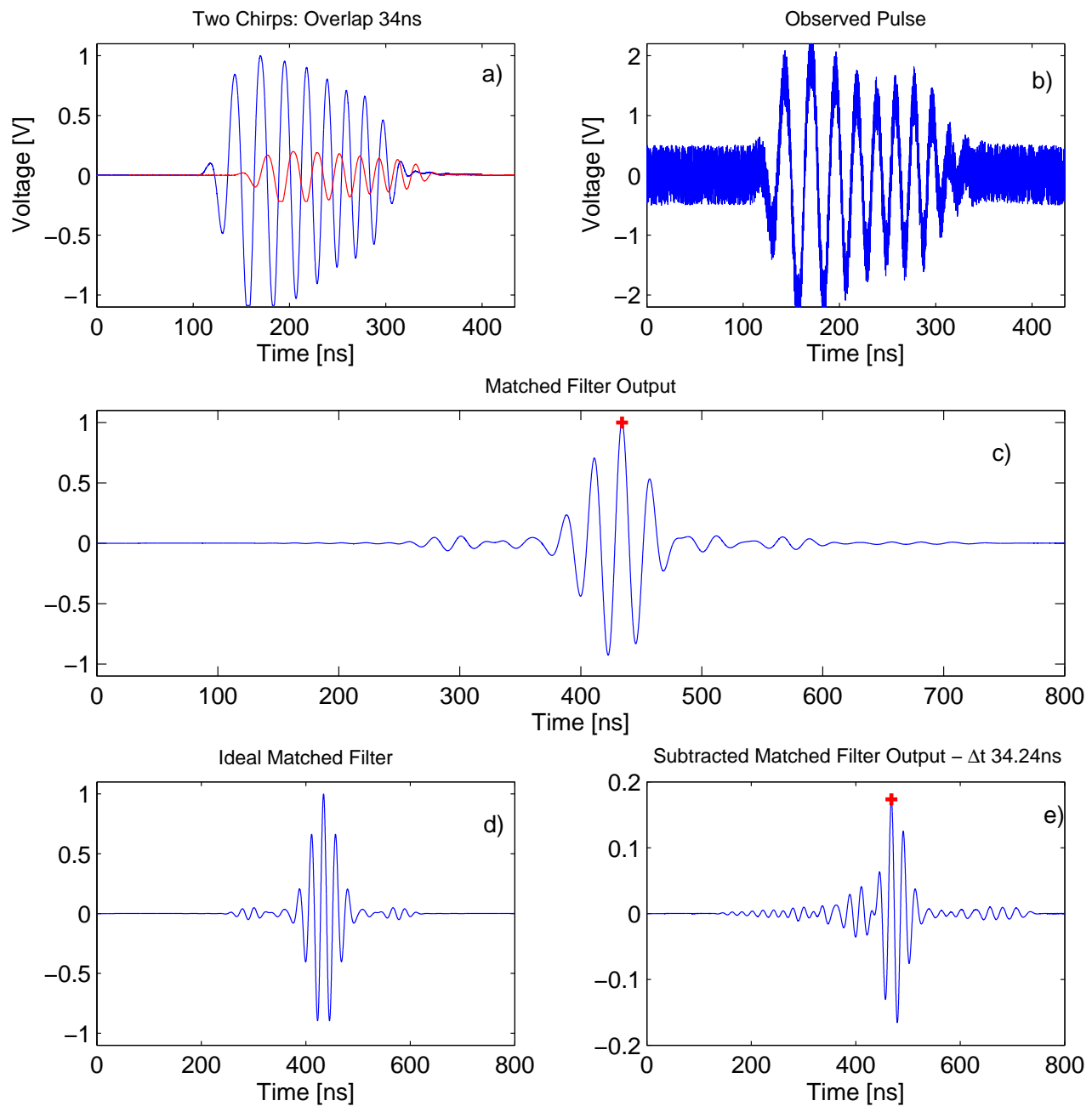

Figure 4.11: Two overlapping pulses, in a), are summed with AWGN to simluate the pulse observed on an oscilloscope, displayed in b). A matched filter, with impulse response the time reversal of one of the chirps, produces a pulse with a single peak that is detected, displayed in c), that corresponds to the first chirp. The output of the matched filter to a single chirp, d), is subtracted from c) to produced e), from which a second peak corresponding to the second chirp, is detected. The time difference between the two peaks is calculated and is in excellent agreement with the defined overlap. 
responds to the first (blue) pulse. The peak for the second pulse is not observed. This is due to the two pulses overlapping. The method described in Section 3.4.1 did not account for overlapping pulses. To separate the overlapping pulses, the output of an ideal matched filter - the output from a single chirp with the filter, displayed in $\mathrm{d}$ ) - is subtracted from the matched filter output. The result is displayed in e). The peak of the subtracted matched filter output is found, which corresponds to the second (red) pulse. The time difference between the two peaks was calculated as $34.24 \mathrm{~ns}$, in excellent agreement with the defined overlap of $34 \mathrm{~ns}$.

One advantage of this signal processing process is the reduction of noise by the matched filter achieved because the noise is random and not correlated to the filter. The signal of interest could be completely buried in noise and still be detected due to the random nature of the noise. Another advantage is the second chirp can be significantly smaller than the first and it will still have a distinct matched filter response and corresponding peak that can be detected. To test the resolution limit of the chirp and signal processing system the overlap of the two chirps was varied from 10ns to $150 \mathrm{~ns}$. The result of the process is seen in Fig. 4.12.

For overlap times greater than 19ns the calculated time is in excellent agreement with the defined time with an average error of $0.3 \mathrm{~ns}$. Below 19 ns the error of the calculated time exceeds $5 \%$ of the defined time which is expected since the highest resolution was calculated as $17 \mathrm{~ns}$, the difference being the small attenuation of higher frequencies identified above. The chirp and signal processing used provide the required resolution to detect paint thicknesses less than the expected value of $80 \mu \mathrm{m}$. A pulseecho measurement was performed on a rod from which the paint thickness was calculated as $97.92 \mu \mathrm{m}$, seen in Fig. 4.13 .

To determine the systematic error of the process ten measurements were 


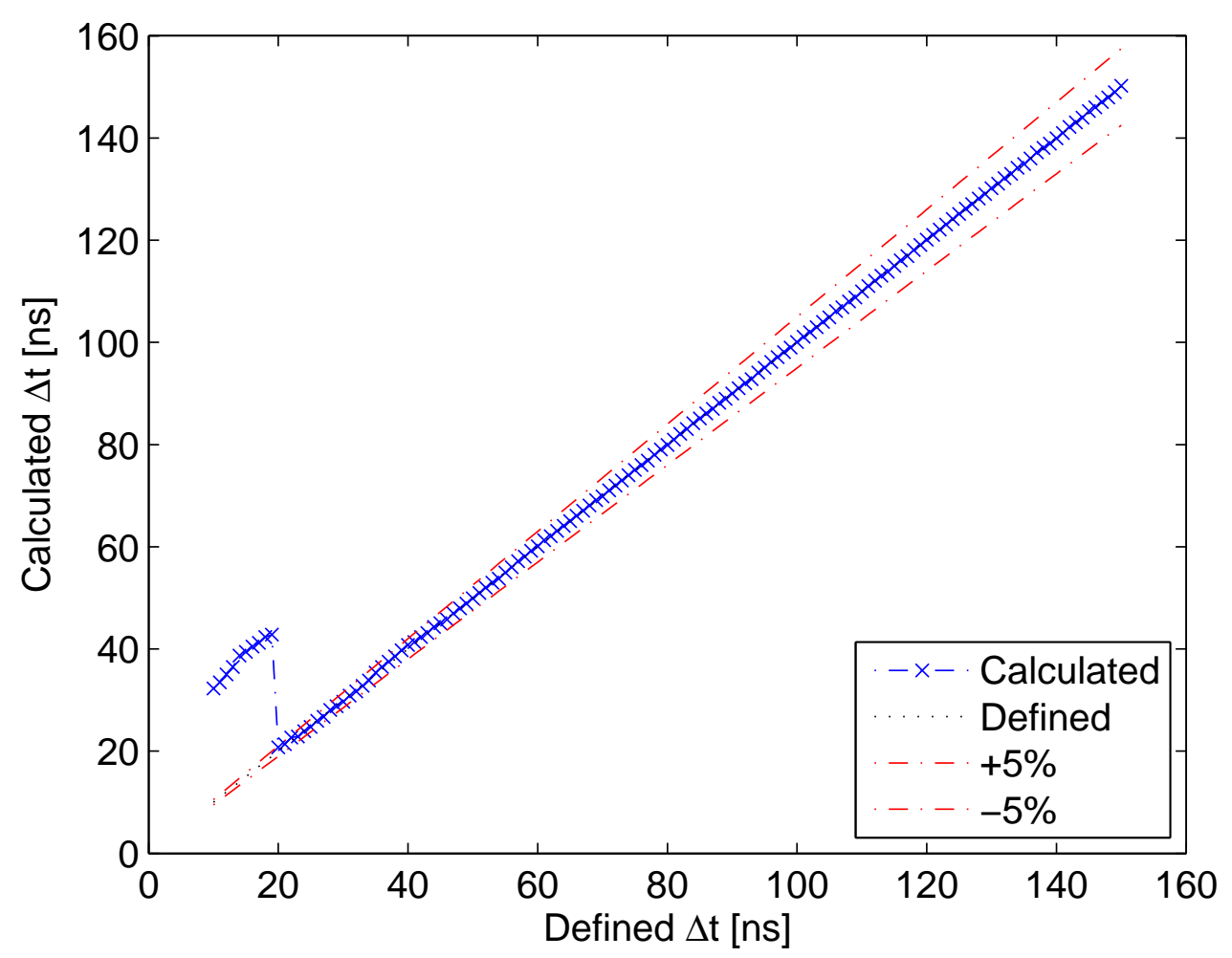

Figure 4.12: Output of matched filter when detecting two chirp pulses that are overlapping by a defined $\Delta t$. The accuracy is excellent for overlaps greater than 19ns, as predicted from theory.

made without moving the rod. The result remained at $97.92 \mu \mathrm{m}$ with an error of $\pm 0.04 \mu \mathrm{m}$, which corresponds to the sampling rate of the oscilloscope $(2.5 \mathrm{GHz})$. The paint thickness measurement was repeated at fifteen random loctions on the rod and the peak detecting algorithm employed to calculate the thickness location, with the results seen in Fig. 4.14. The paint thickness was found to vary between $80 \mu \mathrm{m}$ and $130 \mu \mathrm{m}$ with a mean thickness of $101.94 \mu \mathrm{m}$, in excellent agreement with the manufacturer's specification of between $80 \mu \mathrm{m}$ and $100 \mu \mathrm{m}$ [5]. 


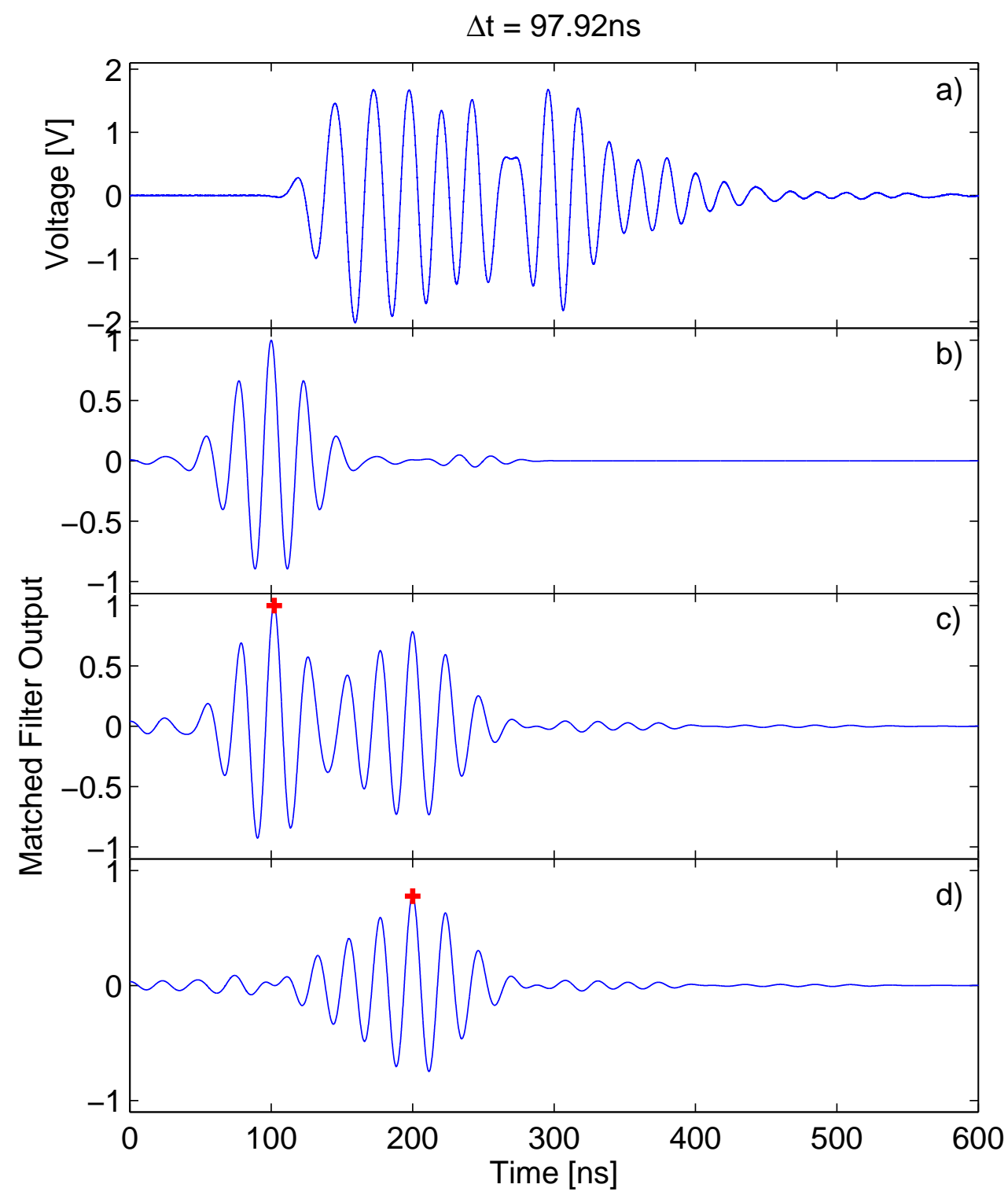

Figure 4.13: a) Oscilloscope trace for pulse-echo measurement on painted rod, b) ideal matched filter output, c) peak for paint surface pulse and d) peak for core pulse with $\Delta t=97.92 \mathrm{~ns}$ corresponding to a paint thickness of $97.92 \mu \mathrm{m}$. 


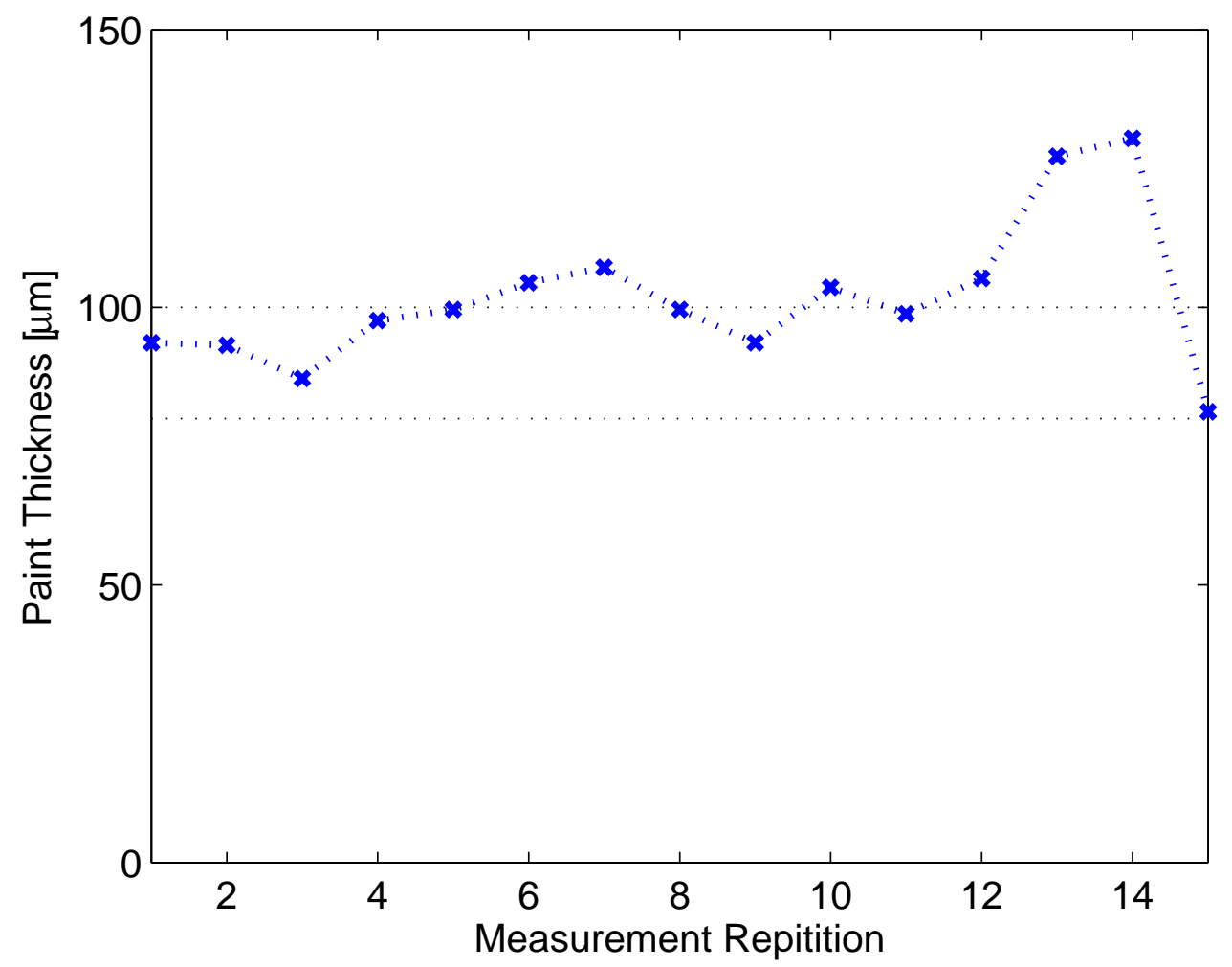

Figure 4.14: Measurements of paint thickness in 15 random positions, black dotted lines represent the expected thickness [5].

\subsection{Conclusion}

This work on coating thickness measurements proved successful in that the thickness of either a sheathed or paint layer could be easily measured accurately with a high frequency ultrasonic immersion transducer. The FEA modelling demonstrated the effectiveness of a pulse echo measurement at measuring layer thickness ultilising the time of flight data and a known velocity. Chirped waveforms and matched filtering were shown to improve the resolution limit of the system. The practicalities of fitting such a system to a production line were beyond the scope of this work but one could imagine that attaching a water bath with o-rings built into 
either side to allow the rod to pass through could be feasible. The use of water jets could provide a means of coupling the transducer to the sample without requiring a bath. 


\section{Chapter 5}

\section{Surface Waves and Crack Detection}

This chapter details the investigation into the use of leaky Rayleigh waves as a probe for detecting surface cracks in materials, particularly glass and fibre reinforced composites (FRC). Finite Element Analysis provided an insight into the propagation of leaky Rayleigh waves and complimentary experiments were performed on a glass sample to develop a reliable technique for launching and detecting Rayleigh waves. An artificial crack was introduced to the sample to verify the techniques. Finally an FRC sample was investigated.

The initial motivation for this portion of work was to develop a technique capable of detecting longitudinal surface cracks in FRC rods, like that shown in Fig. 5.1. In order to reflect a Rayleigh wave off a longitudinal crack the propagation direction must be perpendicular to the crack. In this case the wave should propagate around the circumference of the rod. This requires the Rayleigh wave to successfully propagate around the curved surface which complicates the measurement at high frequencies as it is necessary to satisfy the wavelength/radius ratio of 1.7 [14]. In FRCs such a measurement would be further complicated by the presence of the 


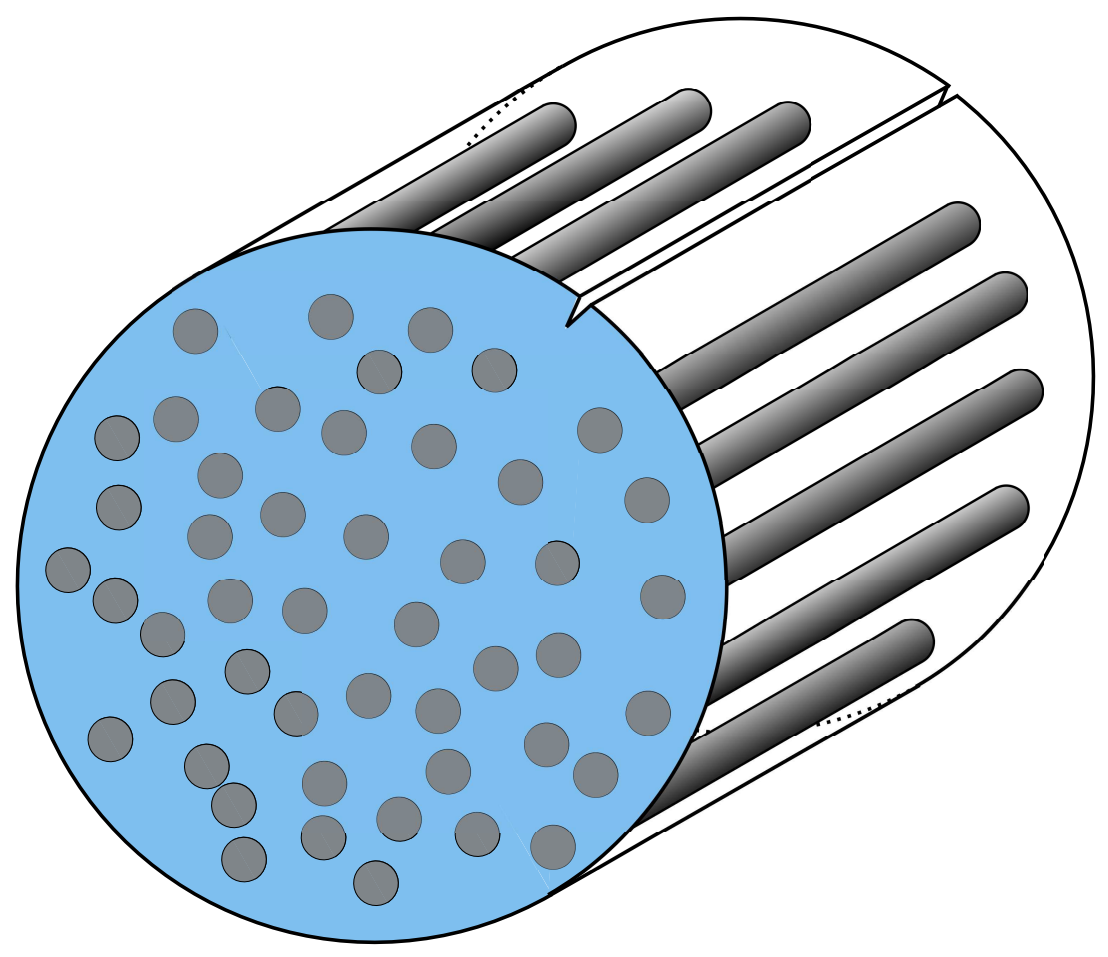

Figure 5.1: Schematic representation of a fibre reinforced composite rod with a longitudinal surface crack. The plane of isotropy is the blue cross section.

glass fibres in the anisotropic rod which would act as multiple scatterers to Rayleigh waves. As a means to gain experience with launching and detecting Rayleigh waves a glass block was investigated as it was smooth, flat and isotropic. 


\subsection{Glass}

\subsubsection{Experimental Velocity Measurements}

A sample of soda lime glass measuring $56 \mathrm{~mm}$ long, $18 \mathrm{~mm}$ wide and $5 \mathrm{~mm}$ deep with parallel faces was used. A pitch-catch measurement was performed across the thinnest dimension. Using the experimental setup from Fig. 4.7(a) a second transducer was dipped into the water from above as a receiver. The velocity of a $\mathrm{P}$ wave in the sample was calculated as $5800 \mathrm{~ms}^{-1}$. To measure the velocity of a shear wave the was inclined at $20^{\circ}$ (past the longitudinal critical angle of $14.8^{\circ}$ ) and the pitch-catch measurement repeated. Refraction caused the propagation direction to change which was accounted for by moving the catching transducer laterally and numerically solving for a range of velocities. The shear velocity was calculated as $3380 \mathrm{~ms}^{-1}$. Having knowledge of the longitudinal and shear velocities the Rayleigh velocity was calculated via (2.1) as $3103 \mathrm{~ms}^{-1}$ with a corresponding critical angle of $28.4^{\circ}$. The $1 / e$ distance of the leaky Rayleigh wave was calculated as $1.3 \mathrm{~mm}$ for $15 \mathrm{MHz}$ and $3.9 \mathrm{~mm}$ for $5 \mathrm{MHz}$.

\subsubsection{Finite Element Analysis}

With the knowledge of the velocities of $\mathrm{P}, \mathrm{S}$ and $\mathrm{R}$ waves an FEA model was created to investigate the generation of Rayleigh waves using critical angles and the propagation of a Rayleigh wave along the surface of the glass plate. The model is seen in Fig. 5.2 and consisted of two identical regions of water and one region of glass, with properties listed in Table 5.1. The two regions of water were required to form an angled boundary to generate the pulse.

At the $28^{\circ}$ water-water boundary a two cycle sinusoidal pulse was generated. This pulse was centred at $5 \mathrm{MHz}$ to simulate the lowest frequency 

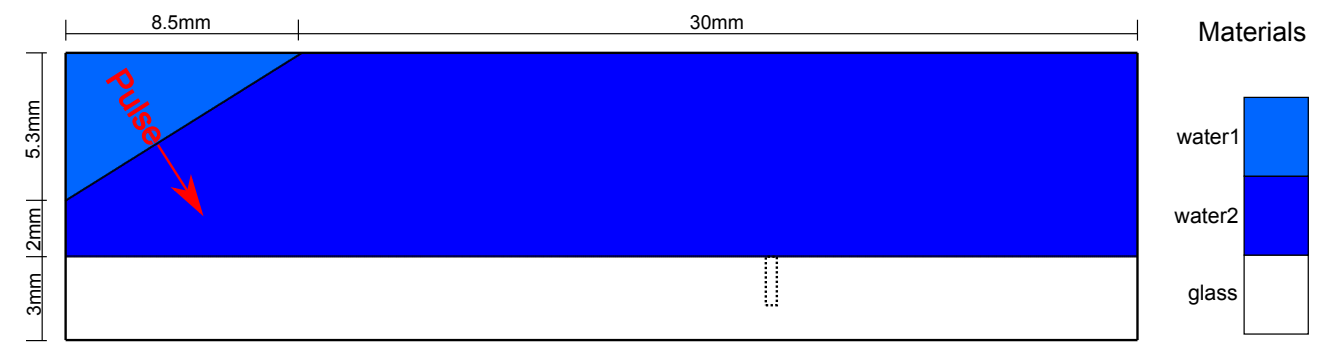

Figure 5.2: PZFlex model for a glass half space immersed in water. A pulse centered at $5 \mathrm{MHz}$ was generated in the water at the Rayleigh angle. The dotted line signifies the location of a crack, utilised in later work.

\begin{tabular}{ccccc}
\hline & & \multicolumn{3}{c}{ Velocity $\left[\mathrm{ms}^{-1}\right]$} \\
\cline { 3 - 5 } Material & Density $\left[\mathrm{kgm}^{-3}\right]$ & Longitudinal & Shear & Rayleigh \\
\hline Water & 1000 & 1480 & - & - \\
Glass & 3000 & 5800 & 3380 & 3103 \\
\hline
\end{tabular}

Table 5.1: Properties of Water and Glass

that could be achieved with the available equipment. In addition, the use of this lower frequency led to an increase in element size and a decrease in the CPU time required to complete the FEA. The width of the water-water boundary was $900 \mu \mathrm{m}$, the same as the $-6 \mathrm{~dB}$ beamwidth for the $20 \mathrm{MHz}$ transducer at that frequency. Four snapshots from the FEA are presented in Fig. 5.3.

The first snapshot $(t=1.5 \mu \mathrm{s})$ shows the pressure pulse travelling through the water at an angle of $28^{\circ}$ relative to the surface normal, the Rayleigh angle for this combination of materials. The wave is mostly planar except for edge effects. These edge effects occur because the elements at the edges act as point sources generating circular, radially expanding waves which can be ignored for the purposes of this simulation. The second snapshot $(t=3 \mu \mathrm{s})$ shows part of the pulse reflecting off the surface of the glass with a phase of $\pi$ relative to the incident wave. There is a shear wave in the 
glass, identified by the curved wavefront, due to the edge effects from the source hence it can be ignored. There is a wave just below the surface of the glass which is suspected to be a Rayleigh wave. The third snapshot $(t=5.7 \mu \mathrm{s})$ shows the full width of the initial pulse having reflected and the suspected leaky Rayleigh wave propagating along the surface with the corresponding leaked energy. Finally the fourth snapshot $(t=9 \mu \mathrm{s})$, shows the suspected leaky Rayleigh wave having propagated along the surface with a significant reduction in magnitude. To confirm that the leaked energy was from a Rayleigh wave it needed to display three characteristics; the surface wave velocity needed to be accurate, the amplitude of the leaked wave needed to decay exponentially and the phase difference between the specular and non-specular reflections needed to be $\pi$ (already observed in the second snapshot). To verify the velocity and exponential decay the pressure was recorded at individual elements $1 \mathrm{~mm}$ above the surface spaced at regular intervals across the width of the simulation., The results are seen in Fig. 5.4.

The peak of each trace was extracted (time and amplitude) and the relationship between the distance and peak time (after the non-specular peak) was confirmed as linear, with a slope calculated as $3115 \mathrm{~ms}^{-1}$. This velocity had an $0.4 \%$ error relative to that calculated earlier of $3103 \mathrm{~ms}^{-1}$, validating the measurement. The relationship between the peak amplitude and distance was investigated and an exponential decay found to be an excellent fit to the data. The $1 / e$ characteristic length of the exponential decay fit was $3.5 \mathrm{~mm}$, in agreement with the expected length of $3.9 \mathrm{~mm}$ for $5 \mathrm{MHz}$.

To experimentally verify the existence of a Rayleigh wave on the glass sample a critical angle reflection measurement was performed. This measurement was pitch-catch in that one transducer was a transmitter and another was a receiver however it differed from those discussed in Section 3.2.2 because it was a reflection measurement rather than transmis- 
sion. Two $20 \mathrm{MHz}$ transducers were positioned at $\theta_{i}=28.4^{\circ}$ (relative to the surface normal), seen in Fig. 5.6(a). To achieve this configuration experimentally a transducer was bolted into a block that was bolted to a single direction rotational stage. This rotational stage was mounted vertically to an XYZ stage allowing control of the location of the transducer in X, Y and $Z$, along with the rotation of the transducer. The rotation of the transducer was equivalent to pitch rather than roll or yaw, seen in Fig. 5.5.

A pulse with centre frequency $f_{c}=15 \mathrm{MHz}$ was transmitted and the catching transducer aligned with the specular reflection. The catching transducer was moved in $100 \mu \mathrm{m}$ steps away from the pitch transducer, parallel to the surface. At each step a voltage-time trace was recorded on the oscilloscope using signal averaging to increase the SNR. As with the FEA the Rayleigh wave velocity was determined by calculating the slope of the linear fit between the distance and peak time for the data on the exponential tail. The velocity was calculated as $3140 \mathrm{~ms}^{-1}$, with a $1.2 \%$ error relative to the velocity of $3103 \mathrm{~ms}^{-1}$ calculated earlier, validating the measurement.

The distance-amplitude data were plotted and two maxima were observed, the first from the specular reflection and second from the leaky Rayleigh wave. The two peaks were separated by $1 \mathrm{~mm}$. The tail was fitted with an exponential decay with a $1 / e$ characteristic distance of $1 \mathrm{~mm}$, close to the expected value of $1.3 \mathrm{~mm}$ for glass at $15 \mathrm{MHz}$. This small change in the characteristic distance can be explained by absorption of the Rayleigh wave in the glass. It is unlikely that the reduction is due to water absorption as the distance travelled in the water by the leaked wave from the surface to the receiving transducer remained constant. To measure the phase of the specular and non-specular reflections a chirp waveform was used with matched filtering and the phases of the pulses were measured as $\pi$ relative to each other. The velocity, exponential decay and phase flip observed in the experimental data confirmed the existence of a leaky 
Rayleigh wave. This work confirmed that a leaky Rayleigh wave could be successfully launched onto an immersed glass sample and detected via critical angle excitation with the equipment available. 

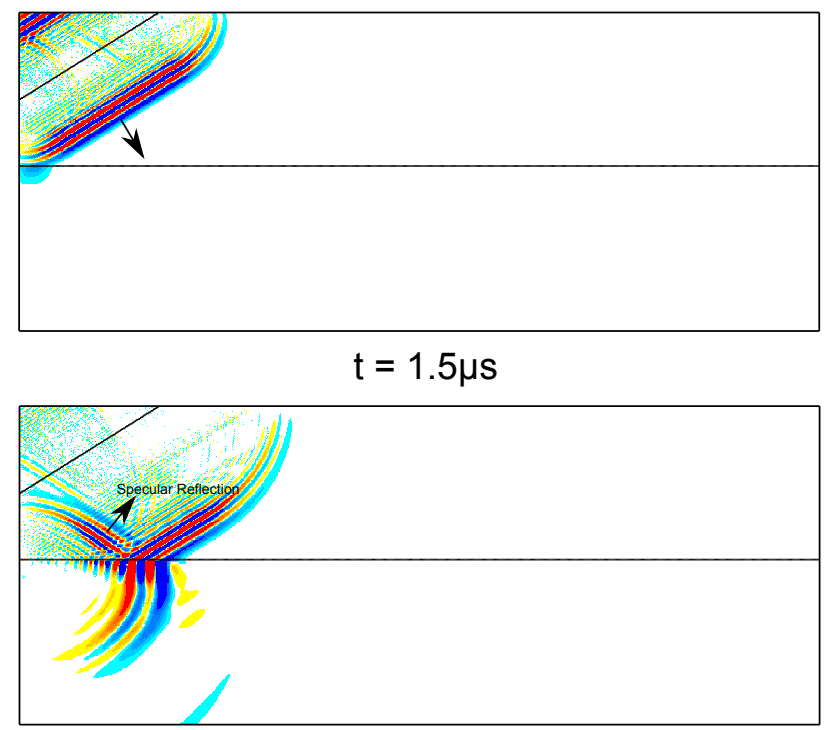

yy stress
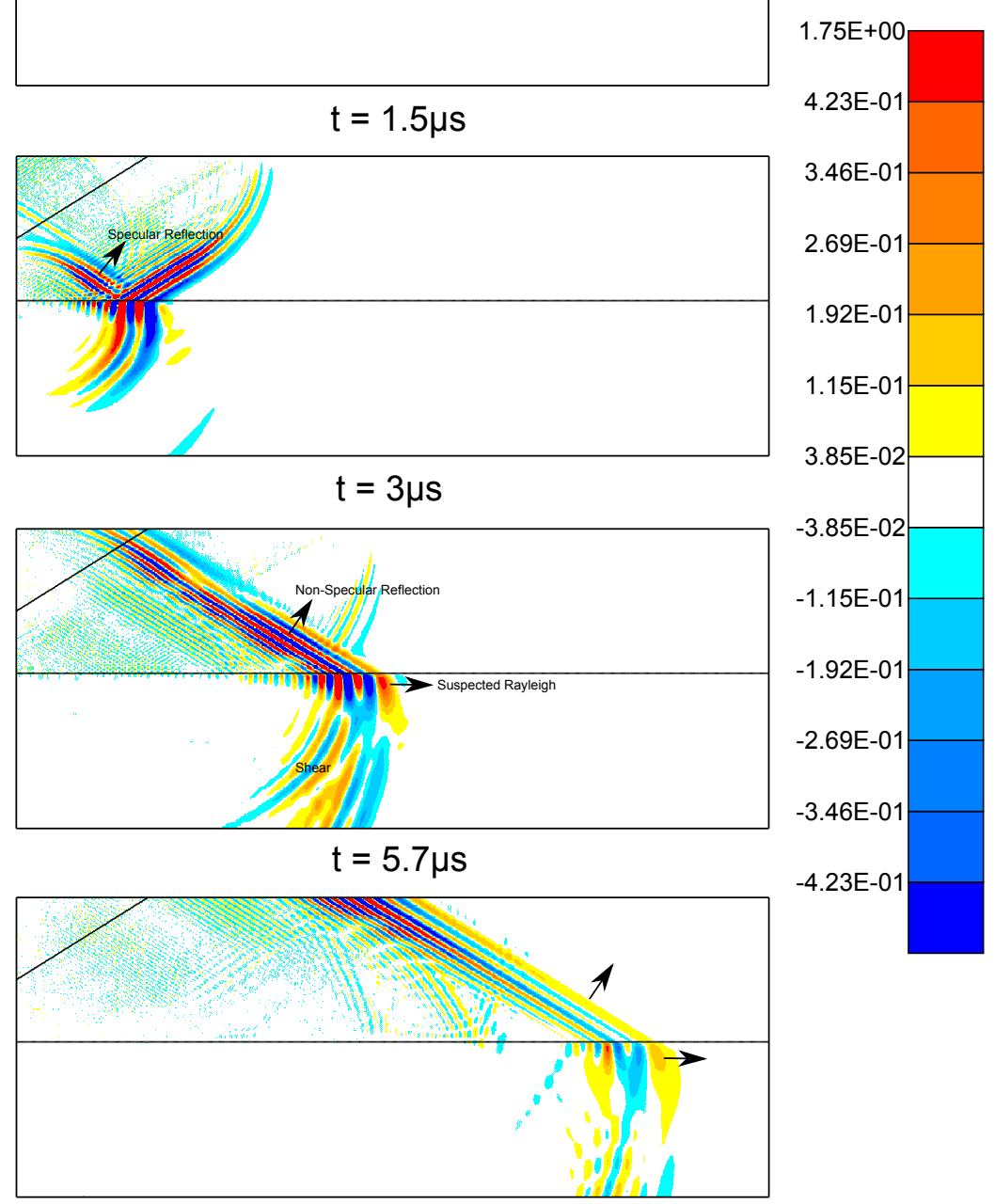

$t=9 \mu s$

Figure 5.3: Snapshots from FEA simulation of glass surface immersed in water. The first snapshot $(t=1.5 \mu \mathrm{s})$ shows the pulse travelling through the water at the Rayleigh angle before partially reflecting in the second snapshot $(t=3 \mu \mathrm{s})$. In the third snapshot $(t=5.7 \mu \mathrm{s})$ a leaky Rayleigh wave is propagating along the surface of the glass and the leaked energy is observed. The amplitude of the leaky Rayleigh wave and the leaked energy have decayed with distance, seen in the fourth snapshot $(t=9 \mu \mathrm{s})$. 

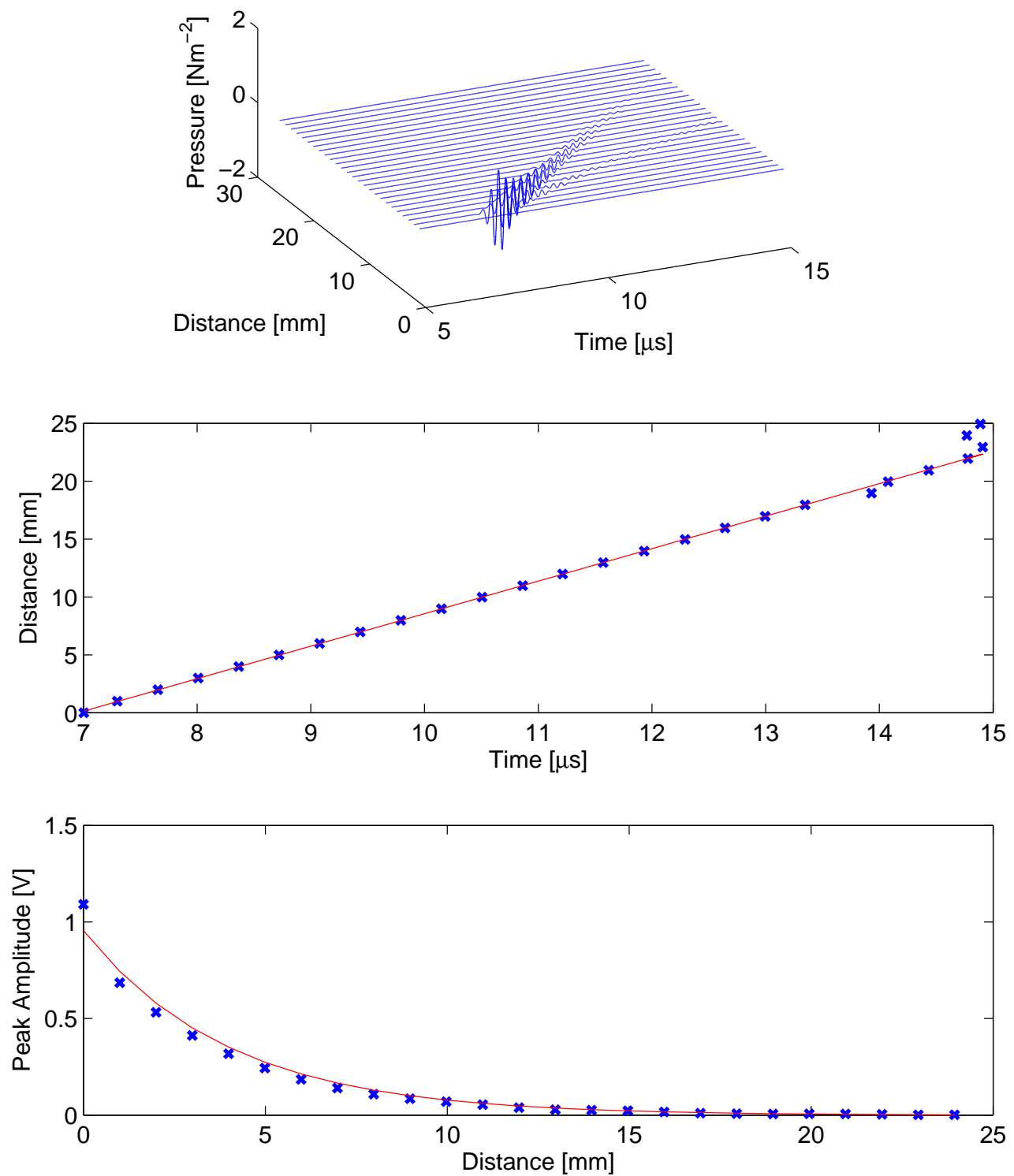

Figure 5.4: Time-Pressure data recorded at regular distance intervals above the surface of the glass in Fig. 5.2. The time of the peak for each distance interval was extracted and a linear fit applied to determine the velocity of $3115 \mathrm{~ms}^{-1}$. The peak amplitude for each distance interval was extracted and an exponential decay fitted, confirming the decaying nature of the leaky Rayleigh wave. 


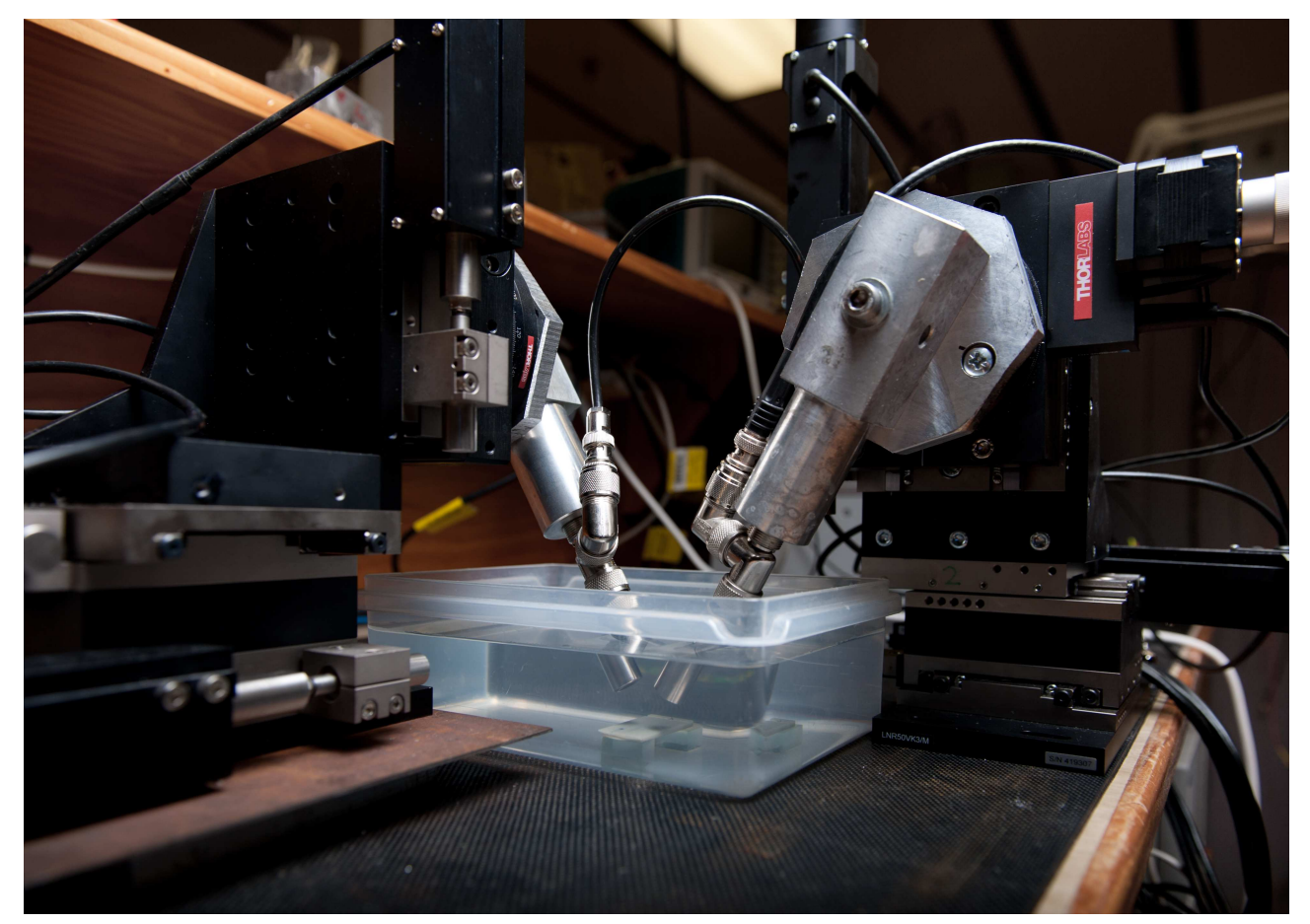

Figure 5.5: Experimental configuration for critical angle excitation of leaky Rayleigh waves on a sample. Each transducer is bolted to a block attached to a rotational stage mounted on an XYZ stage allowing 4 degrees of freedom for the motion of the transducer. The rotational stage was mounted vertically to change the angle of incidence. 


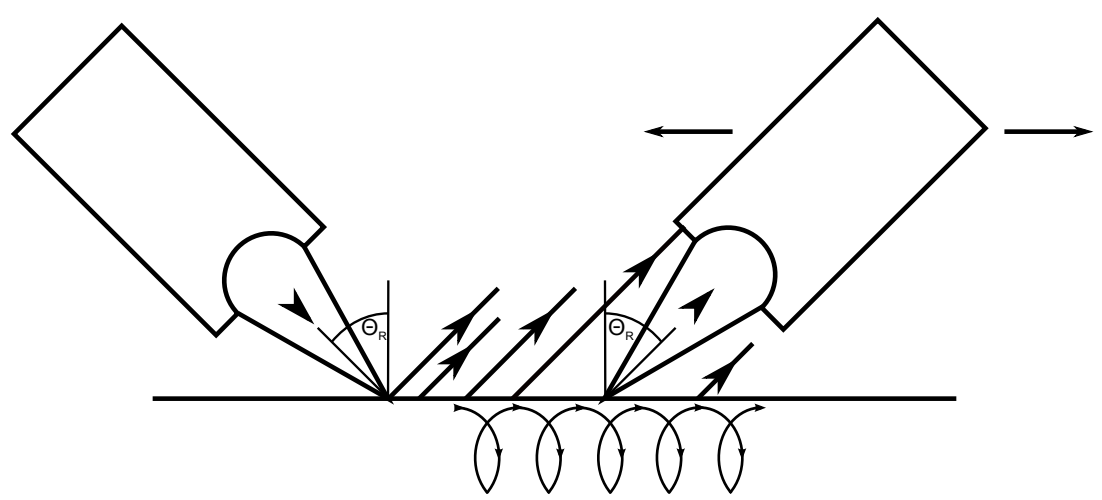

(a) Schematic of pitch-catch measurement to investigate leaky Rayleigh waves. A pitching transducer at the Rayleigh critical angle excites a longitudinal wave in water which is partially specularly reflected. The remaining energy generates a leaky Rayleigh wave and non-specular reflection. A catching transducer is scanned along the surface and measures the specular and non-specular reflections.
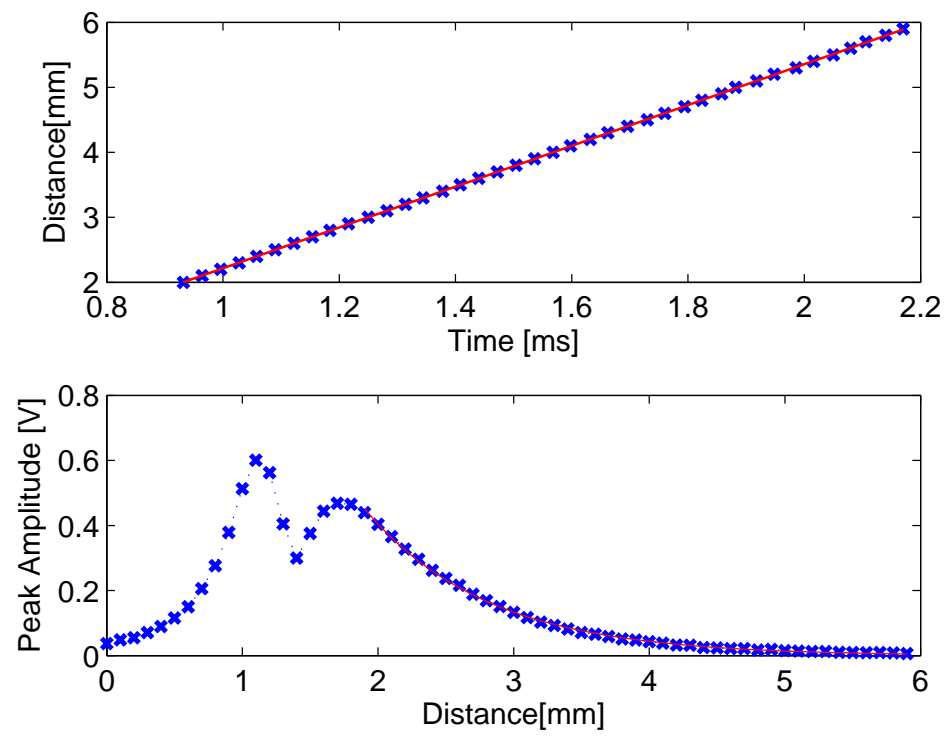

(b) Distance-amplitude data for experimentally observed leaky Rayleigh wave on the surface of glass immersed in water with fitted exponential decay. The distance-time data of the leaky Rayleigh and corresponding linear fit to determine the velocity as $3140 \mathrm{~ms}^{-1}$.

Figure 5.6: Experimental method to verify the existence of a leaky Rayleigh wave on a fluid loaded surface. 


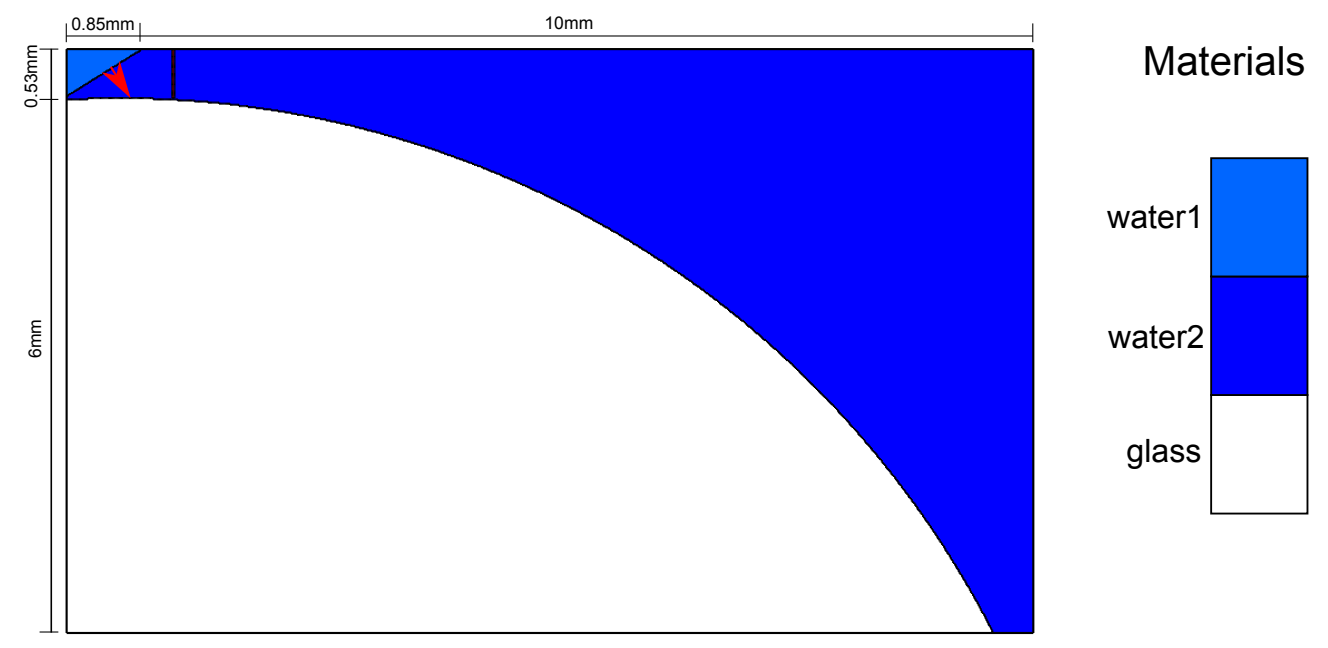

Figure 5.7: Finite Element model to investigate propagation of a leaky Rayleigh wave around a curved surface

\subsection{Circular Cross Section Glass Rod}

Since the FRC rod was circular in cross section an investigation into the propagation of a Rayleigh wave around a curved surface was conducted. An FEA model similar to that in Fig. 5.2 was created but the glass was changed from a flat surface to a circular section, seen in Fig. 5.7. The radius of the circle was $10 \mathrm{~mm}$ and the leaky Rayleigh wave wavelength was $560 \mu \mathrm{m}$, far exceeding the radius/wavelength ratio minimum of 1.7 [14]. Hence the leaky Rayleigh wave was expected to propagate around the rod uninhibited. The centre of the circle was placed such that the ultrasonic pulse would impinge at the Rayleigh angle at the top of the circle. Snapshots from the simulation displaying the acoustic pressure are seen in Fig. 5.8.

The first snapshot shows the pulse having impinged on the glass and due to the edge effects of the source a longitudinal and shear wave have been excited in the glass. A leaky Rayleigh wave is also present at the surface near the top of the circle. In the second snapshot the leaky Rayleigh wave 
is observed to have travelled around the curve with a slight reduction in amplitude due to the energy leakage. Finally in the third snapshot the leaky Rayleigh wave has travelled further and leaked more energy. Of note is the curved nature of the leaked energy.

The pressure was recorded at regular intervals above the surface of the glass and the time of the peak in the leaked energy recorded. From this data a velocity measurement was made, seen in Fig. 5.9. The calculated velocity was $3116 \mathrm{~ms}^{-1}$, in excellent agreement with the velocity of $3115 \mathrm{~ms}^{-1}$ measured for the flat sample FEA. The amplitude of the leaked energy as it travelled was exponentially decaying. This suggested that no other energy losses were occuring and the Rayleigh wave was propagating around the curve with complete transmission.

Attempts were made to experimentally measure the propagation of the leaky Rayleigh wave around a curved rod. However these attempts were unsuccessful. The difficulty in stepping a receiving transducer around the circumference of the rod while ensuring the water path of the leaked energy would remain constant was significant. The physical size of the water bath and the requirement to prevent the electrical connections submerging restricted the range of motion of the transducer, further inhibiting the practicality of the measurement. Such a measurement could be made easier through the use of needle hydrophones as they are omnidirectional, can be placed much closer to the surface and the point at which they are recording is optically visible. 

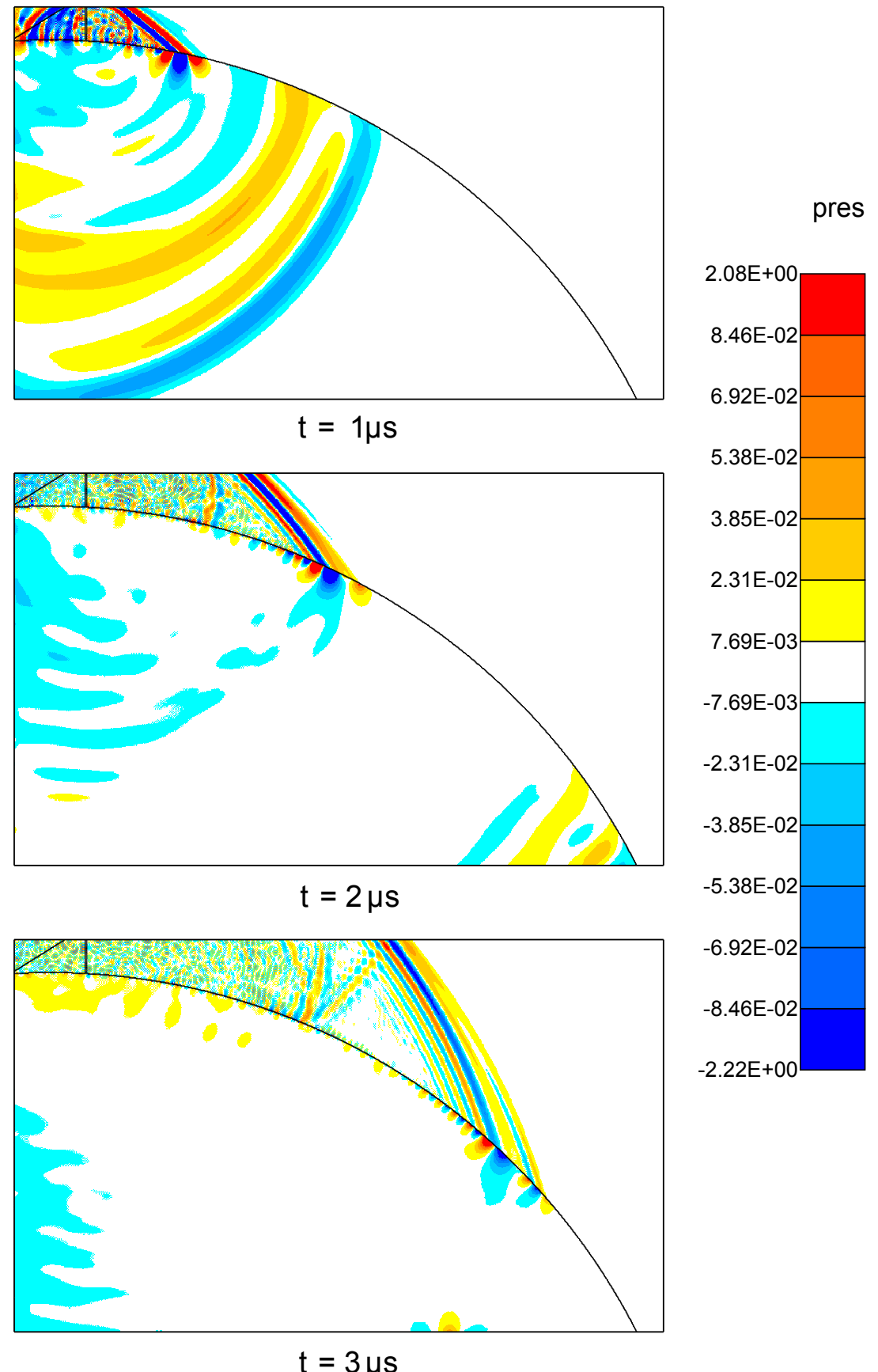

Figure 5.8: Snapshots of a leaky Rayleigh wave propagating around the curved surface of a glass rod, having been excited by a critical angle longitudinal wave. 


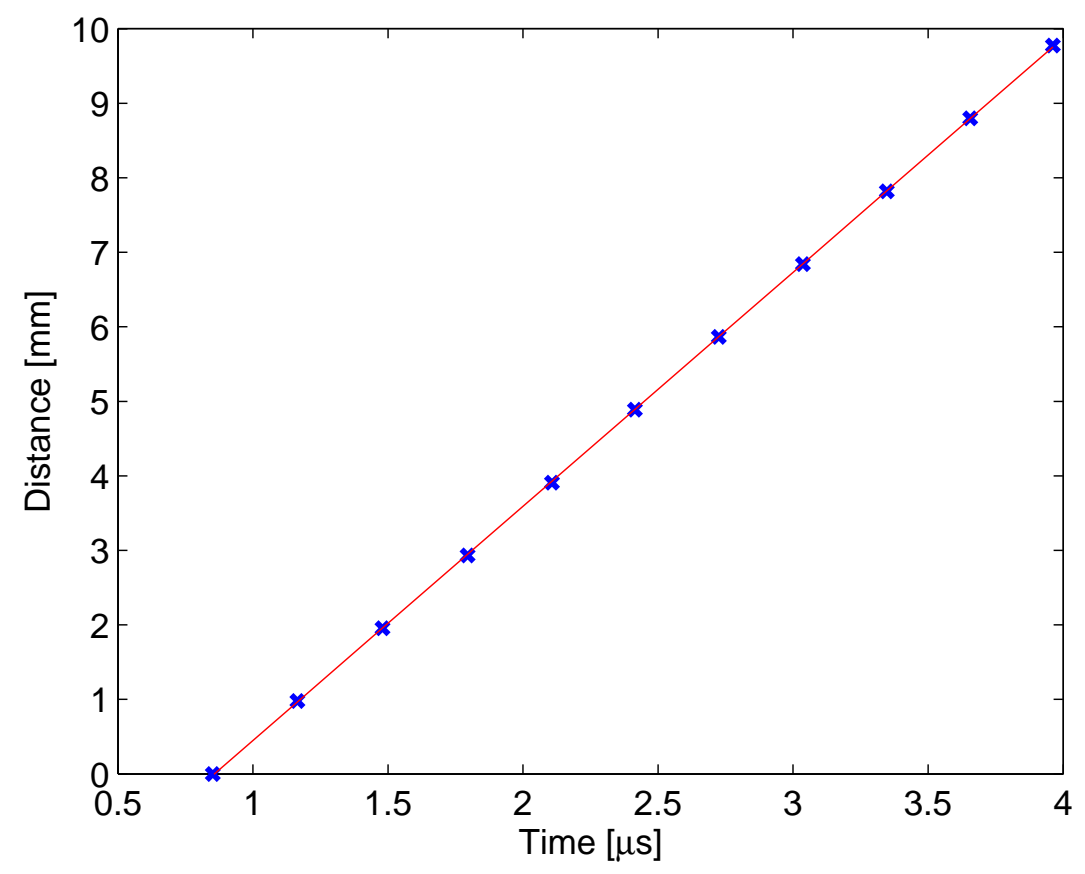

Figure 5.9: Calculated velocity of $3142 \mathrm{~ms}^{-1}$ for leaky Rayleigh wave propagating around a curved glass surface, with an error of $0.9 \%$ relative to the velocity of $3115 \mathrm{~ms}^{-1}$ measured for the flat surface. 


\subsection{Crack Detection}

Rayleigh waves have been used as probes for detecting surface cracks for over half a century [14] and recent studies have investigated the behaviour of Rayleigh waves at cracks [7,42]. The behaviour of a Rayleigh wave incident on a crack is similar to that of a BAW incident on a boundary between media with different acoustic impedances. The Rayleigh wave will also partially reflect and transmit because of the acoustic impedance difference between the surface and the crack (possibly filled with air). However for Rayleigh waves the proportion of energy reflected/transmitted depends on additional factors, namely the depth of the crack relative to a wavelength, the angle of the crack relative to the surface, the width of the crack and the sharpness of the corner where the crack meets the surface. The reflection and transmission phenomena of a leaky Rayleigh wave at a crack were investigated by adjusting the model in Fig. 5.2 to include a crack, realised by including a region of water inside the dotted line. This crack was much deeper than the wavelength. Two snapshots from the FEA are seen in Fig. 5.10.

The first snapshot shows the leaky Rayleigh wave propagating from left to right towards the crack. Again a shear wave is present due to the finite source and edge diffraction. The second snapshot shows the Rayleigh wave having partially reflected off the crack and travelled back towards the left, however the amplitude has reduced since only part of the energy was reflected. Correspondingly there is leaked energy propagating from right to left at the Rayleigh angle. At the crack some energy was mode converted into bulk (longitudinal and shear) waves that propagated into the solid. Some energy diffracted off the tip of the crack into the water as radiating longitudinal waves. The remaining energy was transmitted around the corner of the crack edge and down the crack. The sharpness of the corner defines how much energy will transmit around and down the 
crack, since the corner can be considered to have a radius [14]. Sharp corners will have a very small radius and little energy will propagate around. In contrast, blunt or rounded corners will have a larger radius allowing more energy to propagate around the corner. Since the crack here was much deeper than wavelength the reflected energy would be frequency independent. To contrast, if the crack was shorter than a wavelength, long wavelength Rayleigh waves would pass undernearth the crack while short wavelengths would reflect. This is the motivation for using spectroscopy $[6,7,42]$ in crack detecting measurements as the depth of the crack could be determined by measuring the frequency content of reflected and transmitted Rayleigh waves.

From this simulation it was concluded that there are two potential methods for detecting a crack much deeper than a wavelength. The first method is a pulse-echo measurement using a single transducer to excite a Rayleigh wave then detect any energy that reflected off the crack and leaked back to the transducer. The transducer would already be at the critical angle so the leaked energy from the reflection would be detected. The timing of the reflected pulse would assist in determining the location of the crack. The second method is a pitch-catch measurement that would involve offsetting the catching transducer, measuring the leaked energy signal and comparing this to a crack free surface. If the signal amplitude deviated significantly from the exponential decay a crack would be indicated.

To investigate the efficacy of these methods an artificial crack was created in the glass sample by cutting a notch into the surface of the glass plate with a dicing saw. The crack measured $1 \mathrm{~mm}$ deep and $200 \mu \mathrm{m}$ wide hence it was estimated that Rayleigh waves with frequencies above $3 \mathrm{MHz}$ would be fully reflected.

To test the pulse-echo method a $20 \mathrm{MHz}$ transducer was set to the criti- 
cal angle and aimed at the surface of the glass approximately $1 \mathrm{~mm}$ away from the crack. A $15 \mathrm{MHz}$ signal was launched and observed on the oscilloscope. To confirm that this signal was from a reflected leaky Rayleigh wave the velocity was measured by stepping the transducer closer to the crack in $100 \mu \mathrm{m}$ intervals and recording the time of the peak for each oscilloscope trace. The relationship between the step distance and peak time would have to be linear with a slope half that of the expected $\sim 3100 \mathrm{~ms}^{-1}$, due to the actual change of distance being twice the step distance. A linear relationship is seen in Fig. 5.11(b) however the measured velocity was $3206 \mathrm{~ms}^{-1}, 106 \mathrm{~ms}^{-1}$ greater than the expected velocity.

The difference was suspected to be from diffraction off the tip of the crack travelling directly through the water to the transducer, seen as the dotted line in Fig. 5.11(a). However this was unlikely as the lens and the angle of the transducer ensured the strongest signals received would be propagating along the beam axis. Through trigonometry it was shown that a wave diffracted off the crack corner that travelled directly to the transducer face would result in a slower velocity measured when the transducer was stepped. Since the measured velocity was greater than that expected the idea of the diffracted wave was subsequently invalid. This pulse-echo method was proven successful and the range over which a crack could be detected was approximately $1 \mathrm{~mm}$ due to the small $1 / e$ distance for glass in water and only partial reflection of the energy at the crack.

The pitch-catch method seen in Fig. 5.6(a) was also evaluated for crack detection. A measurement was first performed on a part of the glass slide with no crack present. The amplitude of the peaks for the leaky Rayleigh wave were extracted and fitted an exponential decay, seen in Fig. 5.12 as the blue line.

The pitching transducer was moved so that its focal spot was approxi- 
mately $3 \mathrm{~mm}$ away from the crack. The pitch-catch measurement was repeated and the peak amplitudes plotted as the green line, which follows the decaying exponential trend until $1.8 \mathrm{~mm}$, where the amplitude rapidly decreases. This signified a reduction in the amplitude of the leaked energy hence the leaky Rayleigh wave had reflected off the crack and not propagated past. The measurement was repeated with the distance between non-specular peak and crack reduced by $500 \mu \mathrm{m}$ each time, seen as the red, cyan, magenta and yellow lines. Each repitition shows a sudden decrease in peak amplitude at $1.3 \mathrm{~mm}, 0.8 \mathrm{~mm}, 0.3 \mathrm{~mm}$ and $0 \mathrm{~mm}$, validating the reflection pitch-catch method of surface crack detection for a range of up to approximately $2 \mathrm{~mm}$. 


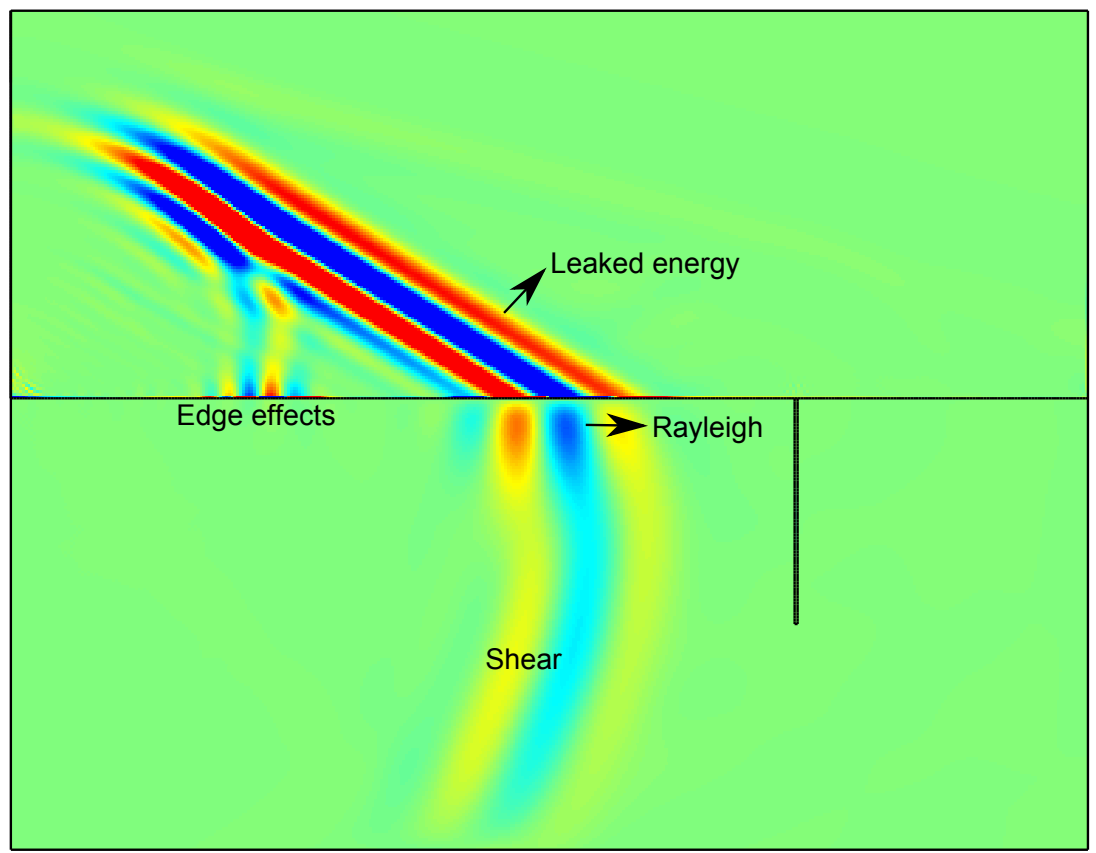

(a) Leaky Rayleigh wave propagating towards a crack.

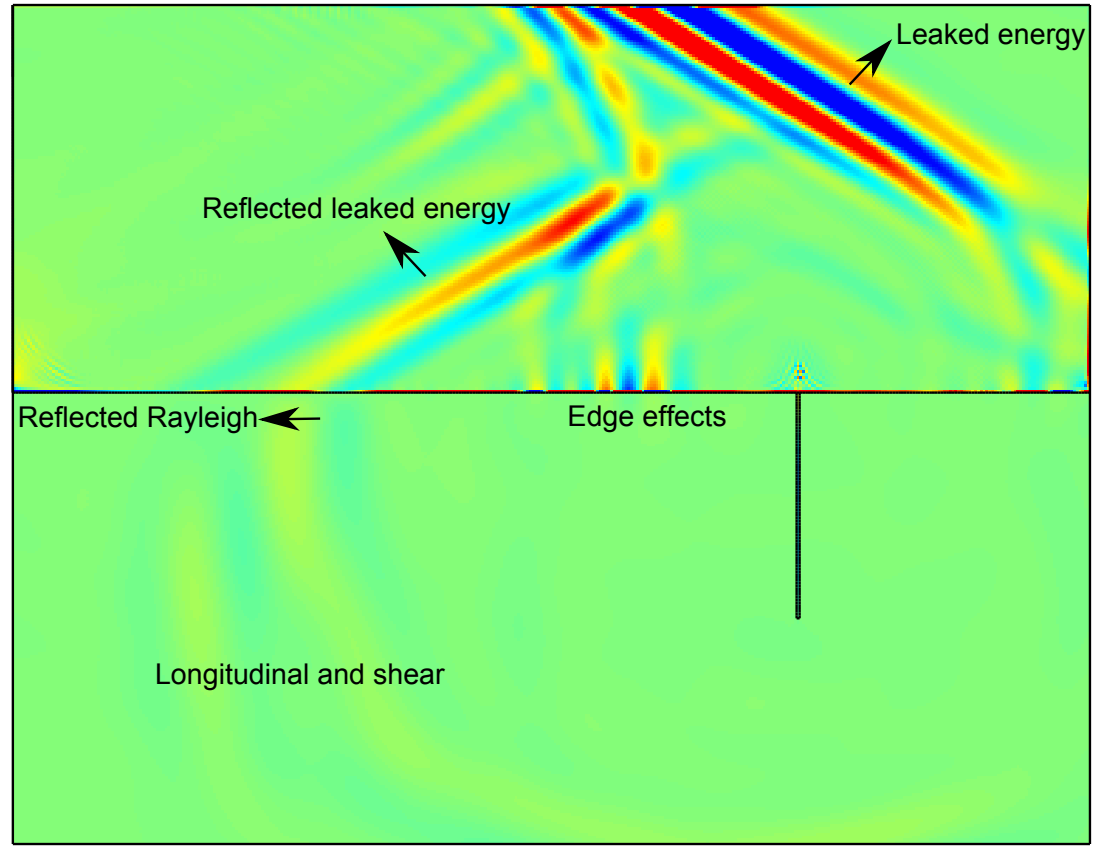

(b) Leaky Rayleigh wave having partially reflected from a crack and mode converted into bulk acoustic waves.

Figure 5.10: Simulation of leaky Rayleigh wave behaviour at a surface crack in glass. 


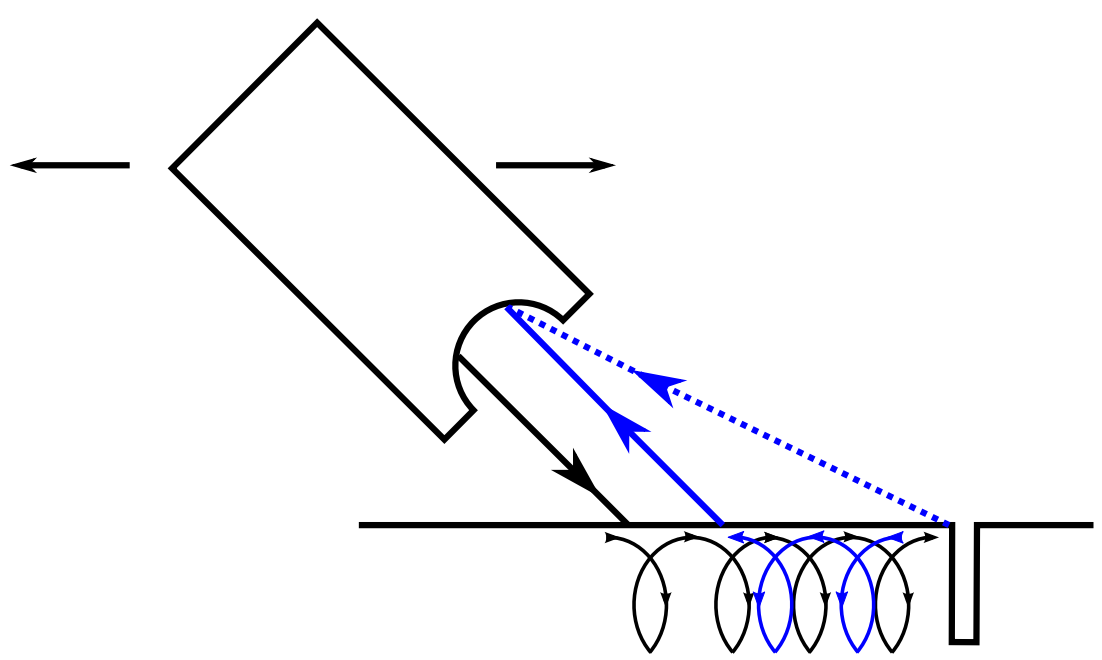

(a) Schematic of pulse-echo crack detection measurement where a leaky Rayleigh wave is excited at the critical angle (black) then is reflected off of a crack and leaks back to the transducer (blue). The dotted line is the propagation path of the tip diffraction from crack to transducer.

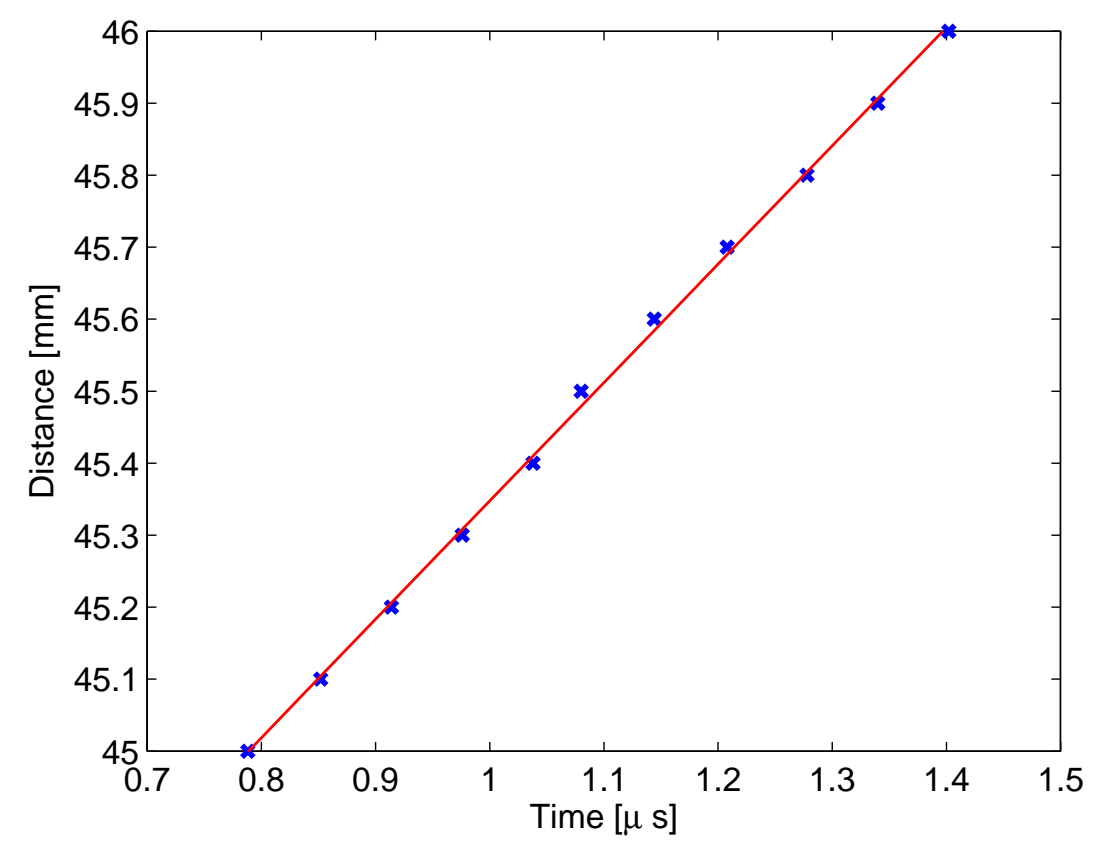

(b) Measurement of Rayleigh velocity where the linear fit has a slope $1643 \mathrm{~ms}^{-1}$ but the propagation distance was twice indicated hence the velocity actually is $3286 \mathrm{~ms}^{-1}$.

Figure 5.11: Pulse-echo Measurement for crack detection on glass. 


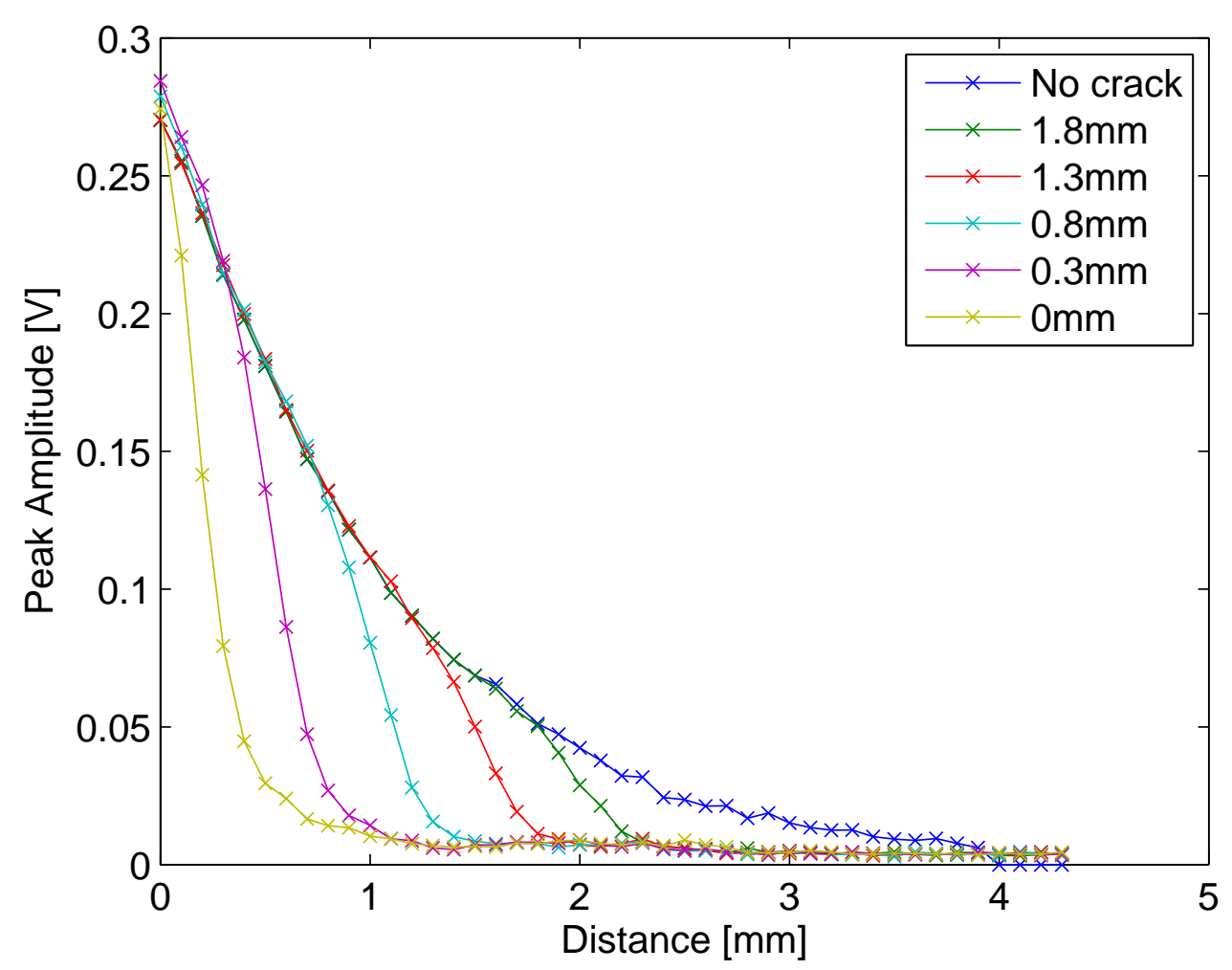

Figure 5.12: Peak amplitude of pulse received in reflection pitch-catch measurement. The blue line is the result for a smooth surface, where the amplitude decays exponentially with distance. The green line is for the measurement where a surface crack was $1.8 \mathrm{~mm}$ from the peak of the nonspecular reflection, identified by the distinct drop in amplitude away from the exponential. The red, cyan, magenta and yellow lines are the same as the green line except the crack was $1.3,0.8,0.3 \& 0 \mathrm{~mm}$ away from the non-specular peak, respectively. 


\subsection{Fibre Reinforced Composite Rods}

\subsubsection{FEA of Wave Propagation}

A sample of a glass FRC was obtained with surface cracks roughly one tenth of a millimetre wide. As the rods consist of long, thin $(20 \mu \mathrm{m})$ glass fibres embedded in an epoxy resin the rods are significantly stronger in their long dimension and are considered anisotropic. The other two dimensions are identical and form a plane of isotropy, seen as the blue cross section in Fig. 5.1. This type of anisotropy is defined transversely isotropic [43] while the surface crack is in the longitudinal direction. To detect a crack with this orientation, the Rayleigh wave would have to travel around the circumference of the rod, in the plane of isotropy. The FEA in this section was then conducted on models based on this cross section. In a 2D respresentation these fibres present multiple circular scatterers to a Rayleigh wave .

To successfully model the FRC rod a knowledge of the fibre density was required. A cross section was cut and polished with a diamond paste to a finish of $1 \mu \mathrm{m}$ using a Struers Tegrapol machine. Micrographs were taken using an Olympus ColorView $\mu$ camera mounted to an Olympus BX51 microscope. The micrographs can be seen in Fig. 5.13(a). Image capture software was employed to determine the radii of the fibres, calculated as $\sim 10 \mu \mathrm{m}$, in agreement with the manufacturer's specification [5]. A circle detection algorithm [44] in Matlab was employed to calculate the fibre density from the micrograph images. The algorithm output the centres and radii of the detected circles, seen in Fig. 5.13(c). The detected circles were overlayed on the original image and the accuracy of the algorithm was very good. The vast majority of circles, with their entire area within the bounds of the image, were detected. The exceptions were those on the edge of the images where less than half of their area was in the image. These exceptions are to be expected as all images must have an edge. Additional circles were detected that didn't correspond to a fibre, however 
they amounted less than $10 \%$ of the circles detected. The density was calculated by summing the number of pixels within the detected circles and dividing by the total number of pixels with a result of $54 \%$. This result was in agreement with manufacturer's quoted range of $50 \%-85 \%$ [4].

To complement the micrographs SEM images were created using a JEOL JSM-5300LV, seen in Fig. 5.14. The circle detection algorithm was applied to a portion of Fig. 5.14(a) and yielded similar results to the micrographs. The density was calculated as $53 \%$ and there were significantly less false positive results. However a greater number of circles missed detection. One advantage of the SEM images was the clear identification of the extent of a surface crack, identified as a lack of fibres extending $2 \mathrm{~mm}$ down from the surface of the rod. This depth would determine the frequency of the Rayleigh wave. A second advantage was the high magnification and resolution achieved for an image of the surface of the rod, seen in Fig. 5.14(b). The $10 \mu \mathrm{m}$ radius fibres are present at the surface of the rod, with epoxy filling the gaps between fibres resulting in a smooth surface. This proximity of fibres to the surface suggests that the surface would present as a series of sub wavelength diffusive scatterers, as opposed to the approximation of a flat surface or specular reflector. 


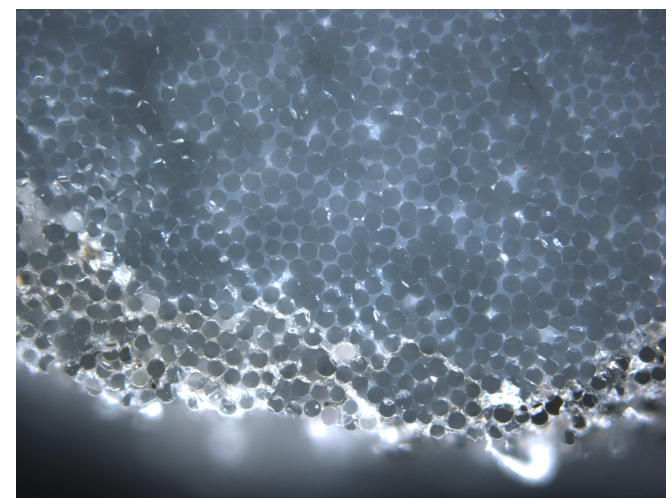

(a) Micrograph of polished cross section of FRC rod taken at surface of rod.

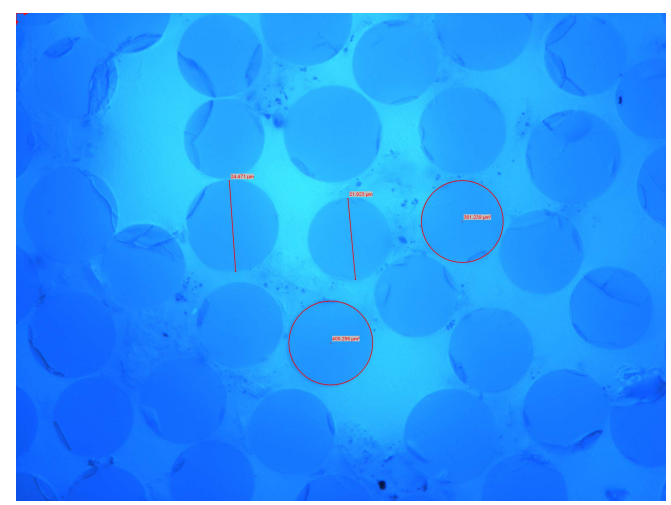

(b) Micrograph of polished cross section of FRC rod, displaying fibre dimensions.

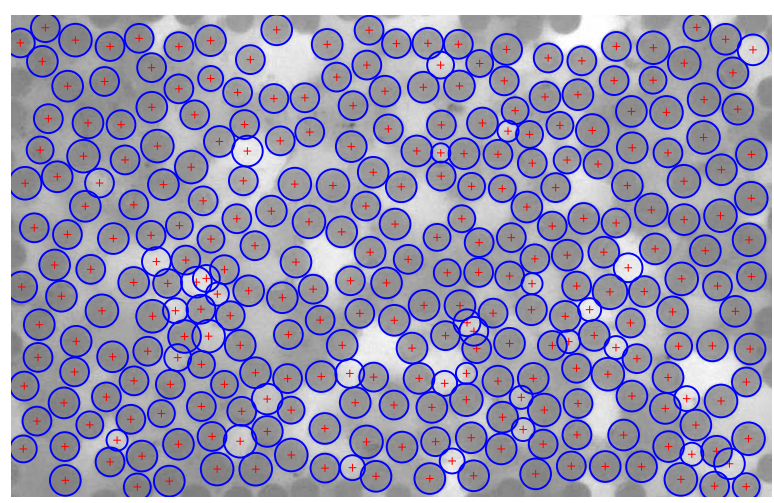

(c) Result of circle detection algorithm applied to micrograph of FRC rod. Resulting fibre density is $54 \%$.

Figure 5.13: Micrographs of a cut and polished cross section of the fibre reinforced composite rod. 


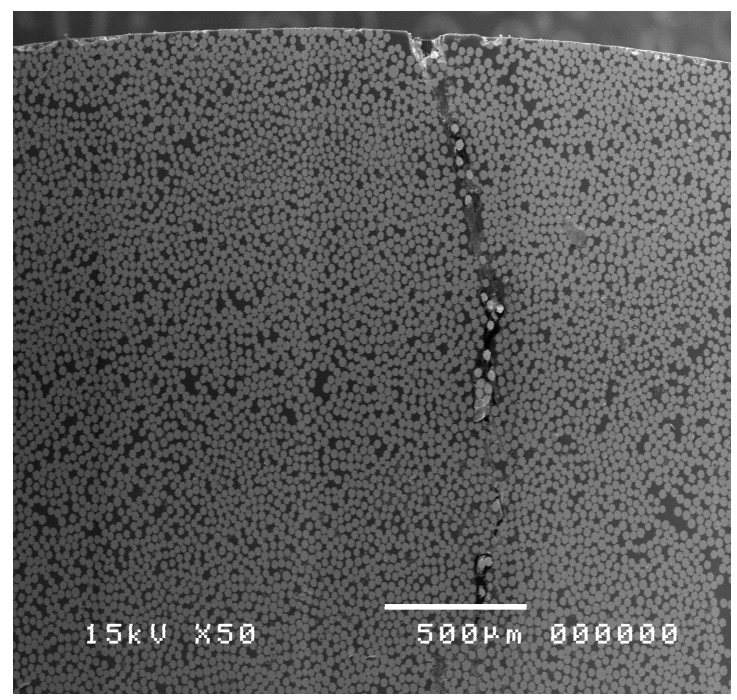

(a) SEM of polished cross section of fibre reinforced composite rod, displaying surface of rod, distribution of fibres and surface crack

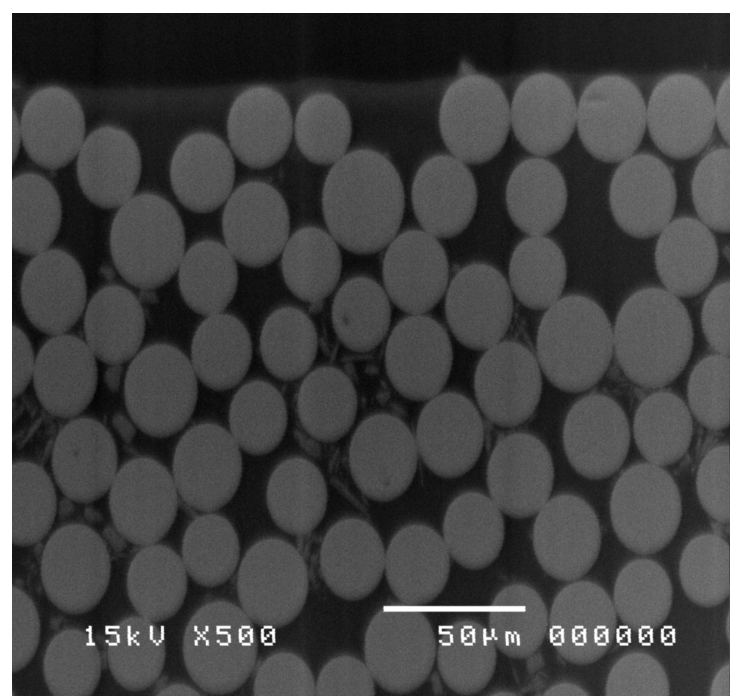

(b) SEM of surface of polished cross section of fibre reinforced composite rod, distribution of fibres and surface crack.

Figure 5.14: SEM images of cut and polished cross section of fibre reinforced composite rod. 


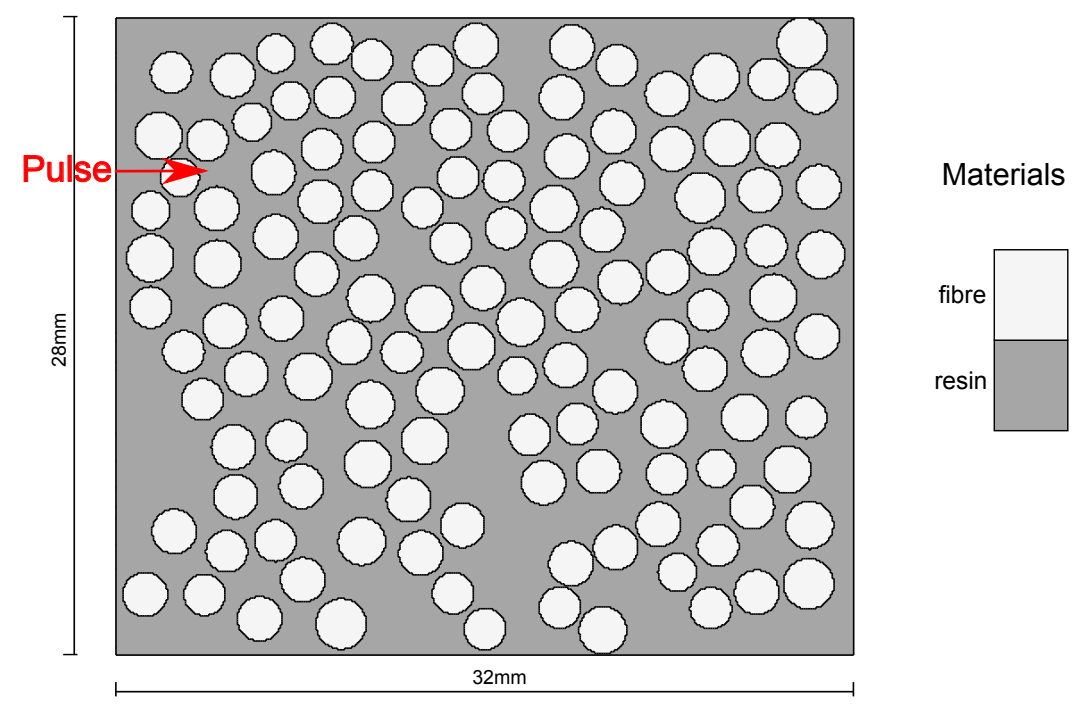

Figure 5.15: FEA model of plane of isotropy in FRC rod - glass fibre circles embedded in resin with distribution taken from circle detection algorithm.

From the circle detection algorithm an FEA model of the cross section of the FRC rod was generated, seen in Fig. 5.15. A single cycle sinusoid was generated at the left boundary. The top and bottom boundaries were symmetric to ensure there were no edge effects. To investigate the effects of scattering the frequency was varied starting at $5 \mathrm{MHz}$ and sequentially doubled up to $80 \mathrm{MHz}$. Snapshots from the FEA are presented in Fig. 5.16.

The first snapshot (top left) for $5 \mathrm{MHz}$ shows the pulse propagating across the model from left to right with small pertubations to the wave due to scattering. However the affect is small due to the scatterer dimension being much less than the wavelength. The second snapshot (top right) shows the pulse having propagated past the model, with small amounts of energy reverberating between scatterers. The same behaviour is observed for the $10 \mathrm{MHz}$ snapshots, but the effect of the scatteres has increased in the first snapshot, seen as a reduction in the pressure amplitude. The reverberations in the second snapshot are minimal. At $20 \mathrm{MHz}$ the amplitude 
has reduced further in the first snapshot while the reverberations have increased in amplitude. In the second snapshot the reverberations after the pulse has passed are much larger. This decrease in pulse amplitude and increase in reverberation is again observed at $40 \mathrm{MHz}$, due to the scattering changing from specular to diffusive. At $80 \mathrm{MHz}$ the first snapshot shows the pulse beginning to propagate aross the model and the amplitude has already reduced. The second snapshot shows the pulse two thirds of the way across the model with its amplitude reduced to the level of the reverberations. The result of this FEA modelling was that low frequencies must be used to effectively propagate an ultrasonic wave through an FRC sample.

\subsubsection{Measurement of Acoustic Properties}

Measurements of the acoustic properties of the FRC rod were made using the same techniques used for the glass. The rod was $22 \mathrm{~mm}$ in diameter with a fibre density of $\sim 53 \%$ [4], hence an ultrasonic wave travelling across the rod could be scattered by potentially approximately 600,000 individual fibres resulting in a significant reduction of signal. To ensure a signal of sufficient magnitude would propagate through the sample, a section of rod was milled to form a plate. The fibres were parallel to the longest dimension of $64.55 \mathrm{~m}$. The plate was $22.14 \mathrm{~mm}$ wide and $2.69 \mathrm{~mm}$ thick resulting in a volume of $3.84 \times 10^{-6} \mathrm{~m}^{3}$. The mass of the sample was measured as $7.733 \mathrm{~g}$, corresponding to a density $\rho=2011 \mathrm{kgm}^{-3}$, within the range quoted by the manufacturer of $1900-2100 \mathrm{kgm}^{-3}$ [4]. The longitudinal and shear velocities across the fibres were measured in a pitchcatch experiment, indentical to that used for the glass, from which the Rayleigh velocity was calculated. The theoretical $1 / e$ characteristic distance of a Rayleigh wave was calculated as $2.2 \lambda$, an order of magnitude smaller than glass. This small characteristic length would result in the 


\begin{tabular}{cccccccc}
\hline Density $\left[\mathrm{kgm}^{-3}\right]$ & \multicolumn{2}{c}{$V_{P}\left[\mathrm{~ms}^{-1}\right]$} & \multicolumn{2}{c}{$V_{S}\left[\mathrm{~ms}^{-1}\right]$} & \multicolumn{2}{c}{$V_{R}\left[\mathrm{~ms}^{-1}\right]$} \\
\cline { 2 - 7 } & Across & Along & Across & Along & Across & Along \\
\hline 2011 & 3370 & 5400 & 1770 & - & 1640 & - \\
\hline
\end{tabular}

Table 5.2: Properties of Cracked Fibre Reinforced Composite Rod

Rayleigh wave leaking $95 \%$ of its energy after travelling only $6.6 \lambda$ along the surface. The longitudinal velocity along the fibres was measured to highlight the anisotropy of the sample and the velocities for the sample are presented in Table 5.2. The shear and Rayleigh velocities along the fibres were not measured because they were not required.

To investigate the propagation of a Rayleigh wave along the surface of the FRC sample an FEA model was created identical to that used for glass in Fig. 5.2 except the solid properties matched the experimentally measured macroscopic values for the FRC rod. The width of the source was set to match the beam width of the $10 \mathrm{MHz}$ transducer at $5 \mathrm{MHz}$ to best model the experimental setup. The solid was isotropic as no fibres were included which enabled the element size to be increased and resulted in the simulation processing significantly faster. Snapshots from the simulation are seen in Fig. 5.17.

The first and second snapshots are similar to those of the glass with the pulse travelling through the water and partially reflecting off the surface as a Rayleigh wave is generated. The amplitude of the Rayleigh wave is significantly smaller than the reflected wave. In the third snapshot the Rayleigh wave is faint due to the energy having leaked very quickly, as expected from theory. On the left side of the model at the boundary between the two water regions residual errors of the model begin to accumulate and spread over the model. In the fourth snapshot the leaky Rayleigh wave has travelled along the surface however the Rayleigh wave is not 
visible, only the leaked energy. The intensity scale was changed to better view the leaky Rayleigh wave but this amplified the effect of the residual errors such that the entire simulation was swamped with blue and red such that no distinct waves were visible. Irrespective of the scale the leaky Rayleigh wave did not propagate far before significantly losing energy, as expected. The simulation was repeated with the solid replaced by a distribution of glass circles in epoxy like that in Fig. 5.15 however the residual errors of the model were much greater and the entire model was swamped with red and blue. This was assumed to be an error relating to the large number of elements required to accurately model a circle. To counter this problem the circles were changed to squares yet the swamping still occured.

\subsubsection{Experimental Measurement of Rayleigh Waves}

To experimentally verify the existence of a Rayleigh wave on the surface of the sample (propagating across the fibres) the same pitch-catch technique used for the glass was employed. As a low frequency was desired to boost the $1 / e$ distance and decrease scattering, the $10 \mathrm{MHz}$ transducer was used to pitch and a $20 \mathrm{MHz}$ transducer to catch. Both were set to the Rayleigh critical angle of $65^{\circ}$. A $6 \mathrm{MHz} 5$ cycle sinusoid was used as an excitation pulse to ensure the pulse was of significant energy and to narrow the bandwidth of the pulse. The Hann window provided a distinct peak. It was observed in a pulse-echo measurement that the received signal was from many scatterers, as expected from the SEM images. The catching transducer was aligned with the specular reflection and then stepped away in $100 \mu \mathrm{m}$ steps. The distance-amplitude data are presented as the blue crosses in Fig. 5.18.

There are two peaks present however the second is less pronounced. This 
was expected as the $1 / e$ characteristic distance of the Rayleigh wave was only $2.2 \lambda$ or $\sim 610 \mu \mathrm{m}$ at $6 \mathrm{MHz}$. Since the leaky Rayleigh wave's amplitude decayed quickly the exponential decay of the non-specular reflection could not be separated from the Gaussian like beam shape of the specular reflection. In an attempt to isolate the non-specular reflection a blocker was used, an idea proposed in the literature [14, 22, 45]. A specular reflection blocker was created by sandwiching a section of closed cell foam between two microscope cover slips, seen in Fig. 5.19. The blocker was $1 \mathrm{~mm}$ thick and was approximately $100 \mu \mathrm{m}$ above the plane it rested on. These materials were chosen because they were thin and regular in shape. The glass was incorporated to reflect the majority of energy due to its significant acoustic impedance difference relative to the water. The closed cell foam served to absorb and/or reflect any energy that transmitted through the first cover slip.

The blocker was attached to an 'L' shaped stand and placed on the sample. A pulse-echo measurement was performed to aim the pitching transducer at the base of the blocker. Another pulse-echo measurement aimed the catching transducer at the base of the blocker on the other side, with the relative distance between this location and the specular reflection noted as $0.9 \mathrm{~mm}$. The catching transducer was moved $5 \mathrm{~mm}$ towards the blocker, past the location of the specular reflection, effectively preventing any acoustic energy in the water propagating at the Rayleigh angle to reach the transducer. A pitch-catch measurement was performed where the catching transducer was moved in $100 \mu \mathrm{m}$ steps parallel to the sample surface, along the direction of propagation of the Rayleigh wave. An oscilloscope trace was recorded for each step and the amplitude of the peak of each trace was extracted, seen as red crosses in Fig. 5.18. For the distance equal to 0 , the catching transducer was in the shadow region of the blocker, hence no leaky Rayleigh wave energy was expected to impinge on the transducer. However energy was present, identified by a non-zero am- 
plitude, which was thought to be from diffraction at the corner at the base of the blocker. As the transducer was stepped more diffracted energy impinged on the transducer at angles closer to the beam axis of the transducer, resulting in a larger amplitude pulse. At $4.9 \mathrm{~mm}$ the amplitude peaked, as observed without the blocker, because the catching transducer was 'aiming' at the surface of the FRC at the base of the blocker. As the distance was increased the amplitude fell away, as expected. The velocity was determined by extracting the time of each peak for each trace after $4.9 \mathrm{~mm}$, seen in Fig. 5.20. 


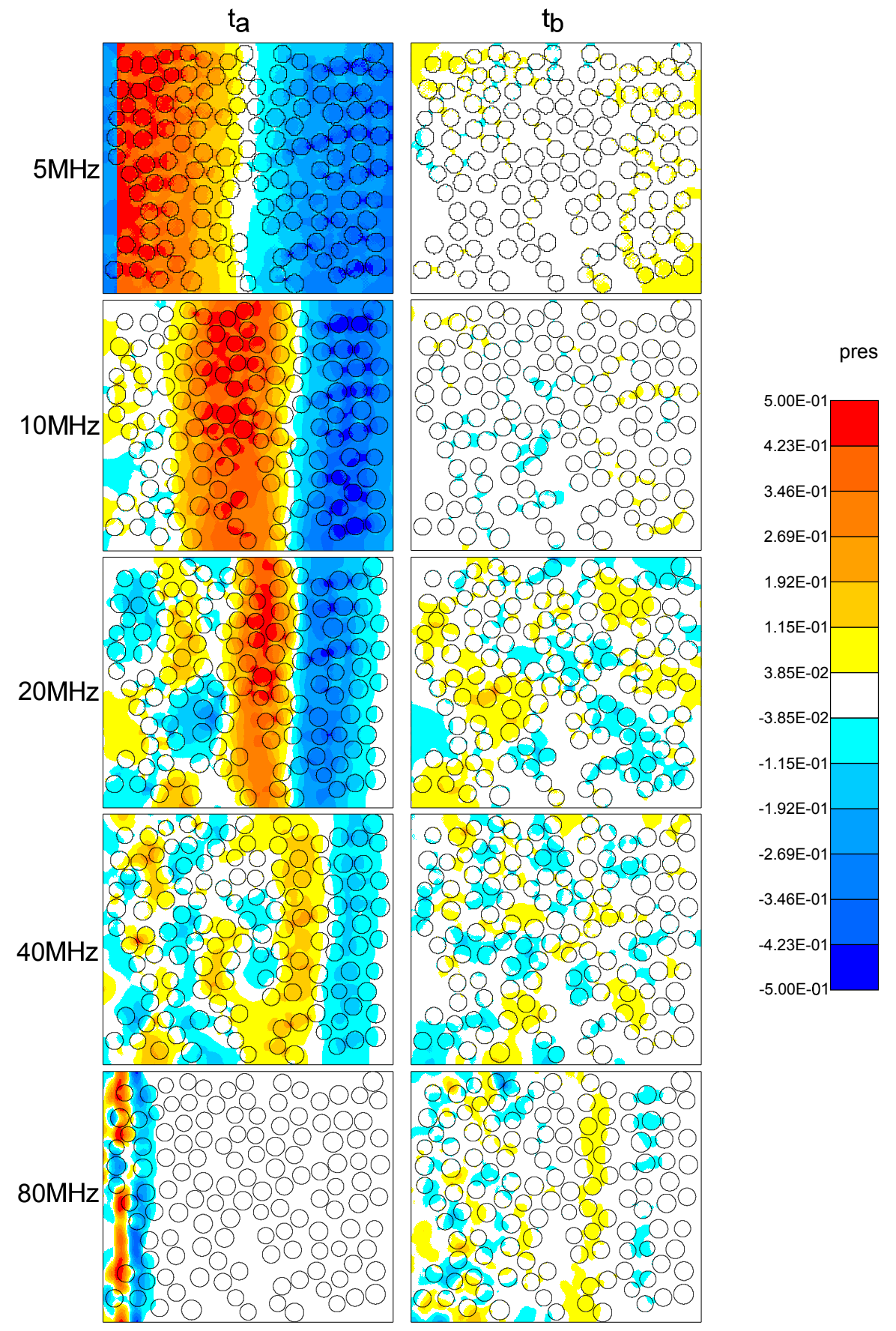

Figure 5.16: FEA snapshots of FRC rod cross section displaying the attenuating affects of scattering on a pulse of varied frequency. 

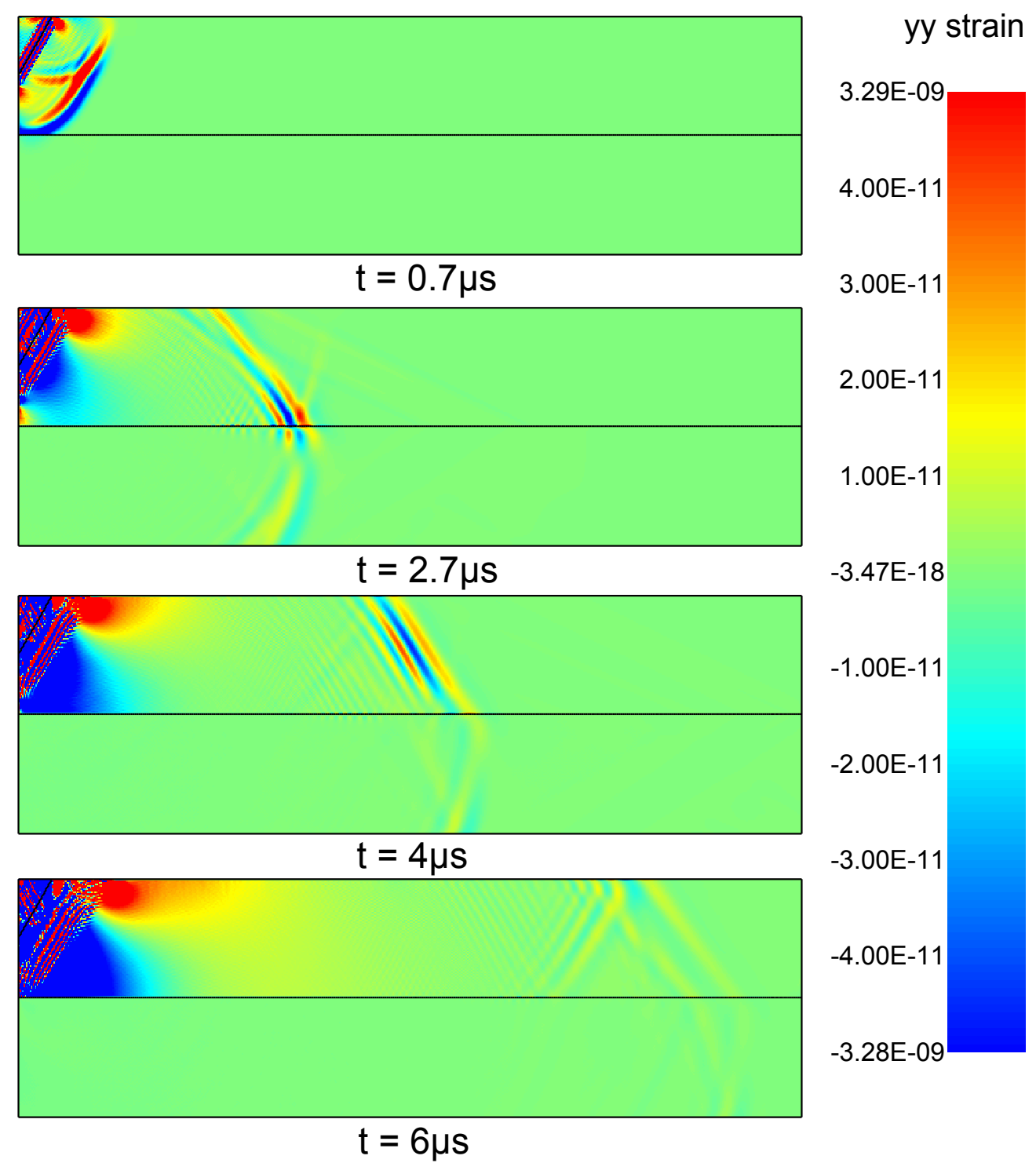

Figure 5.17: Snapshots of leaky Rayleigh wave propagating along the surface of a solid with density and velocity taken from measurements of a FRC sample. The leaky Rayleigh wave does not travel far before leaking it's energy due to the small impedance difference between the solid and water. Residual errors begin to accumulate and dominate the model. 


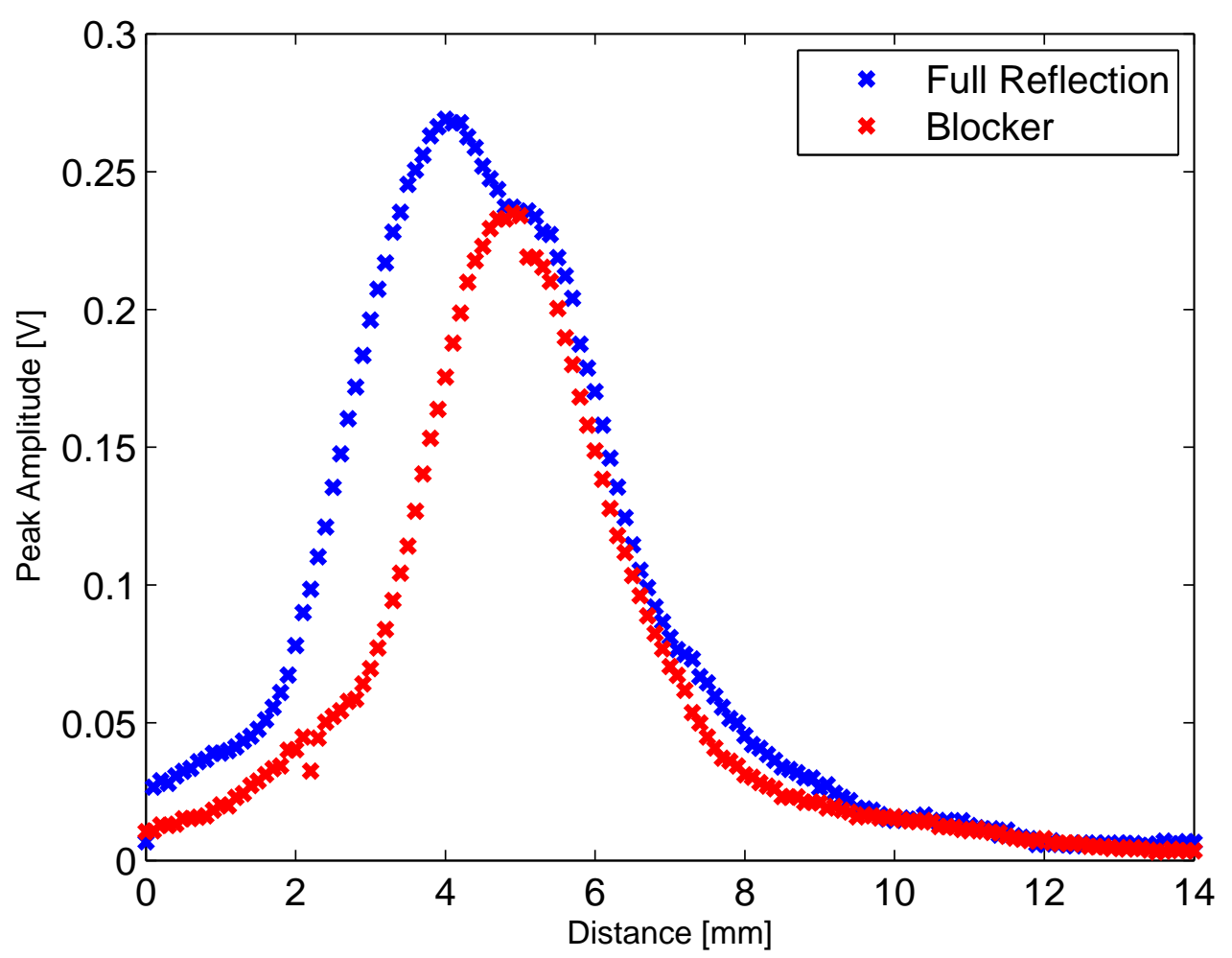

Figure 5.18: Amplitude of peak for each distance step extracted from measurements along the surface of FRC sample. The blue crosses represent the combination of the specular and non-specular reflections, the later identified by the second maxima. The red crosses are from the same measurement however the specular reflection was blocked by a microscope cover slip. 


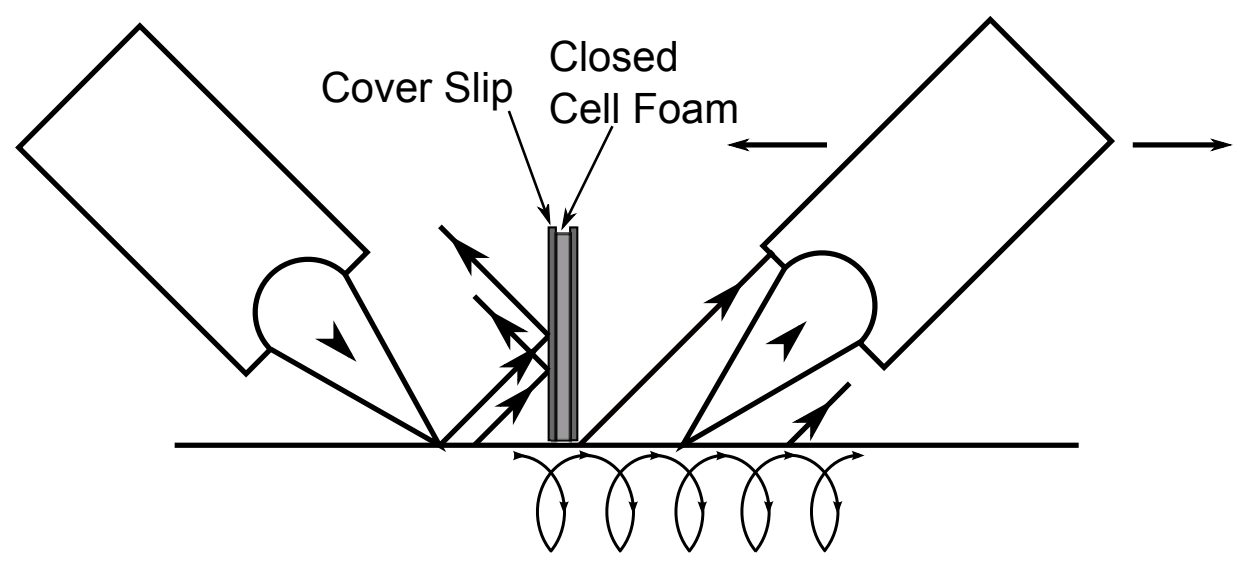

Figure 5.19: A specular reflection blocker comprising of closed cell foam sandwiched between two microscope cover slips.

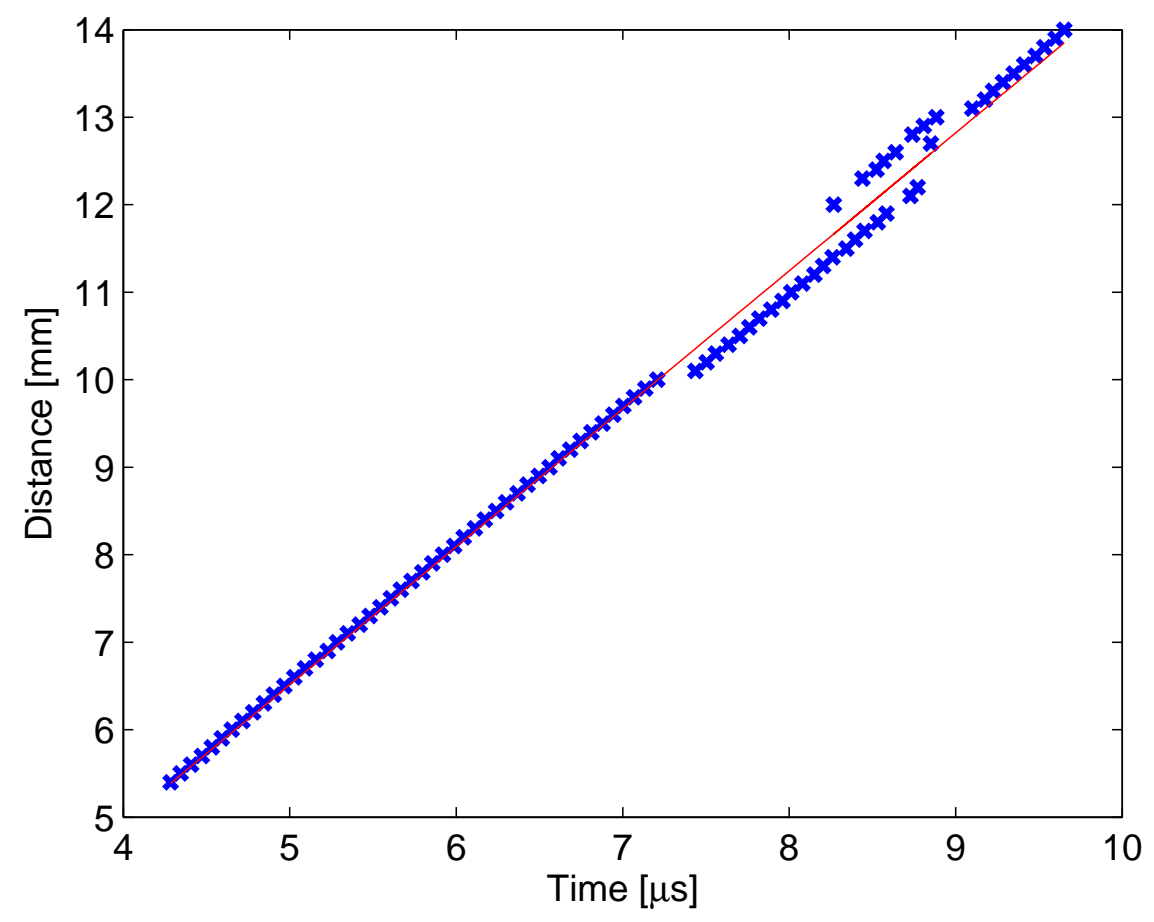

Figure 5.20: Time data for each peak in the velocity measurement plotted against distance. The slope of the fit was $1555 \mathrm{~ms}^{-1}$, faster than water but too low to be considered a leaky Rayleigh wave. 
The velocity was calculated as $1555 \mathrm{~ms}^{-1}$ with an excellent linear fit. This velocity was $75 \mathrm{~ms}^{-1}$ higher than water $\left(1480 \mathrm{~ms}^{-1}\right)$ corresponding to an error of $5 \%$. The calculated velocity was smaller than the expected Rayleigh velocity of $1640 \mathrm{~ms}^{-1}$, with an error of $5.2 \%$. With the same error it was impossible for this be considered a reliable measurement of velocity of a leaky Rayleigh wave. The measured velocity was confirmed to not be from a leaky Rayleigh wave from the phase measurement, where the phase of the wave received was not $\pi$ out of phase with the specular reflection. The source of the measured wave was believed to be from either diffraction off the base of the blocker or energy scattered off the fibres within the sample. The amplitude of the measured wave at $4.9 \mathrm{~mm}$ was comparable with that measured without the blocker. This would not have been the case if the measured wave was a Rayleigh wave since the leaky Rayleigh wave would have reduced in amplitude to less than $5 \%$ after travelling $1 \mathrm{~mm}$ under the blocker. The blocker was reduced to a single microscope slide but the blocking effect was found to be minimal.

The conclusion from this experiment was that the observed phenomenon was not a leaky Rayleigh wave, as the three criteria for a leaky Rayleigh wave (velocity, exponential amplitude decay and phase) were not met. This result was not unexpected given the small 1/e distance which meant the distance travelled by the leaky Rayleigh wave (ignoring absorption and scattering) would be less than $1 \mathrm{~mm}$ before the signal amplitude was significantly reduced.

A possible solution to the problem of the signal amplitude would be to increase the power of the pulsing signal. However the power amplifier available was designed to operate above $10 \mathrm{MHz}$ and the response at $5 \mathrm{MHz}$ was $60 \%$ of that at higher frequencies. The power of the acoustic pulse at such a low frequency was limited by the response of the $10 \mathrm{MHz}$ transducer, which had a very narrow bandwidth centered at $10 \mathrm{MHz}$ hence the 
response at $5 \mathrm{MHz}$ was weak. This issue of transducer response was compounded by the use of the $20 \mathrm{MHz}$ transducer as a receiver, which was centered at $29 \mathrm{MHz}$, with an even weaker response at $5 \mathrm{MHz}$.

The use of lower frequencies would result in an increase in the characteristic distance of the leaky Rayleigh wave. At $1 \mathrm{MHz}$ the $1 / e$ distance would increase by a factor of 5 . Lower frequencies would also lessen the extent to which the Rayleigh wave is scattered and absorped in the FRC. However the ability to detect shallow surface cracks may become compromised.

Irrespective of the chosen frequency the most significant factor in terms of increasing characteristic distance is the fluid. Water has a density/velocity ratio only half that of the FRC, resulting in a $95 \%$ amplitude reduction distance of approximately 6 wavelengths. If the fluid was changed to Isopropyl alcohol, the characteristic distance would increase to 9 wavelengths [11], a small difference. An order of magnitude (or two) increase in this characteristic distance is desired in order to propagate a Rayleigh wave around the full circumference of a rod. This was verified in an FEA model by reducing the density of the water by a factor of 100 , seen in Fig. 5.21. The leaky Rayleigh wave's amplitude was much greater after travelling the same distance which verified that a change of fluid would increase the $1 / e$ distance. 

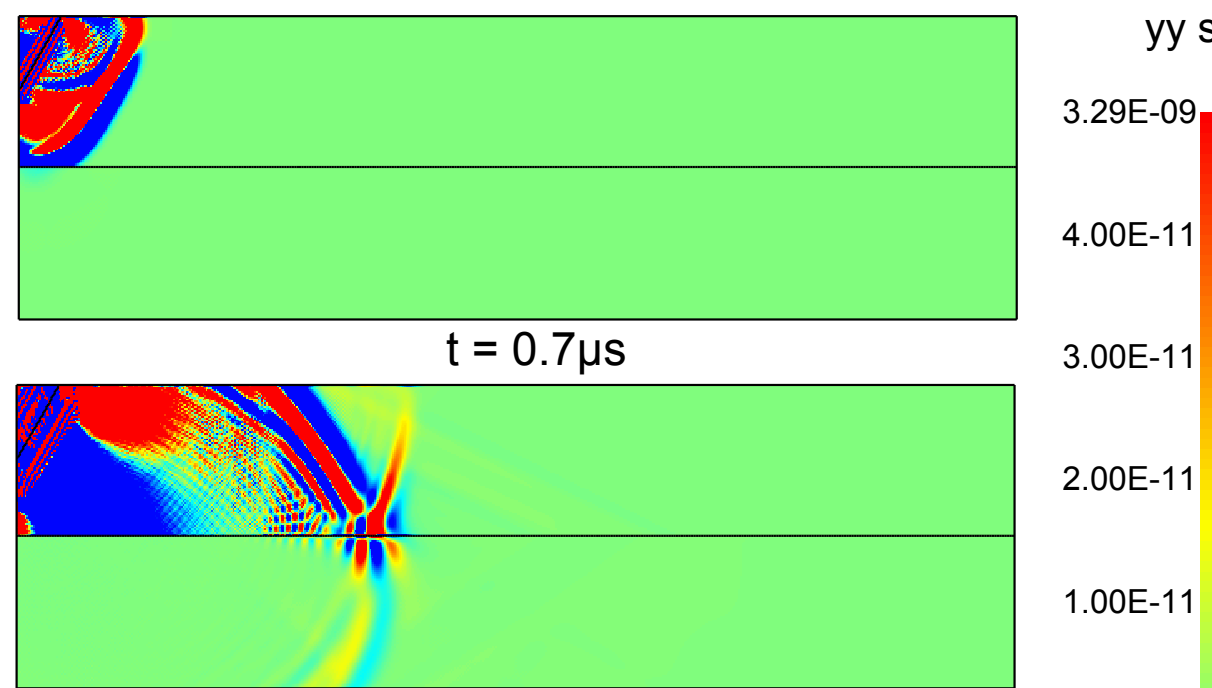

$\mathrm{t}=2.7 \mu \mathrm{s}$

$-3.47 E-18$

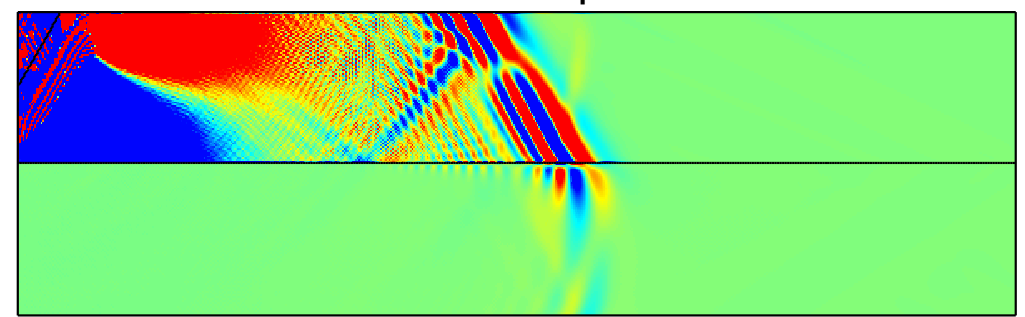

$\mathrm{t}=4 \mu \mathrm{s}$

2.00E-11

1.00E-11

$-1.00 \mathrm{E}-11$

$-2.00 \mathrm{E}-11$

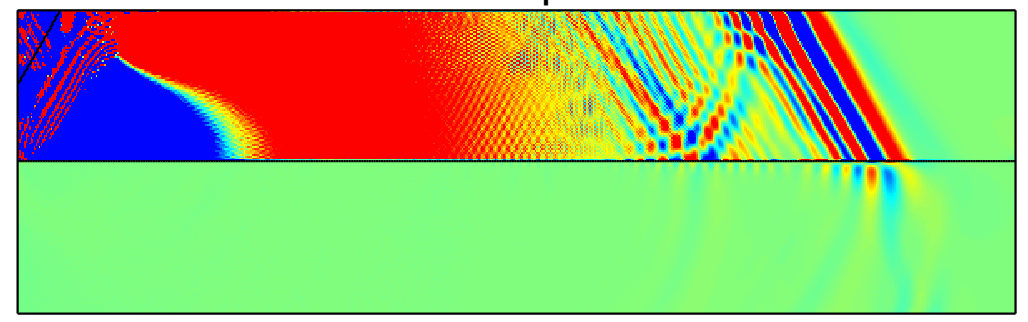

$-3.00 \mathrm{E}-11$

$-4.00 \mathrm{E}-11$

$-3.28 \mathrm{E}-09$

$$
t=6 \mu s
$$

Figure 5.21: FEA simulation of leaky Rayleigh wave propagating along the surface of the FRC. The water density was reduced by a factor of 100 to demonstrate the dependence of the characteristic distance on the impedance difference. The intensity of the leaky Rayleigh wave is significantly higher than that in Fig. 5.17. The effect of the residual error is more pronounced also, leading to a swamping of the simulation. 
The only known fluid that could provide the necessary impedance is actually a gas, such as air, where the characteristic distance would increase dramatically to approximately 8000 wavelengths. The attenuation in air above $1 \mathrm{MHz}$ is quite high, hence air coupled transducers are often replaced by lasers to excite ultrasound [9]. Detection of the Rayleigh wave could then be achieved via either a laser interferometer measuring the surface displacement, or air coupled transducers to detect the leaked energy. Alternatively a contact method for launching and detecting Rayleigh waves could involve a double wedge (acrylic/steel) setup [10] which does not require a coupling gel or immersion as the steel mediator is in direct contact with the sample.

\subsection{Conclusion}

This work provided an insight into the generation of leaky Rayleigh waves in glass and fibre reinforced composites. The generation of Rayleigh waves was achieved via critical angle excitation whilst detection involved measuring the energy leaked by the Rayleigh wave into water. The glass sample proved the efficacy of two methods for crack dection, pulse-echo and pitch-catch. The distances over which these methods would be reliable was limited to approximately $2 \mathrm{~mm}$, predominantly due to the small difference between the densities of the water relative to glass. The small amplitude of the Rayleigh wave could also be attributed to the scattering from the fibres acting as sub wavelength scatterers hence the affect of frequency was investigated with FEA simulations. A change of the fluid, i.e. water to air, would enable the Rayleigh wave to propagate further along the surface before leaking its energy. The same behaviour was observed for a fibre reinforced composite sample however the density difference was smaller resulting in the Rayleigh wave leaking its energy very fast, so much so that it couldn't be identified separately from the specular reflection. This lead to the conclusion that leaky Rayleigh waves in water were not an appro- 
priate tool for detecting surface cracks on the surface of fibre reinforced composites. Other methods for generating and detecting Rayleigh waves on a the surface of a fibre reinforced rod were introduced, particularly lasers and double wedge methods, however these were not implimented due to the availability of equipment and time restrictions on the project. 


\section{Chapter 6}

\section{Summary and Conclusion}

The motivation for this work was to develop high frequency $(>20 \mathrm{MHz})$ ultrasonic non-destructive techniques for detecting manufacturing flaws in fibre reinforced composites with particular focus on coating thickness and surface crack detection. The work on coating thickness measurements was successful as the thicknesses of a plastic sheath $(1 \mathrm{~mm})$ and a thin $(90 \mu \mathrm{m})$ layer of paint were measured accurately. FEA modelling demonstrated the effectiveness of a pulse-echo measurement at measuring thickness of a layer ultilising the time of flight data and a known velocity. Chirped waveforms and matched filtering were shown to improve the resolution limit of the system such that it was capable of measuring thickness greater than $20 \mu \mathrm{m}$. High frequency immersion transducers at a centre frequency of $50 \mathrm{MHz}$ were used to experimentally measure coating thicknesses with excellent results.

The work on crack detection provided an insight into the generation of leaky Rayleigh waves in glass and fibre reinforced composites using FEA. The generation of Rayleigh waves was achieved via critical angle excitation in water. Experimentally a glass sample proved the efficacy of two methods for crack dection, pulse-echo and pitch-catch. The distances over which these methods would be reliable was limited to approximately $3 \mathrm{~mm}$ 
predominantly due to the small difference in the acoustic properties of the water and glass. The methods were then applied to an FRC sample however the amplitude of the leaky Rayleigh wave decayed too quickly to be detected. This decay was attributed to the scattering from the fibres acting as sub wavelength scatterers and the small difference in acoustic properties between the water and FRC. FEA simulations demonstrated how the frequency and scatterer dimension were related and confirmed that a low excitation frequency was required in order to reduce scattering. To enable the leaky Rayleigh wave to travel further, a change of the fluid would be required i.e. water to air. This lead to the conclusion that leaky Rayleigh waves in water were not an appropriate tool for detecting surface cracks on the surface of fibre reinforced composites. Future work could involve other methods for generating and detecting Rayleigh waves on the surface of a fibre reinforced rod. Double wedge methods in air could enable a Rayleigh wave to propagate a large enough distance to be useful for crack detection. Laser ultrasonics are popular as they are non-contact, do not require a coupling medium and are accurate. 


\section{References}

[1] Olympus, Panametrics - Ultrasonic Transducers - Wedges, Cabes, Test Blocks. [Online; accessed 6-April-2011 http: //www.olympus-ims.com/data/File/panametrics/ panametrics-UT.en.pdf].

[2] A. Dawson, High Frequency Ultrasonic Wave Propagation In Anisotropic Materials. PhD thesis, Victoria University of Wellington, 2011.

[3] L. C. Hollaway, Handbook of Polymer Composites for Engineers. Woodhead Publishing Limited, 1994.

[4] Pultron Composites Ltd Website. Pultron Composites Ltd, 2011. [Accessed Online 11 June 2011 http: / / www . pultron . com].

[5] Private Communication. Pultron Composites Ltd, 2009.

[6] Y. Angel and J. D. Achenbach, "Reflection and transmission of obliquely incident Rayleigh waves by a surface-breaking crack," The Journal of the Acoustical Society of America, vol. 75, no. 2, p. 313, 1984.

[7] B. Masserey and E. Mazza, "Ultrasonic sizing of short surface cracks," Ultrasonics, vol. 46, no. 3, pp. 195-204, 2007.

[8] S.-H. Kee and J. Zhu, "Surface wave transmission measurements across distributed surface-breaking cracks using air-coupled sensors," Journal of Sound and Vibration, vol. 330, no. 22, pp. 5333 - 5344, 2011. 
[9] J. Littles Jr., L. Jacobs, and A.-H. Zureick, "Single-sided ultrasonic technique to characterize thick FRP composites," Journal of Nondestructive Evaluation, vol. 17, no. 4, pp. 223-230, 1998.

[10] B. Zurn and S. Mantell, "Nondestructive evaluation of laminated composites using Rayleigh waves," Journal of Composite Materials, vol. 35, no. 12, pp. 1026-1044, 2001.

[11] J. D. N. Cheeke, Fundamentals and Applications of Ultrasonic Waves. CRC Press, 2002.

[12] W. A. Inc, Weidlinger Associates Inc Website. Weidlinger Associates Inc, 2011. [Accessed Online 10 October 2011 http: / / www . pzf lex . com].

[13] L. E. Kinsler, A. R. Frey, A. B. Coppens, and J. V. Sanders, Fundamentals of Acoustics. Wiley, 1999.

[14] I. A. Viktorov, Rayleigh and Lamb Waves: Physical Theory and Applications. Plenum Press, New York, 1967.

[15] J. L. Rose, Ultrasonic Waves in Solid Media. Cambridge University Press, 2004.

[16] R. S. C. Cobbold, Foundations of Biomedical Ultrasound. Oxford University Press, 2007.

[17] T. L. Szabo, Diagnostic Ultrasonic Imaging: Inside Out. Elsevier, 2004.

[18] L. Rayleigh, "On waves propagated along the plane surface of an elastic solid," Proceedings of the London Mathematical Society, vol. s117 , no. 1, pp. 4-11, 1885.

[19] G. S. Kino, Acoustic Waves: Devices, Imaging and Analog Signal Processing. Prentice Hall, 1987. 
[20] E. W. M., W. S. Ardetzky, and F. Press, Elastic Waves in Layered Media (International Series in Earth Science). McGraw-Hill Education, 1957.

[21] allshookup.org. 2011.

[22] N. Declercq and E. Lamkanfi, "Study by means of liquid side acoustic barrier of the influence of leaky Rayleigh waves on bounded beam reflection," Applied Physics Letters, vol. 93, no. 5, 2008.

[23] Olympus, V390 Transducer Specification Sheet, 2006.

[24] Olympus, V372 Transducer Specification Sheet, 2011.

[25] J. W. S. L. Rayleigh), "On the light from the sky, its polarisation and colour," Philosophical Magazine, vol. 41, p. 107, 1871.

[26] Olympus, V327 Transducer Specification Sheet, 2011.

[27] "E1065 - standard guide for evaluating characteristics of ultrasonic search units," tech. rep., American Society for Testing and Materials, 2003.

[28] “Olympus - private communication." Email, September 2011.

[29] F. Harris, "On the use of windows for harmonic analysis with the discrete Fourier transform," Proceedings of the IEEE, vol. 66, pp. 51 83, January 1978.

[30] D. K. Hsu and M. S. Hughes, "Simultaneous ultrasonic velocity and sample thickness measurement and application in composites," The Journal of the Acoustical Society of America, vol. 92, no. 2, pp. 669-675, 1992.

[31] Minicircuits, ZHL-20W-13 Specification Sheet. [Online; accessed 6-May-2011 http://www.minicircuits.com/pdfs/ ZHL-20W-13+.pdf]. 
[32] J. Camacho and C. Fritsch, "Protection circuits for ultrasound applications," IEEE Transactions on Ultrasonics, Ferroelectrics, and Frequency Control, vol. 55, no. 5, pp. 1160-1164, 2008.

[33] Minicircuits, Gali74+ Monolithic Amplifier Specification Sheet. [Online; accessed 6-May-2011 http://www.minicircuits.com/pdfs/ GALI $-74+. p d f]$.

[34] Minicircuits, MVA-2000+ Voltage Variable Attenuator Specification Sheet. [Online; accessed 6-May-2011 http: / /www.minicircuits.com/ pdfs /MVA-2000+.pdf].

[35] A. Dawson, P. Harris, and G. Gouws, "Design and evaluation of ultrasonic transducer circuits for material characterisation," in Proceedings of the 13th Electronics New Zealand Conference, pp. 261-265, November 2006.

[36] A. P. Dawson, P. Harris, and G. J. Gouws, "High frequency ultrasound system for analysis of anisotropic materials," in Proceedings of the Fourteenth Electronics New Zealand Conference, pp. 137 - 140, November 2007.

[37] A. Dawson, P. Harris, and G. Gouws, "High frequency ultrasonic wave propagation in porous aluminium," in Smart Sensors and Sensing Technology (S. C. Mukhopadhyay and G. S. Gupta, eds.), vol. 20 of Lecture Notes in Electrical Engineering, pp. 221-232, Springer Berlin Heidelberg, 2008.

[38] B. R. Mahafza and A. Z. Elsherbeni, Matlab Simulations for Radar Systems Design. Chapman \& Hall/CRC Press, 2004.

[39] D. Morgan, Surface Acoustic Wave Filters (Studies in Electrical and Electronic Engineering). Academic Press, 2007. 
[40] T. Gan, D. Hutchins, R. Green, M. Andrews, and P. Harris, “Noncontact, high-resolution ultrasonic imaging of wood samples using coded chirp waveforms," IEEE Transactions on Ultrasonics, Ferroelectrics, and Frequency Control, vol. 52, no. 2, pp. 280-287, 2005.

[41] Weidlinger Associates Inc, PZFlex Command Reference, 2.4 ed., 2010.

[42] S.-H. Kee and J. Zhu, "Using air-coupled sensors to determine the depth of a surface-breaking crack in concrete," Journal of the Acoustical Society of America, vol. 127, no. 3, pp. 1279-1287, 2010.

[43] R. Kriz and W. Stinchcomb, "Elastic moduli of transversely isotropic graphite fibers and their composites," Experimental Mechanics, vol. 19, pp. 41-49, 1979.

[44] T. Peng, "Detect circles with various radii in grayscale image via Hough transform." [Accessed Online 13 June 2011 http: //www . mathworks.com/matlabcentral/fileexchange/9168].

[45] E. Lamkanfi, N. Declercq, W. Van Paepegem, and J. Degrieck, "Numerical study of Rayleigh wave transmission through an acoustic barrier," Journal of Applied Physics, vol. 105, no. 11, 2009. 